\title{
On the topological contents of $\eta$-invariants
}

\author{
Ulrich Bunke* \\ September 24, 2018
}

\begin{abstract}
We discuss an universal bordism invariant obtained from the Atiyah-PatodiSinger $\eta$-invariant from the analytic and homotopy theoretic point of view. Classical invariants like the Adams $e$-invariant, $\rho$-invariants and String-bordism invariants are derived as special cases. The main results are a secondary index theorem about the coincidence of the analytic and topological constructions and intrinsic expressions for the bordism invariants.
\end{abstract}

\section{Contents}

1 Introduction $\quad 2$

2 The topological construction 4

2.1 Motivation ........................ 4

2.2 The definition of $\eta^{t o p} \ldots \ldots \ldots \ldots \ldots$

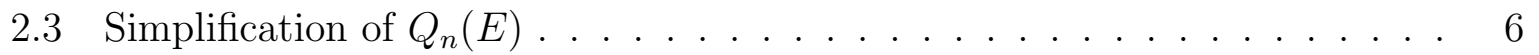

2.4 Relation with the Adams spectral sequence and $K$-localisation . . . . . . . 9

3 The spectral geometric construction 11

3.1 Motivation .......................... 11

3.2 Geometric cycles for $B$-bordism theory . . . . . . . . . . . . . . . 12

3.3 Normal and tangential Spin ${ }^{c}$-structures . . . . . . . . . . . . . . . . . . 13

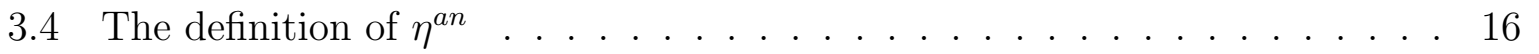

3.5 The secondary index theorem ................. 21

4 An intrinsic formula 31

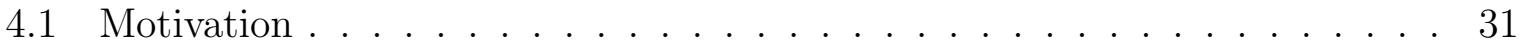

4.2 Review of differential $K$-theory . . . . . . . . . . . . . . . . . 32

4.3 Geometrisations ...................... 34

4.4 Good geometrisations . . . . . . . . . . . . . . . . . 40

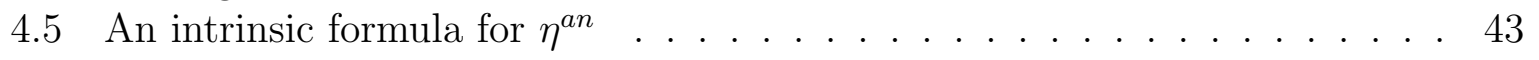

${ }^{*}$ NWF I - Mathematik, Universität Regensburg, 93040 Regensburg, GERMANY, ulrich.bunke@mathematik.uni-regensburg.de 
5 Examples 46

5.1 Adams' $e$-invariant . . . . . . . . . . . . . . . . 46

$5.2 \rho$-invariants and the index theorem for flat bundles $\ldots \ldots \ldots \ldots$

5.3 Algebraic $K$-theory . . . . . . . . . . . . . . . . . . . 50

5.4 String-bordism . . . . . . . . . . . . . . . . . . 54

5.5 The Crowley-Goette invariants . . . . . . . . . . . . . . . . 68

6 The $K$-theory of $K(\pi, n)$-bundles 77

\section{Introduction}

The purpose of this work is to understand which topological information is encoded in the $\eta$-invariant, a spectral-geometric invariant introduced by Atiyah-Patodi-Singer [5] in the context of index theory for boundary value problems for Dirac-operators. We are in particular interested in bordism invariants derived from the $\eta$ invariant. By now we know many examples, see e.g. [7],[11], [10], [21], [26], [39], [24]. In the present paper we consider the universal structure behind these examples. We define a bordism invariant which we call the universal $\eta$-invariant. We use Section 5 in order to review some of the known $\eta$-invariant based bordism invariants. We put the emphasis on the demonstration how they can be interpreted as special cases of our universal construction. Our construction also subsumes (by constructions similar to the one in Subsection 5.5) some, but not all, of the Kreck-Stolz type or Eells-Kuiper type invariants, [42, [27, or the generalised Rochlin invariants of [45]. The universal $\eta$-invariant does not seem to incorporate the invariant introduced by Kervaire-Milnor in order to distinguish exotic spheres [41].

In the present paper we introduce and compare two versions of the universal $\eta$-invariant. The analytic version $\eta^{a n}$ given in Definition 3.5 is the bordism invariant which is derived from the appearance of the reduced $\eta$-invariant in the local index theorem for the AtiyahPatodi-Singer boundary value problem by cancelling out the dependence on geometric data. The ideas for this construction are more or less standard and have been used previously in many special situations.

The topological counterpart $\eta^{t o p}$ introduced in Definition 2.3 is constructed by a simple homotopy-theoretic consideration using the interplay of $\mathbb{Q} / \mathbb{Z}$-bordism and $K$-theory. While it is not so complicated to see that $\eta^{a n}$ is a bordism invariant, to understand its homotopy-theoretic meaning is slightly deeper. The bridge between analysis and topology is provided by our first main Theorem 3.6 stating that

$$
\eta^{a n}=\eta^{t o p} .
$$

Its proof uses standard methods in index theory like the analytic picture of $K$-homology [13], [40], the Atiyah-Patodi-Singer index theorem [5], and some ideas from $\mathbb{Z} / l \mathbb{Z}$-index theory [30].

Bordism classes can be represented geometrically by manifolds with additional structures, called cycles (see Subsection 3.2 for details). It is then an interesting question how one 
can calculate the universal $\eta$-invariant or its specialisations in terms of the cycle. The definition of both, the topological or the analytical version of the universal $\eta$-invariant involves the choice of a zero bordism of some multiple of the cycle. In applications it is often complicated to find such a zero bordism. It is a striking advantage of the analytic picture that it can be reorganised to an expression which only involves structures on the cycle itself. In special cases this has been previously exploited in [50], 26] (the case of the Adams e-invariant, see Subsection 5.1), and [21] (to calculate String-bordism invariants, see Subsection 5.4).

We consider the intrinsic formula for $\eta^{a n}$ given in Theorem 4.19 as one of the main original contributions of the present paper. This formula is based on a new object which we call a geometrisation. If a map $f: M \rightarrow B G$ classifies a principal $G$-bundle $P$, then a geometrisation of $f$ is essentially given by a connection on $P$. In general, the notion of a geometrisation partially generalises the notion of a connection on the in general nonexistent principal bundle classified by the map $f: M \rightarrow B$ for an arbitrary space $B$. The details are slightly more complicated since we will take structures on the normal bundle into account.

Remark 1.1 In this paper we generally decided to work with complex $K$-theory. We think that there is a real version of the whole theory which can be obtained by replacing complex $K$-theory by real $K O$-theory, $B \operatorname{Spin}^{c}$ by BSpin, and taking the real structures on the spinor bundles into account properly on the analytic side. The real version of the universal $\eta$-invariant would be slightly stronger than its complex counterpart which loses some two-torsion classes. In order to recover the Adams e-invariant or the string bordism invariant [21] completely as special cases of the universal $\eta$-invariant we would need the real version.

Let us now describe the contents of the paper. In Section 2 we introduce the topological version $\eta^{\text {top }}$ of the universal $\eta$-invariant and study its properties. Most interesting is probably the relation with the Adams spectral sequence Proposition 2.7 which asserts that the universal $\eta$-invariant detects the first non-trivial sub-quotient of the bordism theory with respect to the $K$-theory based Adams filtration.

In Section 3 we introduce the analytic version $\eta^{a n}$ of the universal $\eta$-invariant and prove the secondary index Theorem 3.6 stating that $\eta^{a n}=\eta^{\text {top }}$. Before we can define $\eta^{a n}$ we have to recall in Subsections 3.2 and 3.3 some preliminary technical details concerning the relation of structures on the stable normal bundle as they come out of the Pontrjagin-Thom construction, and structures on the tangent bundle which will be used to do geometry and analysis.

Section 4 is devoted to geometrizations (Definition 4.5) and the intrinsic formula for $\eta^{a n}$ (Theorem 4.19).

In the last Section 5 we discuss in detail various specialisations of the universal $\eta$-invariant. It contains mainly a review of known constructions and results with slight improvements or generalisations at some points (e.g. Corollary 5.12). In the Propositions 5.13 and 5.14 we show how the usual geometric structures of Spin- and String-geometry (see [53] for the latter) give rise to geometrisations which lead to the known intrinsic formulas for 
the corresponding bordism invariants. It has been the initial motivation for this work to understand the general principles behind the String-bordism invariants introduced in [21]. It should be easy to adapt the arguments used here for the String $=M O\langle 8\rangle$-bordism case to bordism theories $M O\langle n\rangle$ associated to higher connected covers $B O\langle n\rangle$ of $B O$.

Acknowledgement: I thank Bernd Ammann, Sebastian Goette, Diarmuid Crowley, Niko Naumann for stimulating discussions. I am in particular grateful to $M$. Völkl for suggesting various inprovements. The pictures have been typeset using the frobeniusgraphcalc. stypackage written by Clara Löh.

\section{The topological construction}

\subsection{Motivation}

In this section we use complex $K$-theory in order to detect torsion elements in the homotopy groups $\pi_{*}(E)$ of a spectrum $E$. We introduce the topological version $\eta^{\text {top }}$ of the universal $\eta$-invariant as a secondary version of the degree $\epsilon: \pi_{*}(E) \rightarrow \pi_{*}(K \wedge E)$. The idea is to first lift the torsion element to the $\mathbb{Q} / \mathbb{Z}$-version of $E$, then apply the degree, and finally to detect the result via its evaluations against $K^{0}(E)$. This construction is a generalisation of the construction of the Adams $e$-invariant in the case of the sphere spectrum $E=S$.

In Subsection 2.2 we will give the construction of the invariant. In Subsection 2.3 we analyse its target in some detail. Finally, in Subsection 2.4 we understand completely in terms of the Adams spectral sequence which piece of $\pi_{*}(E)_{\text {tors }}$ the universal $\eta$-invariant can detect.

\subsection{The definition of $\eta^{t o p}$}

For an abelian group $A$ we let $\mathrm{M} A$ denote the Moore spectrum which is characterised by its integral homology

$$
\pi_{n}(H \mathbb{Z} \wedge \mathrm{M} A) \cong\left\{\begin{array}{cc}
A & n=0 \\
0 & n \neq 0
\end{array} .\right.
$$

More generally, for a spectrum $E$ we have short exact sequences

$$
0 \rightarrow \pi_{n}(E) \otimes A \rightarrow \pi_{n}(E \wedge \mathrm{M} A) \rightarrow \pi_{n-1}(E) * A \rightarrow 0
$$

([17, (2.1)]) for all $n \in \mathbb{Z}$. In order to simplify the notation we abbreviate $E A:=E \wedge \mathrm{M} A$. The equivalences $\mathbf{M} \mathbb{Z} \cong S$ and $\mathbb{M} \cong H \mathbb{Q}$ induce equivalences $E \mathbb{Z} \cong E$ and $E \mathbb{Q} \cong E \wedge H \mathbb{Q}$. The starting point for the construction of the topological version $\eta^{\text {top }}$ of the universal $\eta$-invariant is the fibre sequence

$$
\mathrm{M} \mathbb{Z} \rightarrow \mathrm{MQ} \rightarrow \mathrm{MQ} / \mathbb{Z} \rightarrow \Sigma \mathrm{M} \mathbb{Z}
$$


of Moore spectra. Smashing this sequence with the map $\epsilon: E \rightarrow K \wedge E$ induced by the unit of the $K$-theory spectrum we get the diagram

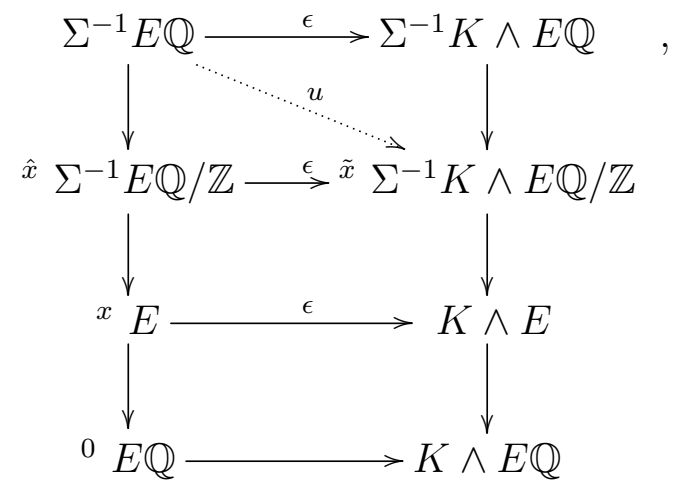

where we use the symbol $\epsilon$ also to denote other maps naturally induced by the unit.

Remark 2.1 If $E$ is a spectrum and $X$ is a space or spectrum, then for $n \in \mathbb{Z}$ we consider the cohomology $E^{n}(X)$ as a topological group where a basis of neighbourhoods of zero is given by the kernels of restrictions along maps $Y \rightarrow X$ from finite $C W$-complexes or finite cell spectra $Y$. The topology on $E^{n}(X)$ is called the profinite topology. This should not be confused with the notion of a profinite group in algebra.

We have an evaluation pairing

$$
\langle-,-\rangle: \pi_{n}\left(\Sigma^{-1} K \wedge E \mathbb{Q} / \mathbb{Z}\right) \otimes K^{0}(E) \rightarrow \pi_{n+1}(K \mathbb{Q} / \mathbb{Z})
$$

which sends $x \otimes \phi$ to the composition

$$
\Sigma^{n+1} S \stackrel{x}{\rightarrow} K \wedge E \wedge \mathrm{MQ} / \mathbb{Z} \stackrel{\mathrm{id}_{K} \wedge \phi \wedge \mathrm{id} \mathrm{MQ}_{\mathbb{Q}} \mathbb{Z}}{\longrightarrow} K \wedge K \wedge \mathrm{MQ} / \mathbb{Z} \stackrel{\mu}{\rightarrow} K \wedge \mathrm{MQ} / \mathbb{Z}
$$

where $\mu$ is the multiplication of the ring spectrum $K$. Its adjoint is a map

$$
\pi_{n}\left(\Sigma^{-1} K \wedge E \mathbb{Q} / \mathbb{Z}\right) \rightarrow \operatorname{Hom}^{\operatorname{cont}}\left(K^{0}(E), \pi_{n+1}(K \mathbb{Q} / \mathbb{Z})\right)
$$

where $\operatorname{Hom}^{\text {cont }}(-,-)$ stands for continuous homomorphisms and $\pi_{n+1}(K \mathbb{Q} / \mathbb{Z})$ has the discrete topology. We let

$$
U \subseteq \operatorname{Hom}^{\text {cont }}\left(K^{0}(E), \pi_{n+1}(K \mathbb{Q} / \mathbb{Z})\right)
$$

denote the subgroup given by the pairings with the elements in the image

$$
\text { image }\left(u: \pi_{n}\left(\Sigma^{-1} E \mathbb{Q}\right) \rightarrow \pi_{n}\left(\Sigma^{-1} K \wedge E \mathbb{Q} / \mathbb{Z}\right)\right)
$$

of the map $u$ which can be read off from (3).

Definition 2.2 For every $n \in \mathbb{Z}$ we define the abelian group

$$
Q_{n}(E):=\frac{\operatorname{Hom}^{c o n t}\left(K^{0}(E), \pi_{n+1}(K \mathbb{Q} / \mathbb{Z})\right)}{U}
$$


The following definition uses a diagram chase of elements as indicated in (3).

Definition 2.3 The homotopy theoretic version of the universal $\eta$-invariant is the homomorphism

$$
\eta^{\text {top }}: \pi_{n}(E)_{\text {tors }} \rightarrow Q_{n}(E)
$$

defined by the following prescription: If $x \in \pi_{n}(E)_{\text {tors }}$, then we choose a lift $\hat{x} \in \pi_{n}\left(\Sigma^{-1} E \mathbb{Q} / \mathbb{Z}\right)$.

We let $\eta^{\text {top }}(x) \in Q_{n}(E)$ be the class represented by the homomorphism given by the pairing against $\epsilon(\hat{x})$.

We must show that $\eta^{\text {top }}$ is well-defined. Indeed, the choice of $\hat{x}$ is unique up to elements which come from $\pi_{n}\left(\Sigma^{-1} E \mathbb{Q}\right)$, but this ambiguity is taken care of by taking the quotient by $U$ in the definition (6) of $Q_{n}(E)$.

The following Lemma immediately follow from the definitions:

Lemma 2.4 A map of spectra $E \rightarrow F$ naturally induces a commutative diagram

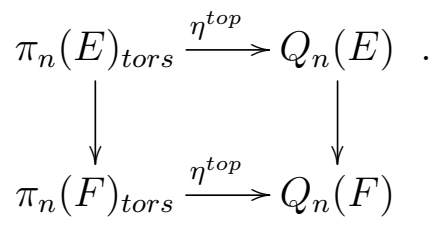

\subsection{Simplification of $Q_{n}(E)$}

Because of its definition as a quotient it is difficult to define maps out of $Q_{n}(E)$. In this subsection we analyze the structure of this group and explain how one can detect its elements. We consider the ring spectrum

$$
H P \mathbb{Q}:=H \mathbb{Q}\left[b, b^{-1}\right],
$$

where $\operatorname{deg}(b)=-2$. Additively it has a decomposition

$$
H P \mathbb{Q} \cong \bigvee_{i \in \mathbb{Z}} \Sigma^{2 i} H \mathbb{Q}
$$

and for each $i \in \mathbb{Z}$ we consider the projection $p_{2 i}: H P \mathbb{Q} \rightarrow \Sigma^{2 i} H \mathbb{Q}$ to the corresponding component. We set $p_{n}:=0$ for odd $n$. The Chern character is an equivalence of ring spectra

$$
\text { ch }: K \mathbb{Q} \stackrel{\sim}{\rightarrow} H P \mathbb{Q} .
$$

The composition of the Chern character with the projection $p_{n+1}$ gives a map

$$
p_{n+1} \circ \mathbf{c h}: K^{0}(E) \rightarrow H \mathbb{Q}^{n+1}(E)
$$

whose kernel will be denoted by

$$
V_{n}:=\operatorname{ker}\left(p_{n+1} \circ \mathbf{c h}\right)
$$


Lemma 2.5 1. The restriction to $V_{n} \subseteq K^{0}(E)$ induces a well-defined map

$$
Q_{n}(E) \rightarrow \operatorname{Hom}^{\text {cont }}\left(V_{n}, \pi_{n+1}(K \mathbb{Q} / \mathbb{Z})\right) .
$$

2. This restriction map is an isomorphism if we assume that $E$ is lower bounded and $\operatorname{dim} H \mathbb{Q}^{n+1}(E)$ is finite.

Proof. First we show that the restriction is well-defined. We must show that if $\phi \in V_{n}$, then the pairing of $\phi$ with $u(y) \in \pi_{n+1}(K \wedge E \mathbb{Q} / \mathbb{Z})$ vanishes for every $y \in \pi_{n+1}(E \mathbb{Q})$. This follows from the equality

$$
\langle u(y), \phi\rangle=q\left(\left\langle\operatorname{ch}(\epsilon(y)), p_{n+1}(\operatorname{ch}(\phi))\right\rangle\right)
$$

where

$$
q: \pi_{n+1}(H P \mathbb{Q}) \stackrel{\operatorname{ch}^{-1}}{\rightarrow} \pi_{n+1}(K \mathbb{Q}) \rightarrow \pi_{n+1}(K \mathbb{Q} / \mathbb{Z})
$$

and $\epsilon$ is as in (3).

We now show 2. We use the general fact that if $f: A \rightarrow V_{n}$ is a homomorphism of an abelian group into a $\mathbb{Q}$-vector space such that its image is finitely generated as an abelian group, then there exists a splitting $A \cong \operatorname{ker}(f) \oplus A^{\prime}$. Indeed, in this case the image is free and hence projective.

Note that there exists an integer $N$ (only depending on $n$ and the lower bound of $E$ ) such that the image of $p_{n+1} \circ \mathbf{c h}(\ldots)$ is contained in the image of $\frac{1}{N} H \mathbb{Z}^{n+1}(E) \rightarrow H \mathbb{Q}^{n+1}(E)$ and is therefore finitely generated as an abelian group since we assume that $H \mathbb{Q}^{n+1}(E)$ is finite dimensional. We conclude that

$$
K^{0}(E) \cong V_{n} \oplus V_{n}^{c},
$$

where $V_{n}^{c} \cong$ image $\left(p_{n+1} \circ \mathbf{c h}\right)$ is a free abelian group. This immediately implies that (9) is surjective.

Any homomorphism $\phi \in \operatorname{Hom}^{\text {cont }}\left(K^{0}(E), \pi_{n+1}(K \mathbb{Q} / \mathbb{Z})\right)$ can uniquely be decomposed as a sum of its restrictions to $V_{n}$ and $V_{n}^{c}$. We claim that $U \cong \operatorname{Hom}\left(V_{n}^{c}, \pi_{n+1}(K \mathbb{Q} / \mathbb{Z})\right)$, where $U$ is as in (5). The claim implies that (9) is injective.

We can assume that $n$ is odd. The claim follows from the surjectivity of the following composition

$\pi_{n}\left(\Sigma^{-1} E \mathbb{Q}\right) \cong \operatorname{Hom}^{\text {cont }}\left(H \mathbb{Q}^{n+1}(E), \mathbb{Q}\right) \cong \operatorname{Hom}^{\text {cont }}\left(V_{n}^{c}, \pi_{n+1}(K \mathbb{Q})\right) \rightarrow \operatorname{Hom}^{\text {cont }}\left(V_{n}^{c}, \pi_{n+1}(K \mathbb{Q} / \mathbb{Z})\right)$,

where we use the Chern character for the second equivalence and the fact that $V_{n}^{c}$ is free in order to conclude the surjectivity of the last map. Note that in the present situation continuity of homomorphisms is automatic by our finiteness assumption.

The definition of $\eta^{\text {top }}$ is based on first lifting the torsion element in the homotopy group of $E$ to a $\mathbb{Q} / \mathbb{Z}$ - homotopy class which is then paired with elements of $K$-theory. The pairing with torsion $K$-theory elements can be expressed in a dual way as a pairing of the original homotopy class with $\mathbb{Q} / \mathbb{Z}$-lifts of the $K$-theory elements. We now explain the details. 
Assume that $\phi \in K^{0}(E)$ satisfies $\operatorname{ch}(\phi)=0$. Then $\phi \in V_{n}$ and we get an evaluation

$$
\mathrm{ev}_{\phi}: Q_{n}(E) \rightarrow \pi_{n+1}(K \mathbb{Q} / \mathbb{Z}) .
$$

In view of the exact sequence

$$
K \mathbb{Q} / \mathbb{Z}^{-1}(E) \stackrel{\partial}{\rightarrow} K^{0}(E) \stackrel{\text { ch }}{\rightarrow} H P \mathbb{Q}^{0}(E)
$$

we can choose $\hat{\phi} \in K \mathbb{Q} / \mathbb{Z}^{-1}(E)$ such that $\partial \hat{\phi}=\phi$. If we want to calculate ev ${ }_{\phi}\left(\eta^{\text {top }}(x)\right)$, then instead of lifting the class $x$ to a $\mathbb{Q} / \mathbb{Z}$ class we can instead evaluate the class $\epsilon(x) \in$ $\pi_{n}(K \wedge E)$ against the lift $\hat{\phi}$. The following assertion follows easily from the definition of $\eta^{\text {top }}$ and commutativity of the diagram

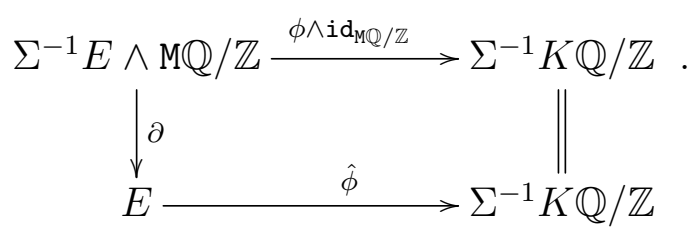

Lemma 2.6 For $x \in \pi_{n}(E)_{\text {tors }}$ and $\hat{\phi} \in K \mathbb{Q} / \mathbb{Z}^{-1}(E)$ we have

$$
\mathrm{ev}_{\phi}\left(\eta^{\text {top }}(x)\right)=\langle\hat{\phi}, \epsilon(x)\rangle \text {, }
$$

where $\phi:=\partial \hat{\phi}$.

In the present paper the spectrum $E$ will often be a Thom spectrum $M B$ associated to a map of spaces $B \rightarrow B \operatorname{Spin}^{c}$. The spectrum $M B$ is $K$-oriented by

$$
\beta: M B \rightarrow M \operatorname{Spin}^{c} \stackrel{A B S}{\longrightarrow} K,
$$

where $A B S$ is the Atiyah-Bott-Shapiro orientation. In this case can use the Thom isomorphisms

$$
\operatorname{Thom}^{K}: K^{0}(B) \stackrel{\sim}{\rightarrow} K^{0}(M B), \quad \operatorname{Thom}_{H \mathbb{Q}}: H \mathbb{Q}_{n+1}(M B) \stackrel{\sim}{\rightarrow} H \mathbb{Q}_{n+1}(B)
$$

in order to express $Q_{n}(M B)$ in terms of $B$. We have an isomorphism

$$
\left.Q_{n}(M B) \cong \frac{\operatorname{Hom}^{\text {cont }}\left(K^{0}(B), \pi_{n+1}(K \mathbb{Q} / \mathbb{Z})\right)}{U^{\prime}}\right),
$$

where $U^{\prime}$ is obtained by precomposing the elements in $U$ (see (5D) with $\operatorname{Thom}^{K}$. We let $\mathbf{T d} \in H P \mathbb{Q}^{0}\left(B \operatorname{Spin}^{c}\right)$ be the universal Todd class and use the same symbol in order to denote the pull-back of this class to $B$. Then using (10), the isomorphism

$$
\pi_{n+1}(M B \mathbb{Q}) \cong H \mathbb{Q}_{n+1}(M B) \stackrel{\text { Thom }_{H Q}}{\cong} H \mathbb{Q}_{n+1}(B),
$$

and the Riemann-Roch theorem we can describe $U^{\prime}$ as the space homomorphisms given by

$$
K^{0}(B) \ni \phi \mapsto q\left(\left\langle y, p_{n+1}\left(\mathbf{T d}^{-1} \cup \mathbf{c h}(\phi)\right)\right\rangle\right) \in \pi_{n+1}(K \mathbb{Q} / \mathbb{Z})
$$

for all $y \in H \mathbb{Q}_{n+1}(B)$. 


\subsection{Relation with the Adams spectral sequence and $K$-localisation}

In this subsection we will see that the topological $\eta$-invariant essentially detects the first line in the $K$-based Adams spectral sequence $\left(E_{r}^{*, *}(E), d_{r}\right)$ for $E$. We refer to [3] and [48] for details on the Adams spectral sequence. We define the spectrum $\bar{K}$ by the cofibre sequence

$$
\Sigma^{-1} \bar{K} \rightarrow S \rightarrow K \rightarrow \bar{K}
$$

and form the Adams tower

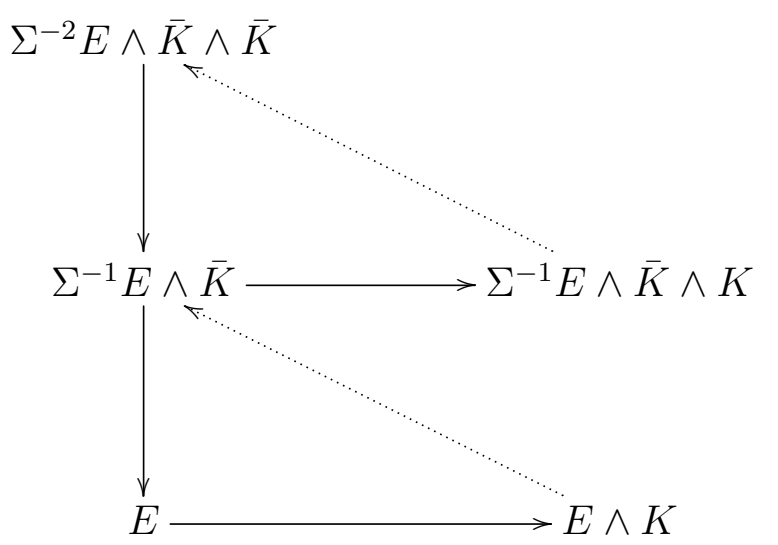

The horizontal maps are induced by the unit $S \rightarrow K$ while the dotted arrows are degree one-maps which turn the triangles into fibre sequences. This tower gives rise to an Adams spectral sequence

$$
\left(E_{r}^{*, *}(E), d_{r}\right)_{r \geq 1}
$$

and a filtration $\left(\mathcal{F}^{s} \pi_{*}(E)\right)_{s \geq 0}$ of the homotopy groups of $E$, where $\mathcal{F}^{s} \pi_{*}(E) \subseteq \pi_{*}(E)$ is defined as the image of $\pi_{*}\left(\Sigma^{-s} E \wedge \bar{K}^{\wedge s}\right)$. We have a natural injective map

$$
s: \operatorname{Gr}^{1} \pi_{*}(E) \rightarrow E_{2}^{1, *+1}(E) .
$$

Proposition 2.7 1. We have $\mathcal{F}^{1} \pi_{*}(E) \subseteq \pi_{*}(E)_{\text {tors }}$.

2. We have $\mathcal{F}^{2} \pi_{*}(E) \subseteq \operatorname{ker}\left(\eta^{\text {top }}\right)$. Hence $\eta^{\text {top }}$ induces a map (denoted by the same symbol)

$$
\eta^{t o p}: \operatorname{Gr}^{1} \pi_{*}(E) \rightarrow Q_{*}(E)
$$

3. There exists a map

$$
\kappa: E_{2}^{1, *+1}(E) \rightarrow Q_{*}(E)
$$

such that

$$
\eta^{t o p}=\kappa \circ s: \operatorname{Gr}^{1} \pi_{n}(E) \rightarrow Q_{n}(E)
$$

4. If $\pi_{*}(E \wedge K)$ is torsion-free, then

$$
\pi_{*}(E)_{\text {tors }}=\mathcal{F}^{1} \pi_{*}(E) .
$$


If $n$ is odd, then the restriction

$$
\kappa_{\mid E_{2}^{1, n+1}(E)_{t o r s}}: E_{2}^{1, n+1}(E)_{t o r s} \rightarrow Q_{n}(E)
$$

of $\kappa$ to the torsion subgroup is injective Consequently, the map

$$
\eta^{\text {top }}: \mathrm{Gr}^{1} \pi_{n}(E) \rightarrow Q_{n}(E)
$$

induced by $\eta^{\text {top }}$ is injective.

Proof. We have a map

$$
f: K \rightarrow K \mathbb{Q} \cong \prod_{p \in \mathbb{Z}} \Sigma^{2 p} H \mathbb{Q} \stackrel{\text { pr }}{\rightarrow} H \mathbb{Q}
$$

which we use to build the diagram

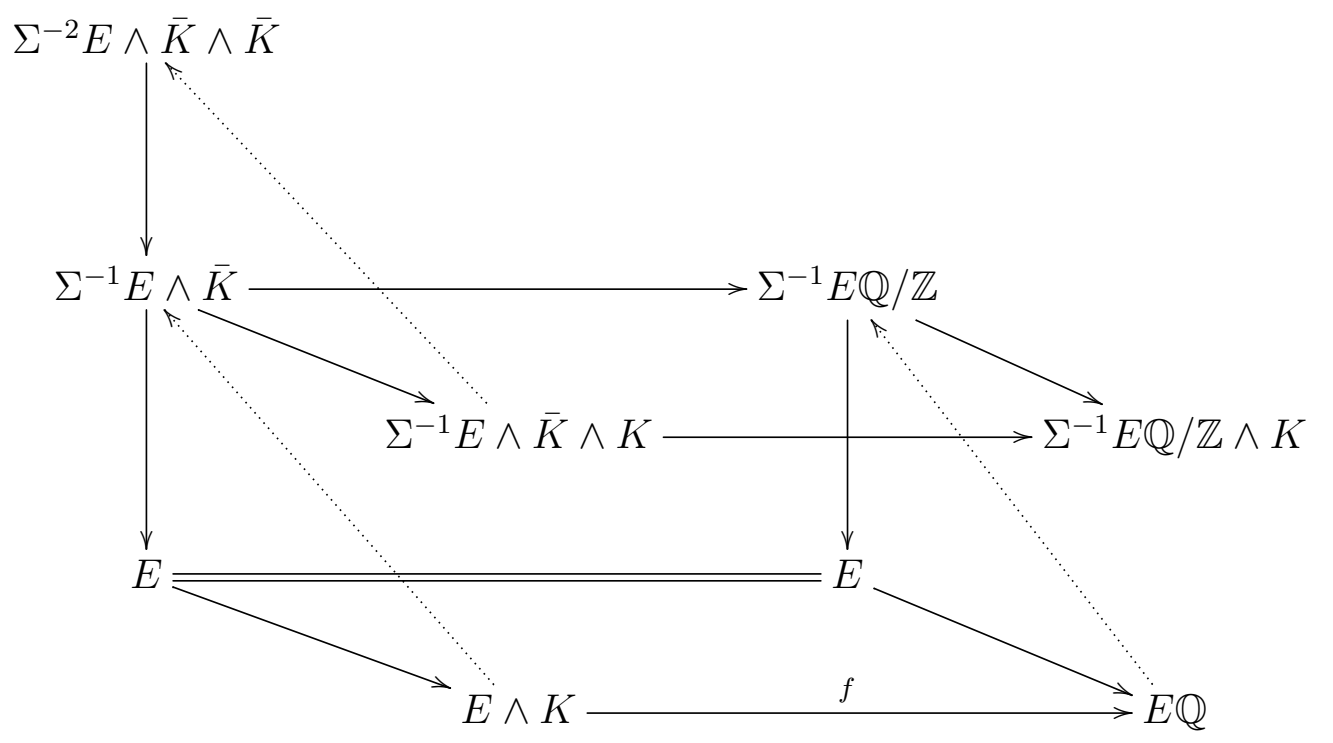

It connects a piece of the Adams tower of $E$ with the basic diagram used in the definition of $\eta^{\text {top }}$.

Assertions 1. and 2. follow immediately from a diagram chases. We now show assertion 3. The map

$$
E_{1}^{1, *+1}(E)=\pi_{*}\left(\Sigma^{-1} E \wedge \bar{K} \wedge K\right) \rightarrow \pi_{*}\left(\Sigma^{-1} E \mathbb{Q} / \mathbb{Z} \wedge K\right) \rightarrow Q_{*}(E)
$$

annihilates the image of $E \wedge K$, i.e the image of the boundary map of the spectral sequence and therefore factors through a map

$$
\kappa: E_{2}^{1, *+1}(E) \rightarrow Q_{n}(E) .
$$

Assertion 3. again follows by again by a diagram chase. 
We now show Assertion 4. In view of 1. the first part is clear. As in [21, Sec. 5.3] our additional assumption implies that we have a short exact sequence

$$
0 \rightarrow E_{2}^{0, n+1}(E) \otimes \mathbb{Q} / \mathbb{Z} \stackrel{j}{\rightarrow} E_{2}^{0, n+1}(E \mathbb{Q} / \mathbb{Z}) \stackrel{\delta}{\rightarrow} E_{2}^{1, n+1}(E)_{\text {tors }} \rightarrow 0
$$

Let $\gamma \in E_{2}^{1, n+1}(E)_{\text {tors }}$ and assume that $\kappa(\gamma)=0$. We write $\gamma=\delta(\beta)$ for some $\beta \in$ $E_{2}^{0, n+1}(E \mathbb{Q} / \mathbb{Z})$. We have $\kappa(\gamma)=0$ if an only if there exists $y \in \pi_{n+1}(E \mathbb{Q})$ which induces the same pairing with $K^{0}(E)$ as $a(\beta)$, where $a: E_{2}^{0, n+1}(E \mathbb{Q} / \mathbb{Z}) \rightarrow \pi_{n+1}(E \mathbb{Q} / \mathbb{Z} \wedge K)$ is the inclusion. By Pontrjagin duality we have

$$
\pi_{n+1}(E \mathbb{Q} / \mathbb{Z} \wedge K) \cong \operatorname{Hom}^{\text {cont }}\left(K^{n+1}(E), \mathbb{Q} / \mathbb{Z}\right) \cong \operatorname{Hom}^{\operatorname{cont}}\left(K^{0}(E), \pi_{n+1}(K \mathbb{Q} / \mathbb{Z})\right)
$$

We conclude that $a(\beta)=a(j(q(\epsilon(y))))$, where $\epsilon(y) \in E_{2}^{0, n+1}(E \mathbb{Q})$ and

$$
q: E_{2}^{0, n+1}(E \mathbb{Q}) \cong E_{2}^{0, n+1}(E) \otimes \mathbb{Q} \rightarrow E_{2}^{0, n+1}(E) \otimes \mathbb{Q} / \mathbb{Z} .
$$

Since $a$ is injective this implies $\gamma=\delta(j(q(\epsilon(y))))=0$.

We let $E \rightarrow E_{K}$ denote the Bousfield localisation of the spectrum $E$ at the complex $K$-theory spectrum. The following Lemma shows that the universal $\eta$-variant factorizes over the $K$-localisation.

Lemma 2.8 We have a commuting diagram

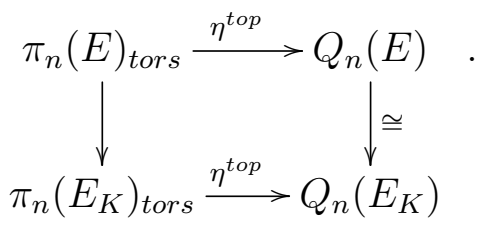

Proof. Since $E \rightarrow E_{K}$ induces an isomorphism in $K$-theory we conclude that the right vertical map is an equivalence. The rest is Lemma 2.4.

\section{The spectral geometric construction}

\subsection{Motivation}

In this section we define an analytic invariant $\eta^{a n}$ of torsion elements in the $B$-bordism theory. The analytic invariant will be derived from geometric and spectral geometric quantities associated to geometric cycles for bordism classes. The relation between the geometric and homotopy theoretic picture of the bordism group is given by Thom-Pontrjagin construction, see [49, Ch IV.7]. In Subsection 3.2 we give the details of the geometric picture of the $B$-bordism theory. Subsection 3.3 is devoted to some technical details on the transfer of $\operatorname{Spin}^{c}$-structures from the normal bundle to the tangent bundle. A reader with some experience with the Thom-Pontrjagin construction and Spin $^{c}$-structures may immediately proceed to the construction of $\eta^{a n}$ in Subsection 3.4. The final Subsection 3.5 of this part contains the proof of the theorem about the equality of the analytic and topological universal $\eta$-invariant. 


\subsection{Geometric cycles for $B$-bordism theory}

We assume that we have chosen representatives of the homotopy types of the classifying spaces of the classical Lie groups like $O(k)$, $\operatorname{Spin}^{c}(k)$ etc. and representatives of the homotopy classes of the usual maps connecting them like $B \operatorname{Spin}^{c}(k) \rightarrow B O(k)$ or $\iota_{k}$ : $B O(k) \rightarrow B O(k+1)$. We further choose universal euclidean bundles $\xi_{k}$ on $B O(k)$ and isomorphisms $\iota_{k}^{*} \xi_{k+1} \cong \xi_{k} \oplus B O(k) \times \mathbb{R}$ for all $k \in \mathbb{N}$.

We consider a map of spaces $B \rightarrow B \operatorname{Spin}^{c}$ and the corresponding Thom spectrum $M B$. Cycles for elements of the $B$-bordism group $\pi_{n}(M B)$ of $X$ are pairs $(M, f)$ consisting of a closed $n$-dimensional Riemannian manifold $M$ and a stable normal $B$-structure on the map $f: M \rightarrow B$. The additional data of a stable normal $B$ structure is not written explicitly and fixes the relation between the map $f$ and the tangent bundle of $M$. In the following we explain the details. Since $M$ is compact there exists a factorisation

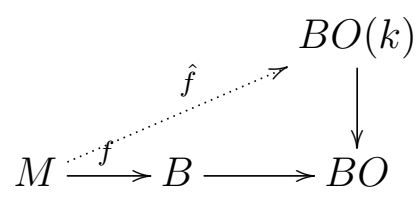

up to homotopy for a suitable integer $k$. We require that $\hat{f}^{*} \xi_{k}$ is a complement of the tangent bundle of $M$, i.e. that there exists an isomorphism

$$
T M \oplus \hat{f}^{*} \xi_{k} \cong M \times \mathbb{R}^{n+k}
$$

Definition 3.1 A normal B-structure on $f$ consists of the choice of $\hat{f}$ and the isomorphism (15).

There is an obvious notion of a stabilisation of a normal $B$-structure which allows to increase $k$. A stable normal $B$-structure is an equivalence class of normal $B$-structures under the relation generated by stabilisation.

The bordism group $\pi_{n}(M B)$ is the set of equivalence classes of cycles, where the equivalence relation is given by bordism, and the group structure is induced by the disjoint sum. A zero bordism of $(M, f)$ is given by a pair $(W, F)$ of similar data, where $W$ is a compact $n+1$-dimensional Riemannian manifold with boundary $\partial W \cong M$ and product structure and $F: W \rightarrow B$ carries a stable normal $B$-structure which extends the one on $f$. In detail this means the following. First of all the map $\hat{F}$ extends $\hat{f}$. The stable normal $B$-structure on $W$ is represented by an isomorphism

$$
T W \oplus \hat{F}^{*} \xi_{k} \cong W \times \mathbb{R}^{n+1+k} .
$$

The outgoing normal field of $T W_{\mid \partial W}$ provides an orthogonal decomposition

$$
T W_{\mid M} \cong T M \oplus(M \times \mathbb{R}) .
$$


It is here where we use the additional datum of the Riemannian metrics in order to rigidify the choice of the unit normal vector field. We require that the isomorphism

$$
T M \oplus(M \times \mathbb{R}) \oplus \hat{f}^{*} \xi_{k} \stackrel{\sqrt{17}}{=} T W_{\mid M} \oplus \hat{f}^{*} \xi_{k} \stackrel{\sqrt[16]{ } \stackrel{1}{I}^{M}}{\cong} M \times \mathbb{R}^{n+1+k}
$$

represents the stable normal $B$-structure on $f$.

\subsection{Normal and tangential $S p i n^{c}$-structures}

Because of the factorisation $B \rightarrow B$ Spin $^{c} \rightarrow B O$ a normal $B$-structure induces a normal $S_{p i n}{ }^{c}$-structure. As we will do geometry on the tangent bundle we must transfer normal $S_{p i n}{ }^{c}$-structures to tangential Spin ${ }^{c}$-structures. The homotopy theoretic picture of this transition is explained in [21, Sec. 8] in the example of String-structures. In the following we describe its geometric counterpart.

Let $V \rightarrow M$ be an $m$-dimensional real vector bundle. Then a $\operatorname{Spin}^{c}$-structure on $V$ is pair $(P, \kappa)$, where $P \rightarrow M$ is a $\operatorname{Spin}^{c}(m)$-principal bundle and $\kappa$ is an isomorphism of real vector bundles

$$
\kappa: P \times_{\operatorname{Spin}^{c}(n)} \mathbb{R}^{m} \cong V .
$$

With this definition a $\operatorname{Spin}^{c}$-structure induces an euclidean metric and an orientation on $V$ so that the oriented orthonormal frame bundle is $S O(V):=P \times_{\operatorname{Spin}^{c}(m)} S O(m)$.

The collection of all $S p i n^{c}$-structures on the vector bundle $V$ naturally forms a groupoid $\operatorname{Spin}^{c}(V)$. The objects of the groupoid $\operatorname{Spin}^{c}(V)$ are the $\operatorname{Spin}^{c}$-structures $(P, \kappa)$, and the morphisms $(P, \kappa) \rightarrow\left(P^{\prime}, \kappa^{\prime}\right)$ are isomorphisms of $\operatorname{Spin}^{c}(m)$-principal bundles $P \rightarrow P^{\prime}$ which are compatible with the isomorphisms $\kappa$ and $\kappa^{\prime}$. This in particular implies that automorphisms of $(P, \kappa)$ are given by the central action of $C^{\infty}(M, U(1))$ on $P$.

If we associate to any open subset $A \subseteq M$ the groupoid $\operatorname{Spin}^{c}\left(V_{\mid A}\right)$, then we obtain a sheaf of groupoids $\operatorname{Spin}^{c}(V)$ which actually is an $U(1)$-banded gerbe. We refer to [18, [46], or [33] for an introduction to gerbes. Isomorphism classes of $U(1)$-banded gerbes $\mathcal{G}$ are classified by their Dixmier-Douady classes $\operatorname{DD}(\mathcal{G}) \in H^{3}(M ; \mathbb{Z})$. In particular, the DixmierDouday class of the $\operatorname{Spin}^{c}$-gerbe $\operatorname{Spin}^{c}(V)$ is the class

$$
\mathrm{DD}\left(\underline{\left.\operatorname{Spin}^{c}(V)\right)}\right)=W_{3}(V)=\beta\left(w_{2}(V)\right) \in H^{3}(M ; \mathbb{Z}),
$$

where $w_{2}(V) \in H^{2}(M ; \mathbb{Z} / 2 \mathbb{Z})$ is the second Stiefel-Whitney class and $\beta: H^{2}(M ; \mathbb{Z} / 2 \mathbb{Z}) \rightarrow$ $H^{3}(M ; \mathbb{Z})$ is the Bockstein operator [43, Thm. D2]. The groupoid $\operatorname{Spin}^{c}(V)$ is non-empty if and only if $W_{3}(V)=0$, i.e. the class $W_{3}(V)$ is the obstruction against the existence of a $\operatorname{Spin}^{c}$-structure on $V$. In the following we will simplify the notation and write $P$ for the $\operatorname{Spin}^{c}$-structure $(P, \kappa)$.

Let $\mathcal{B} U(1)(M)$ denote the Picard groupoid (see [25]) of $U(1)$-principal bundles on $M$. Given an $U(1)$-principal bundle $E \in \mathcal{B} U(1)(M)$ and a $\operatorname{Spin}^{c}$-structure $P \in \operatorname{Spin}^{c}(V)$, we can define a new $\operatorname{Spin}^{c}$-structure $E \otimes P \in \operatorname{Spin}^{c}(V)$. A formula for this tensor product is given by (20) specialised to the case $n=0$, see below. This construction defines bifunctor

$$
\mathcal{B} U(1)(M) \times \operatorname{Spin}^{c}(V) \rightarrow \operatorname{Spin}^{c}(V) .
$$


If $\operatorname{Spin}^{c}(V)$ is not empty, then the set of isomorphism classes of $\operatorname{Spin}^{c}$-structures on $V$ is a torsor over the group of isomorphism classes in $\mathcal{B U}(1)(M)$. Since the latter is canonically isomorphic to $H^{2}(M ; \mathbb{Z})$ we get a simply transitive action of $H^{2}(M ; \mathbb{Z})$ on $\operatorname{Spin}^{c}(V) /$ iso. Furthermore, we get natural isomorphisms

$$
C^{\infty}(M, U(1)) \cong \operatorname{Aut}_{\mathcal{B} U(1)(M)}(E) \cong \operatorname{Aut}_{\operatorname{Spin}^{c}(V)}(E \otimes P) \cong \operatorname{Aut}_{\operatorname{Spin}^{c}(V)}(P) .
$$

The sum of two vector bundles with $\operatorname{Spin}^{c}$-structures has a naturally induced Spin ${ }^{c}$ structure. This is formalised as the natural bifunctor

$$
\operatorname{Spin}^{c}(V) \times \operatorname{Spin}^{c}(U) \rightarrow \operatorname{Spin}^{c}(V \oplus U)
$$

On the level of objects this bifunctor is given by

$$
(P, Q) \mapsto P \otimes Q
$$

where the $\operatorname{Spin}^{c}(n+m)$-principal bundle

$$
P \otimes Q:=\left(P \times_{M} Q\right) \times_{\left(\operatorname{Spin}^{c}(n) \times \operatorname{Spin}^{c}(m)\right)} \operatorname{Spin}^{c}(n+m)
$$

is obtained from the $\operatorname{Spin}^{c}(n) \times \operatorname{Spin}^{c}(m)$-principal bundle $P \times_{M} Q$ by extension of structure groups along the upper horizontal map in the diagram

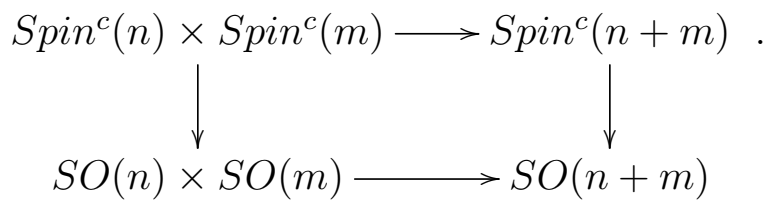

Here $n=\operatorname{dim}(V)$ and $m=\operatorname{dim}(U)$, and the compatibility with the lower part of this diagram is used to define the structure map $\kappa_{P \otimes Q}$ from $\kappa_{P}$ and $\kappa_{Q}$. The bifunctor comes equipped with natural associativity constraints. We omit the details of the latter two aspects.

We set $\operatorname{Spin}^{c}(0):=U(1)$ and let $0_{M}$ denote the zero dimensional vector bundle on $M$. Then we get an identification of $\operatorname{Spin}^{c}\left(0_{M}\right) \cong \mathcal{B U}(1)(M)$, and for $n=0$ the bifunctor (19) specialises to (18). As a consequence of associativity the bifunctor (19) is compatible with the action 18$)$ of $\mathcal{B} U(1)(M)$ in the sense that for $E \in \mathcal{B} U(1)(M)$ have natural isomorphisms

$$
(E \otimes P) \otimes Q \cong E \otimes(P \otimes Q) \cong P \otimes(E \otimes Q) .
$$

The trivialised vector bundle $M \times \mathbb{R}^{n}$ has a preferred trivial $\operatorname{Spin}^{c}$-structure $Q(n):=$ $M \times \operatorname{Spin}^{c}(n)$. We can use this to produce a canonical equivalence of groupoids

$$
\operatorname{Spin}^{c}(V) \cong \operatorname{Spin}^{c}\left(V \oplus\left(M \times \mathbb{R}^{n}\right)\right), \quad P \mapsto P \otimes Q(n) .
$$

On the level of $\operatorname{Spin}^{c}$-structures we speak of stabilisations. 
Let us now consider a pair $(M, f)$ of a compact $n$-dimensional Riemannian manifold and a map $f: M \rightarrow B$ which admits a refinement to a stable normal $B$-structure. Then we can assume that $f$ has a factorisation up to homotopy over $B \operatorname{Spin}^{c}(k)$ as in the diagram

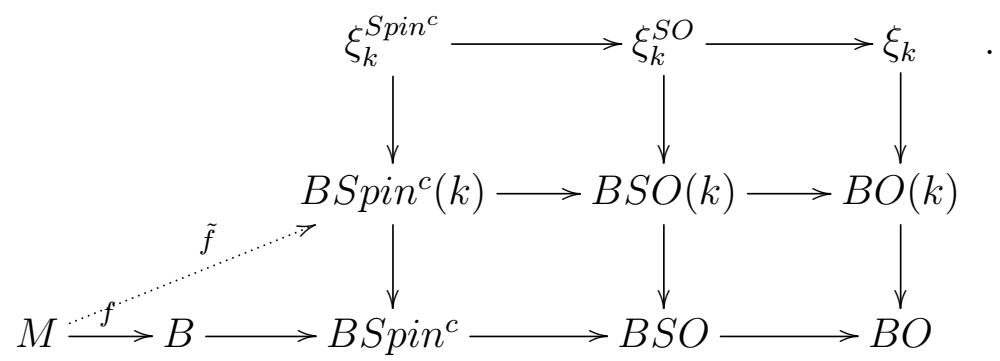

The map $\tilde{f}$ classifies a $\operatorname{Spin}^{c}(k)$-principal bundle $\tilde{f}^{*} Q_{k} \rightarrow M$, where $Q_{k} \rightarrow B \operatorname{Spin}^{c}(k)$ denotes the universal $\operatorname{Spin}^{c}(k)$-bundle. Note that we have $\tilde{f}^{*} Q_{k} \in \operatorname{Spin}^{c}\left(\tilde{f}^{*} \xi_{k}^{\operatorname{Spin}^{c}}\right)$.

We let $\hat{f}: M \rightarrow B O(k)$ be induced by $\tilde{f}$ so that $\hat{f}^{*} \xi_{k} \cong \tilde{f}^{*} \xi_{k}^{S p i n}{ }^{c}$. With these identifications the trivialisation 15 induces a bifunctor 19

$$
\operatorname{Spin}^{c}(T M) \times \operatorname{Spin}^{c}\left(\tilde{f}^{*} \xi_{k}^{\operatorname{Spin}^{c}}\right) \rightarrow \operatorname{Spin}^{c}\left(M \times \mathbb{R}^{n+k}\right) .
$$

Since $\mathcal{B U}(1)(M)$ acts simply transitively on isomorphisms classes we conclude using (21) that there is a unique isomorphism class of $\operatorname{Spin}^{c}$-structures $P \in \operatorname{Spin}^{c}(T M)$ such that

$$
P \otimes \tilde{f}^{*} Q_{k} \cong Q(n+k) \text {. }
$$

One can further check that this isomorphism class only depends on the normal $B$-structure represented by $f$ and not on its representative. This is the tangential $\operatorname{Spin}^{c}$-structure determined by the normal Spin $^{c}$-structure.

For constructions which involve glueing or in the notion of a $\operatorname{Spin}^{c}$-map we need a rigidified notion of a tangential $\operatorname{Spin}^{c}$-structure.

Definition 3.2 Assume that we have fixed a normal B-structure in terms of the factorisation $\tilde{f}$ and the isomorphism (15). Then we define a tangential Spin ${ }^{c}$-structure as a pair of a Spin ${ }^{c}$-structure $P \in \operatorname{Spin}^{c}(T M)$ together with a choice of an isomorphism in (23).

There are many tangential Spin ${ }^{c}$-structures associated to a normal Spin ${ }^{c}$-structure, but the main point is that two of them are isomorphic by a canonical isomorphism.

Let $h: M \rightarrow W$ be a smooth map and assume that we are given oriented euclidean vector bundles $V_{M} \rightarrow M$ and $V_{W} \rightarrow W$ together with an isomorphism

$$
V_{M} \oplus\left(M \times \mathbb{R}^{k}\right) \cong h^{*} V_{W} \oplus\left(M \times \mathbb{R}^{l}\right) .
$$

Assume further that we are given $\operatorname{Spin}^{c}$-structures $P_{M} \in \operatorname{Spin}^{c}\left(V_{M}\right)$ and $P_{W} \in \operatorname{Spin}^{c}\left(V_{W}\right)$.

Definition 3.3 A a refinement of $h$ to a Spinc-map is a choice of an isomorphism

$$
h^{*} P_{W} \otimes Q(l) \cong P_{M} \otimes Q(k)
$$

in $\operatorname{Spin}^{c}\left(V_{M} \oplus\left(M \times \mathbb{R}^{k}\right)\right)$ (this uses (24)). The equivalence class of a Spinc-map under stabilisation will be called a stable Spin ${ }^{c}$-map. 
Being a $\operatorname{Spin}^{c}$-map is an additional datum, not just a property of the map. Observe that we can compose stable $\operatorname{Spin}^{c}$-maps in a natural way.

We now assume that $(W, F)$ is a zero bordism of $(M, f)$. We choose a representative of the normal $B$-structure on $W$ involving the factorisation $\tilde{F}: W \rightarrow \operatorname{BSpin}^{c}(k)$. On $M$ we take the induced factorisation $\tilde{f}:=\tilde{F}_{\mid M}$. Then we have a natural decomposition of oriented euclidean vector bundles

$$
T W_{\mid M} \cong T M \oplus(M \times \mathbb{R}),
$$

where we trivialise the normal bundle by the outgoing unit normal vector field. Assume now that we have chosen tangential $S_{p i n}{ }^{c}$-structures $P(T M)$ and $P(T W)$ on $M$ and $W$, respectively (see Definition 3.2). In this situation we get a natural refinement of the inclusion $M \rightarrow W$ to a $\operatorname{Spin}^{c}$-map. This refinement is distinguished by the condition that the following diagram in $\operatorname{Spin}^{c}\left(M \times \mathbb{R}^{n+1+k}\right)$

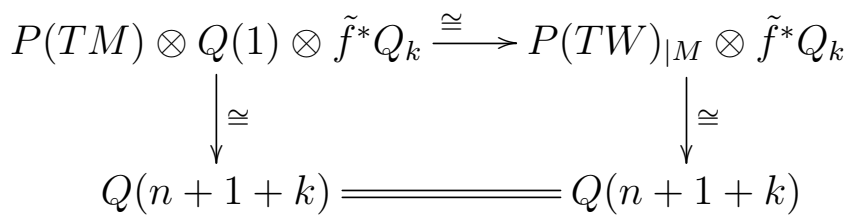

commutes. Here the upper corners are interpreted in $\operatorname{Spin}^{c}\left(M \times \mathbb{R}^{n+1+k}\right)$ using the normal $B$-structures on $M$ or $W$, respectively. The vertical morphisms are given by the tangential Spin ${ }^{c}$-structures. Finally, the upper horizontal isomorphism uses (26) and fixes the refinement of the inclusion $M \rightarrow W$ to a $\operatorname{Spin}^{c}$-map.

\subsection{The definition of $\eta^{a n}$}

We consider a cycle $(M, f)$ for a class $x=[M, f] \in \pi_{n}(M B)$ and assume in addition that $x$ is torsion. Then there exists a non-zero integer $l \in \mathbb{N}$ such that $l x=0$. We can thus find a zero bordism $(W, F)$ of the disjoint union of $l$ copies of $(M, f)$ which we denote by $l(M, f)$.

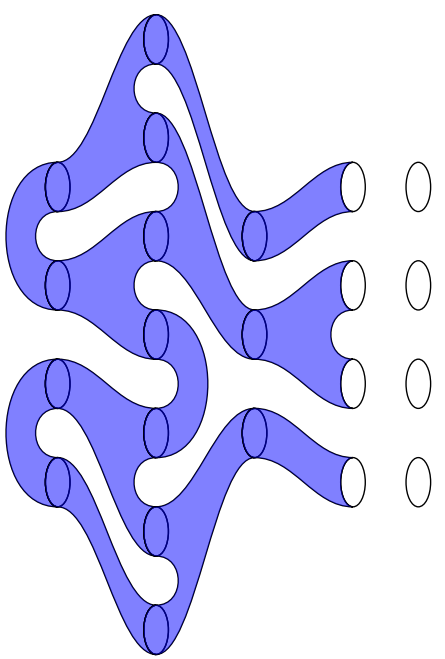


A picture of $M$ and the zero bordism $W$ of $4 M$

We will define $\eta^{a n}(x) \in Q_{n}(B)$ in terms of a collection of indices of associated $\mathbb{Z} / l \mathbb{Z}$ index problems [30]. In order to formulate these index problems and to express the indices in terms of geometric and spectral invariants we must choose appropriate geometric structures.

We choose a tangential $\operatorname{Spin}^{c}$-structure $(P, \kappa) \in \operatorname{Spin}^{c}(T M)$ related to the stable normal $\operatorname{Spin}^{c}$-structure on $f$. A connection $\tilde{\nabla}^{T M}$ on $P$ induces via $\kappa$ a connection on $T M$. We say that $\tilde{\nabla}^{T M}$ is a $S p i n^{c}$-extension of the Levi-Civita connection on $M$ if it induces the Levi-Civita connection $\nabla^{T M, L C}$ on $T M$.

The group $\operatorname{Spin}^{c}(n)$ has a distinguished unitary representation called the spinor representation $\Delta^{n}$. For even $n$ its dimension is $2^{n / 2}$, and it has a decomposition $\Delta^{n} \cong \Delta^{n,+} \oplus \Delta^{n,-}$. It is related with the odd-dimensional case by $\Delta_{\mid \operatorname{Spin}^{c}(n-1)}^{n,+} \cong \Delta^{n-1}$.

The bundle $S(T M):=P \times_{\operatorname{Spin}^{c}(n)} \Delta^{n} \rightarrow M$ is called the Spinor bundle of $M$. If we have chosen a $S p i n^{c}$-extension $\tilde{\nabla}^{T M}$ of the Levi-Civita connection on $M$, then the spinor bundle carries the structure of a Dirac bundle. We thus obtain the Spin ${ }^{c}$-Dirac operator $\not D_{M}$ which acts on sections of $S(T M)$. Standard references for these constructions are [14, Ch. 3], [43, App. D].

If we are given a class $\phi \in K^{0}(B)$, then we can choose a $\mathbb{Z} / 2 \mathbb{Z}$-graded vector bundle $V \rightarrow$ $M$ whose $K$-theory class satisfies $[V]=f^{*} \phi \in K^{0}(M)$. We choose a hermitean metric $h^{V}$ and a metric connection $\nabla^{V}$ which preserve the grading. The triple $\mathbf{V}:=\left(V, h^{V}, \nabla^{V}\right)$ will then be called a geometric vector bundle. We let $D_{M} \otimes \mathbf{V}$ be the Dirac operator twisted by $\mathbf{V}$. It acts on sections of $S(T M) \otimes V$.

We now assume that $n=\operatorname{dim}(M)$ is odd. The $\eta$-invariant [5] of the twisted Dirac operator

$$
\eta\left(\not D_{M} \otimes \mathbf{V}\right) \in \mathbb{R}
$$

is defined as the value at $s=0$ of the meromorphic continuation of the $\eta$-function function

$$
\eta\left(\not D_{M} \otimes \mathbf{V}\right)(s):=\operatorname{Tr}_{s}\left|\not D_{M} \otimes \mathbf{V}\right|^{-s} \operatorname{sign}\left(\not D_{M} \otimes \mathbf{V}\right)
$$

where $\operatorname{Tr}_{s}$ is the super trace with respect to the grading of $V$. Note that the trace exists if $\operatorname{Re}(s)>n$, and that the meromorphic continuation of the $\eta$-function is regular at $s=0$ by the results of [5]. The $\eta$-invariant depends on the geometry of $M$ and $V$ in a possibly discontinuous way with jumps when eigenvalues of $D_{M} \otimes \mathbf{V}$ cross zero. In order to get a quantity which depends continuously on the geometry one usually considers the reduced $\eta$-invariant for which in the present paper we will use the symbol $\xi$ :

$$
\xi\left(\not D_{M} \otimes \mathbf{V}\right):=\left[\frac{\eta\left(\not_{M} \otimes \mathbf{V}\right)+\operatorname{dim} \operatorname{ker}\left(\not D_{M} \otimes \mathbf{V}\right)}{2}\right] \in \mathbb{R} / \mathbb{Z}
$$

In an appropriate model of $\pi_{n}(M B \mathbb{Q} / \mathbb{Z})$ the zero bordism $(W, F)$ of $l(M, f)$ geometrically represents the lift of $x$ to a class

$$
\hat{x}=[W, F] \in \pi_{n+1}(M B \mathbb{Q} / \mathbb{Z}),
$$


using the notation of the diagram (3). We refer to Lemma 3.7 for more details. We choose a $\operatorname{Spin}^{c}$-connection $\tilde{\nabla}^{T W}$ extending the Levi-Civita connection on $T W$ which extends the connection on the boundary of $W$ induced by $\tilde{\nabla}^{T M}$. The class $F^{*} \phi \in K^{0}(W)$ extends the class $F_{\mid \partial W}^{*} \phi \in K^{0}(\partial W)$ which restricts to $f^{*} \phi \in K^{0}(M)$ on the copies of $M$ in the boundary of $W$. Hence we can assume, after adding some trivial bundles to the even and odd parts of $V$, that the bundle on $\partial W$ induced by $V$ has an extension $U$ to $W$. We choose a hermitean metric $h^{U}$ and a metric connection $\nabla^{U}$ on $U$ which extend the already given data on the boundary. In this way we get a geometric bundle $\mathbf{U}:=\left(U, h^{U}, \nabla^{U}\right)$.

We can now form the Atiyah-Patodi-Singer boundary value problem for $\not_{W} \otimes \mathbf{U}$. The analytic details of that boundary value problem are not important for our present purpose so that we refer to [5] for a precise description. We only have to know that it produces a Fredholm operator $\left(\not D_{W} \otimes \mathbf{U}\right)_{A P S}$ which has a well-defined index

$$
\operatorname{index}\left(\left(D_{W} \otimes \mathbf{U}\right)_{A P S}\right) \in \mathbb{Z},
$$

and that the following index formula proved in [5] holds true:

$\operatorname{index}\left(\left(\not D_{W} \otimes \mathbf{U}\right)_{A P S}\right)=\int_{W} p_{n+1}\left(\mathbf{T d}\left(\tilde{\nabla}^{T W}\right) \wedge \mathbf{c h}\left(\nabla^{U}\right)\right)-l \frac{\eta\left(\not D_{M} \otimes \mathbf{V}\right)+\operatorname{dim} \operatorname{ker}\left(\not D_{M} \otimes \mathbf{V}\right)}{2}$

In this formula the closed form $\mathbf{T} \mathbf{d}\left(\tilde{\nabla}^{T W}\right) \in \Omega^{0}(W)\left[b, b^{-1}\right]$ is the Chern-Weyl representative determined by the universal class $\mathbf{T d} \in H P \mathbb{Q}^{0}\left(B \operatorname{Spin}^{c}\right)$ and the connection $\tilde{\nabla}^{T M}$. Similarly, the form $\operatorname{ch}\left(\nabla^{U}\right) \in \Omega^{0}(W)\left[b, b^{-1}\right]$ is the Chern-Weyl representative determined by the class $\mathbf{c h} \in H P \mathbb{Q}^{0}(B U)$ and the connection $\nabla^{U}$. Note that we use powers of $b$ in order to shift the higher form-degree components to total degree zero.

We consider the element

$$
e:=\left[\frac{\operatorname{index}\left(D_{W} \otimes \mathbf{U}\right)_{A P S}}{l}\right] \in \mathbb{Q} / \mathbb{Z}
$$

Equivalently, by the index theorem 28 and 27 we can write

$$
e=\left[\frac{1}{l} \int_{W} p_{n+1}\left(\mathbf{T d}\left(\tilde{\nabla}^{T W}\right) \wedge \mathbf{c h}\left(\nabla^{U}\right)\right)\right]-\xi\left(\not D_{M} \otimes \mathbf{V}\right)
$$

if we interpret this equality in $\mathbb{R} / \mathbb{Z}$. The quantity $e$ can be interpreted as a $\mathbb{Z} / l \mathbb{Z}$-index in the sense of [30]. In the following proposition we state how the number $e$ depends on the data.

Proposition 3.4 1. The value of e does not depend on the choices of the geometric structures on $M$ and $W$.

2. The value of e only depends on the $K$-theory class $\phi$. This dependence is additive and determines an element $\tilde{e} \in \operatorname{Hom}^{\text {cont }}\left(K^{0}(B), \mathbb{Q} / \mathbb{Z}\right)$.

3. The class $[\tilde{e}] \in Q_{n}(M B)$ of this homomorphism (using the presentation (12)) does not depend on $l$ or the choice of the zero bordism of $(W, F)$. 
4. The element $[\tilde{e}] \in Q_{n}(M B)$ described in 3. only depends on the bordism class $x$. This dependence is additive so that we obtain a well-defined homomorphism

$$
\eta^{a n}: \pi_{n}(M B)_{t o r s} \rightarrow Q_{n}(M B) .
$$

Proof. On the one hand, we have $e \in \frac{1}{l} \mathbb{Z} / \mathbb{Z} \subseteq \mathbb{R} / \mathbb{Z}$. On the other hand, we know that the right-hand side of $(30)$ depends continuously on the geometric data. This shows that $e$ does not depend on the geometric structures at all since two choices of geometric structures can be connected by a family. This proves 1 .

The element $e$ depends additively on the bundle $U$ and therefore only depends on the class $[U] \in K^{0}(M)$. Its construction therefore induces a homomorphism $\tilde{e} \in \operatorname{Hom}\left(K^{0}(B), \mathbb{Q} / \mathbb{Z}\right)$. Since it factors over the restriction along the map $f: M \rightarrow B$ and $M$ is compact this homomorphism is continuous. We use the identification

$$
\mathbb{Q} / \mathbb{Z} \cong \pi_{0}(K \mathbb{Q} / \mathbb{Z}) \stackrel{b^{-\frac{n+1}{2}}}{\longrightarrow} \pi_{n+1}(K \mathbb{Q} / \mathbb{Z})
$$

in order to interpret $\tilde{e}$ as an element of $\operatorname{Hom}^{\text {cont }}\left(K^{0}(B), \pi_{n+1}(K \mathbb{Q} / \mathbb{Z})\right)$. This shows 2 .

Assume that we have a second zero bordism $\left(W^{\prime}, F^{\prime}\right)$ of $l^{\prime}(M, f)$ yielding a homomorphism $\tilde{e}^{\prime} \in \operatorname{Hom}^{\text {cont }}\left(K^{0}(B), \mathbb{Q} / \mathbb{Z}\right)$. Then by glueing along boundary components we can form the closed Riemannian $n+1$-dimensional $B$-manifold $\tilde{W}:=l^{\prime} W \cup_{l l^{\prime} M} l W^{\prime}$ which comes with a map $\tilde{F}: \tilde{W} \rightarrow B$. The latter has a natural refinement to a stable normal $B$-structure which restricts to the given stable normal $B$-structures on $W$ and $W^{\prime}$.
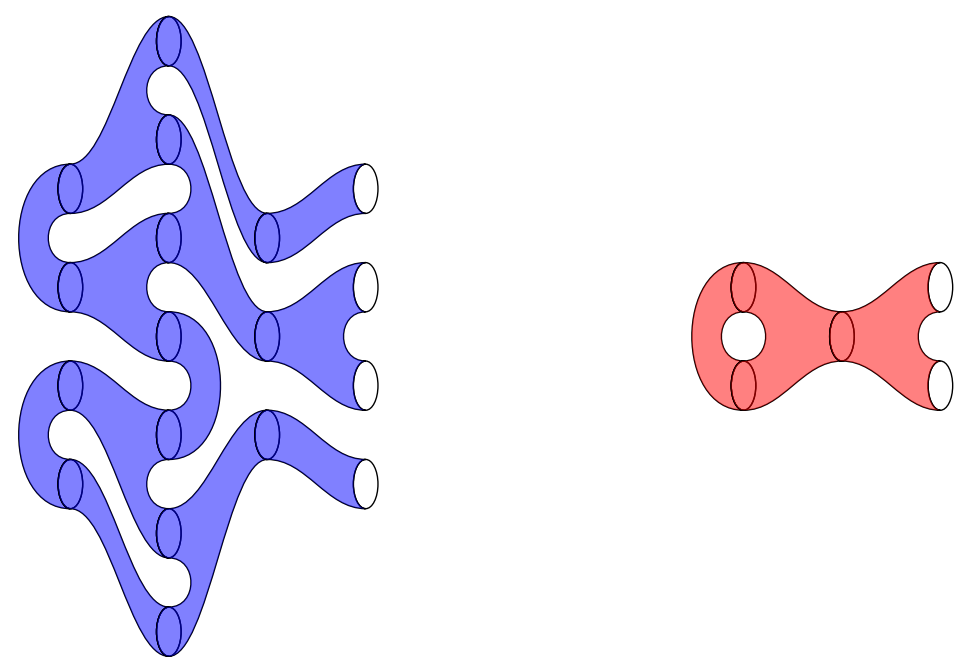


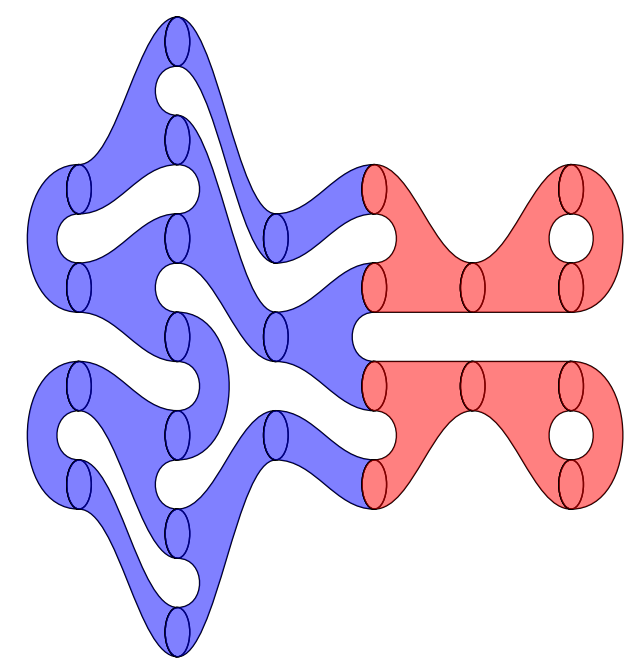

Pictures of $W, W^{\prime}$, and $\frac{1}{2} \tilde{W}$ with $l=4$ and $l^{\prime}=2$

Note that the tangential $\operatorname{Spin}^{c}$-structures $(P, \kappa)$ and $\left(P^{\prime}, \kappa^{\prime}\right)$ come with isomorphisms of the type (23). Compatibility with these fixes the morphism which we have to use order to glue $P$ with $P^{\prime}$. In this way we get a tangential $\operatorname{Spin}^{c}$-structure on $\tilde{W}$. The triple $(\tilde{W}, \tilde{F})$ is thus cycle for a class

$$
y:=[\tilde{W}, \tilde{F}] \in \pi_{n+1}(M B) .
$$

Then for $\phi \in K^{0}(B)$ we get from the right-hand side of 30 that

$$
\tilde{e}(\phi)-\tilde{e}^{\prime}(\phi)=\left[\frac{1}{l l^{\prime}}\left\langle\mathbf{T d}(T \tilde{W}) \cup \tilde{F}^{*} \mathbf{c h}(\phi),[\tilde{W}]\right\rangle\right] .
$$

Since $\tilde{F}^{*} \mathbf{T} \mathbf{d}^{-1}=\mathbf{T} \mathbf{d}(T \tilde{W})$ this is exactly the formula 13$)$ for the evaluation of $\epsilon\left(\frac{1}{l l^{\prime}} y\right) \in$ $\pi_{n+1}(K \wedge M B \mathbb{Q})$ against $\operatorname{Thom}^{K}(\phi) \in K^{0}(M B)$. Therefore the class $[\tilde{e}] \in Q_{n}(M X)$ is independent of the choice of $l$ and the zero bordism $(W, F)$. This finishes the verification of 3 .

We observe that the map which associates to $(M, f)$ the class $[\tilde{e}] \in Q_{n}(M B)$ is additive under disjoint unions. Moreover, if $(M, f)$ itself is zero bordant, i.e. we can find $(W, F)$ as above with $l=1$, then $[\tilde{e}]=0$. It follows that the construction above uniquely descends to a homomorphism

$$
\eta^{a n}: \pi_{n}(M B)_{\text {tors }} \rightarrow Q_{n}(M B)
$$

Let us collect the essentials of this construction in the following definition.

Definition 3.5 We define $\eta^{a n}:=0$ for even $n$. For odd $n$ we define the homomorphism

$$
\eta^{a n}: \pi_{n}(M B)_{\text {tors }} \rightarrow Q_{n}(M B)
$$


by the following prescription: If $x \in \pi_{n}(M B)_{\text {tors }}$ is represented by $(M, f)$, then we choose a zero bordism $(W, F)$ of $l(M, f)$ for a suitable non-zero $l \in \mathbb{N}$. If $\phi \in K^{0}(B)$, then we choose a geometric bundle $\mathbf{U} \rightarrow W$ whose underlying $K$-theory class is $F^{*} \phi \in K^{0}(W)$ and whose restrictions to the l copies of $M$ in the boundary are pairwise isomorphic.

We further choose a Spin ${ }^{c}$-geometry for $W$ such that the restrictions to the $l$ copies of $M$ in the boundary of $W$ are again pairwise isomorphic. Then $\eta^{a n}(x) \in Q_{n}(M B)$ is represented by the homomorphism

$$
K^{0}(B) \ni \phi \mapsto\left[\frac{1}{l} \operatorname{index}\left(\left(D_{W} \otimes \mathbf{U}\right)_{A P S}\right)\right] \in \mathbb{Q} / \mathbb{Z} \cong \pi_{n+1}(K \mathbb{Q} / \mathbb{Z})
$$

\subsection{The secondary index theorem}

In the Definitions 2.3 and 3.5 we have described homomorphisms

$$
\eta^{\text {top }}: \pi_{n}(M B)_{\text {tors }} \rightarrow Q_{n}(M B), \quad \eta^{a n}: \pi_{n}(M B)_{\text {tors }} \rightarrow Q_{n}(M B) .
$$

Both constructions follow a common idea. Given a torsion element $x \in \pi_{n}(M B)_{\text {tors }}$ in a first step a lift $\hat{x} \in \pi_{n}(M B \mathbb{Q} / \mathbb{Z})$, respectively a geometric representative of such a lift, is chosen. The homotopy theoretic invariant $\eta^{\text {top }}(x)$ is represented by the homomorphism $K^{0}(M B) \rightarrow \mathbb{Q} / \mathbb{Z}$ induced by this lift via the homotopy theoretic pairing between $K$-homology and cohomology. The analytic variant $\eta^{a n}(x)$ is represented by a homomorphisms, which this time is obtained from a suitable family of Atiyah-Patodi-Singer index problems on the geometric representative of the lift $\hat{x}$. Because of these coincidences it is very natural to expect that the following theorem holds true.

\section{Theorem 3.6}

$$
\eta^{a n}=\eta^{t o p} .
$$

Proof. An obvious option is to apply the $\mathbb{Z} / l \mathbb{Z}$-index theorem [30] directly to $\eta^{a n}$ in order to express it in homotopy theoretic terms. In this paper we decided to go a different path. It is interesting since it explains in greater detail in which sense the homotopy theoretic construction of $\eta^{t o p}$ and the geometric or analytic constructions involved in $\eta^{a n}$ correspond to each other. Our bride between analysis and topology will be the identification of homotopy theoretic $K$-homology with the analytic picture [13] and the ordinary AtiyahSinger index theorem for elliptic operators [9], respectively its local form described in [14, Ch. IV].

Some ideas of our proof of Theorem 3.6, in particular the usage of Moore spaces, are taken from [30] and the proof of the $\mathbb{R} / \mathbb{Z}$-index theorem [7, Thm 5.3].

We start with a description of Moore spaces for cyclic groups $\mathbb{Z} / l \mathbb{Z}, l \in \mathbb{Z}$. Let $S^{1} \rightarrow S^{1}$ be the $l$-fold covering of the pointed circle. Its mapping cylinder $Z_{l}$ and mapping cone $C_{l}$ fit into the cofibre sequence of pointed spaces

$$
S^{1} \rightarrow Z_{l} \rightarrow C_{l} \stackrel{\partial}{\rightarrow} \Sigma S^{1} \rightarrow \ldots .
$$


Note that the shifted suspension spectrum $\Sigma^{\infty-1} C_{l}$ is then a model for the Moore spectrum $\mathrm{MZ} / l \mathbb{Z}$ discussed in 2.2 . Further note that the inclusion of the cylinder basis $S^{1} \rightarrow Z_{l}$ is a homotopy equivalence. Hence we have equivalences $\Sigma^{\infty-1} Z_{l} \cong \Sigma^{\infty-1} S^{1} \cong \mathrm{MZ}$. Applying the functor $\Sigma^{\infty-1}$ to the sequence (33) and using these identifications we get the fibre sequence

$$
\mathrm{M} \mathbb{Z} \stackrel{l}{\rightarrow} \mathrm{M} \mathbb{Z} \rightarrow \mathrm{M} \mathbb{Z} / l \mathbb{Z} \stackrel{\partial}{\rightarrow} \Sigma \mathrm{M} \mathbb{Z}
$$

of Moore spectra. We use the Moore spectra $M \mathbb{Z} / l \mathbb{Z}$ and the sequence (34) as approximations for $\mathbb{M Q} / \mathbb{Z}$ by spectra with finite skeleta and $(2)$ in the sense that

$$
\mathrm{MQ} / \mathbb{Z} \cong \operatorname{hocolim}_{l} \mathrm{MZ} / l \mathbb{Z} \text {. }
$$

The connecting maps are fixed by their compatibility with the inclusions

$$
\mathbb{Z} / l \mathbb{Z} \rightarrow \mathbb{Q} / \mathbb{Z}, \quad[n] \mapsto\left[\frac{n}{l}\right] .
$$

Smashing the sequence (34) with $M B$ and taking homotopy groups we get a long exact sequence of abelian groups

$$
\cdots \rightarrow \pi_{n+1}(M B \mathbb{Z} / l \mathbb{Z}) \stackrel{\partial}{\rightarrow} \pi_{n}(M B) \stackrel{l}{\rightarrow} \pi_{n}(M B) \rightarrow \pi_{n}(M B \mathbb{Z} / l \mathbb{Z}) \rightarrow \ldots .
$$

The spectrum $M B \wedge X$ is related with Thom spectra by a fibre sequence

$$
M B \rightarrow M(B \times X) \stackrel{\iota}{\rightarrow} M B \wedge X \rightarrow \Sigma M B,
$$

where we use the structure map $B \times X \stackrel{\mathrm{pr}}{\rightarrow} B \rightarrow B G L(\mathbb{R})$ in order to define the Thom spectrum $M(B \times X)$ and the map $M B \rightarrow M(B \times X)$ is induced by the base point of $X$. We further have an equivalence of spectra

$$
\Sigma M B \mathbb{Z} / l \mathbb{Z} \cong M B \wedge C_{l} .
$$

We use the notation $(F, G)$ in order to write maps from $M$ to $B \times C_{l}$. In the following we construct a cycle $(\tilde{W},(\tilde{F}, \tilde{G}))$ for a class in $\pi_{n+2}\left(M\left(B \times C_{l}\right)\right)$ such that

$$
\iota[\tilde{W},(\tilde{F}, \tilde{G})]=\hat{x} \in \pi_{n+1}(M B \mathbb{Z} / l \mathbb{Z})
$$

under the identification (37), where $\tilde{F}: \tilde{W} \rightarrow B$ is the underlying map of a $B$-structure and $\tilde{G}: \tilde{W} \rightarrow C_{l}$. We will obtain $(\tilde{W},(\tilde{F}, \tilde{G}))$ by closing up the boundary of the triple $(W,(F, G))$ found in 3.4 . 


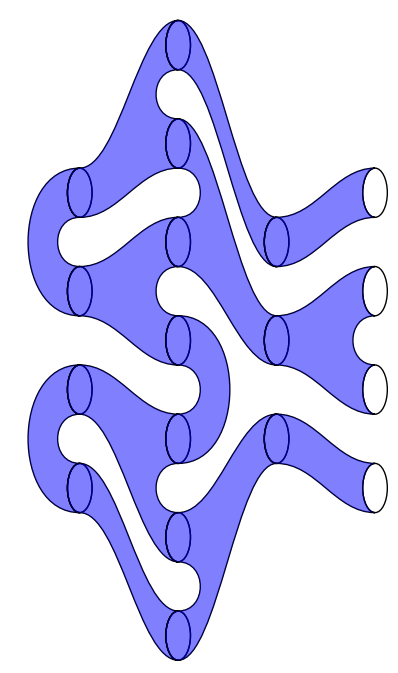

A picture of $S^{1} \times W$

The details are as follows. We consider a two-sphere $S_{l}^{2}$ with $l$ holes.

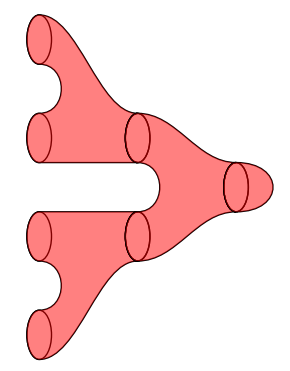

A picture of $S_{4}^{2} \times M$

More precisely we let $S_{l}^{2} \subset S^{2}$ be the compact submanifold with boundary $\partial S_{l}^{2} \cong \bigsqcup_{i=1}^{l} S^{1}$ obtained by deleting the interiors of $l$ disjoint discs from $S^{2}$. We equip $S_{l}^{2}$ with a Riemannian metric with product structure such that all boundary components are isometric to the standard $S^{1}$. The identification of the boundary with the $l$ copies of $S^{1}$ is fixed such that it preserves the natural orientations. We now have an identification

$$
\partial\left(S^{1} \times W\right) \cong l\left(S^{1} \times M\right) \cong \partial\left(S_{l}^{2} \times M\right) .
$$

We let

$$
\tilde{W}:=\left(S^{1} \times W\right) \cup_{l\left(S^{1} \times M\right)}\left(S_{l}^{2} \times M\right)
$$

be the manifold obtained by glueing along the boundary. 


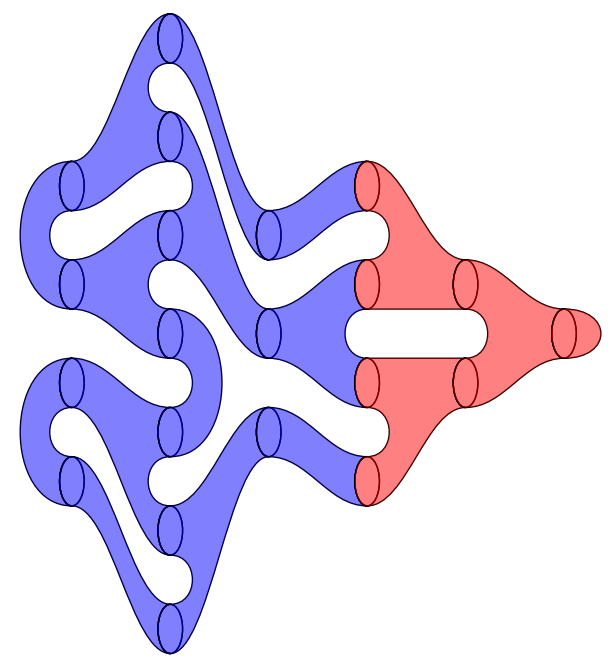

A picture of $\tilde{W}$

We define $\tilde{F}: \tilde{W} \rightarrow B$ such that it restricts to

$$
S^{1} \times W \stackrel{\mathrm{pr}_{W}}{\rightarrow} W \stackrel{F}{\rightarrow} B, \quad S_{l}^{2} \times M \stackrel{\mathrm{pr}_{M}}{\rightarrow} M \stackrel{f}{\rightarrow} B .
$$

We must refine the map $\tilde{F}$ to a normal $B$-structure. We start with the usual normal framing

$$
T S^{2} \oplus S^{2} \times \mathbb{R} \cong S^{2} \times \mathbb{R}^{3}
$$

of $S^{2}$. If we take $k=1$ and let $f$ and $\hat{f}$ in 15 be the constant maps, then we can interpret this isomorphism as a normal $B$-structure, too. By restriction we obtain a normal $B$-structure on $S_{l}^{2}$. Furthermore, the construction explained at the end of 3.2 provides normal $B$-structures

$$
T S^{1} \oplus S^{1} \times \mathbb{R}^{2} \cong S^{1} \times \mathbb{R}^{3}
$$

on the $l$ copies of $S^{1}$ in the boundary of $S_{l}^{2}$ which are isomorphic to each other. We are given a normal $B$-structure

$$
T W \oplus \hat{F}^{*} \xi_{k} \cong W \times \mathbb{R}^{n+1+k}
$$

We therefore get an induced normal $B$-structure on the product $S^{1} \times W$ refining $\tilde{F}_{\mid S^{1} \times W}$ : $T\left(S^{1} \times W\right) \oplus \hat{F}^{\prime, *} \xi_{k+2} \cong T S^{1} \oplus T W \oplus S^{1} \times W \times \mathbb{R}^{2} \oplus\left(\hat{F}^{*} \circ \mathrm{pr}_{W}\right)^{*} \xi_{k} \cong S^{1} \times W \times R^{n+k+4}$, where $\hat{F}^{\prime}$ is the two-fold stabilisation of $\hat{F} \circ \mathrm{pr}_{W}$. In a similar manner, using the induced normal $B$ structures on the copies of $M$ in the boundary of $W$ we get a normal $B$-structure

$$
T\left(S_{l}^{2} \times M\right) \oplus \hat{f}^{*} \xi_{k+1} \cong S_{l}^{2} \times M \times \mathbb{R}^{n+k+4}
$$


on the product $S_{l}^{2} \times M$ which refines $\tilde{F}_{\mid S_{l}^{2} \times M}$. These isomorphisms coincide over the locus of glueing $l\left(S^{1} \times M\right)$. Hence we get a refinement of $\tilde{F}$ to a normal $B$-structure.

We now consider the map

$$
S^{1} \times W \stackrel{\mathrm{pr}_{S}}{\rightarrow} S^{1} \stackrel{i}{\rightarrow} C_{l},
$$

where $i: S^{1} \rightarrow C_{l}$ is the identification of $S^{1}$ with the basis of the mapping cone. Note that the map

$$
\sqcup_{j=1}^{l} i: \partial S_{l}^{2} \cong \bigsqcup_{j=1}^{l} S^{1} \rightarrow C_{l}
$$

can be extended to a map

$$
g: S_{l}^{2} \rightarrow C_{l}
$$

We can and will restrict the choice of $g$ such that it is smooth on the preimage of a neighbourhood $U$ of the cone basis $\partial C_{l} \subset C_{l}$, and regular values of $g$ in the interior $U \backslash \partial C_{l}$ have exactly one preimage. The restriction of the map $\sqrt{39}$ to $\partial\left(S^{1} \times W\right) \cong l\left(S^{1} \times M\right)$ thus has an extension across the other part $S_{l}^{2} \times M$ of $\tilde{W}$ given by

$$
S_{l}^{2} \times M \stackrel{\mathrm{pr}^{2}}{\rightarrow} S_{l}^{2} \stackrel{g}{\rightarrow} C_{l}
$$

Altogether we obtain the map $\tilde{G}: \tilde{W} \rightarrow C_{l}$. The cycle $(\tilde{W},(\tilde{F}, \tilde{G}))$ represents a class in in $\pi_{n+2}\left(M\left(B \times C_{l}\right)\right)$ and we consider its image

$$
\hat{x}:=\iota[\tilde{W},(\tilde{F}, \tilde{G})] \in \pi_{n+2}\left(M B \wedge C_{l}\right) \stackrel{\sqrt[377]{=}}{=} \pi_{n+1}(M B \mathbb{Z} / l \mathbb{Z}) .
$$

Let $\partial: \pi_{n+1}(M B \mathbb{Z} / l \mathbb{Z}) \rightarrow \pi_{n}(M B)$ be the boundary as in $(36)$.

Lemma 3.7 We have $\partial \hat{x}=x$

Proof. The boundary operator $\partial$ in the Lemma is induced by the map denoted by the same symbol in 33

$$
\partial: C_{l} \stackrel{p}{\rightarrow} \Sigma S^{1} \cong S^{2}
$$

where $p$ is the projection which contracts the cone basis to a point. Therefore $\partial \hat{x} \in$ $\pi_{n+2}\left(M B \wedge S^{2}\right)$ is represented by $(\tilde{W},(\tilde{F}, p \circ \tilde{G}))$. We must show that it corresponds to $x$ under the suspension isomorphism

$$
\pi_{n}(M B) \cong \pi_{n+2}\left(M B \wedge S^{2}\right)
$$

To this end we invert the suspension isomorphism in the geometric picture. This inverse is of course given by taking the inverse image of a regular point in $S^{2}$ of the corresponding component $p \circ \tilde{G}$ of the structure map. If we take the inverse image of a point in the neighbourhood $U \backslash \partial C_{l}$ mentioned above we exactly recover the representative $(M, f)$ of $x$. 
The construction of $\eta^{\text {top }}$ involves the $K$-homology of a based space $Y$ defined homotopy theoretically as $\pi_{*}(K \wedge Y)$. It is equivalent to the analytic picture introduced in [13]. The analytic $K$-homology is subsumed in the more general bivariant $K K$-theory (see [40] and the text book [15]) which allows to treat $K$-homology and cohomology on equal footing. Of particular importance for our purpose is that the product in $K K$-theory provides a description of the $\cap$-product between $K$-homology and $K$-theory which easily compares with the operation of twisting Dirac operators.

The unit of $K$-theory induces the map

$$
\epsilon: \pi_{n+2}\left(M B \wedge C_{l}\right) \rightarrow \pi_{n+2}\left(K \wedge M B \wedge C_{l}\right)
$$

We use the Thom isomorphism for $M B$ in $K$-homology

$$
\operatorname{Thom}_{K}: \pi_{n+2}\left(K \wedge M B \wedge C_{l}\right) \stackrel{\sim}{\rightarrow} \pi_{n+2}\left(K \wedge B_{+} \wedge C_{l}\right) .
$$

Finally we use $K K$-theory in order represent this $K$-homology of a pointed space analytically. For the moment we assume that $X$ and $B$ are compact. This is no real restriction since we are calculating with a finite number of cycles at a time and their structure maps can only hit compact parts of the spaces $B$ and $X$. For a compact based space $Y$ we let $C(Y)$ denote the $C^{*}$-algebra of continuous $\mathbb{C}$-valued functions which vanish on the base point. Then by the equivalence between homotopy theoretic and analytic $K$-homology [13] we have an isomorphism

$$
\pi_{n+2}\left(K \wedge B_{+} \wedge C_{l}\right) \cong K K_{n+2}\left(C\left(B_{+} \wedge C_{l}\right), \mathbb{C}\right)
$$

The Spin ${ }^{c}$-extension of the Levi-Civita connection on $W$ together with the standard $S p i n^{c}$-geometry of $S^{1}$ induce a corresponding product $S p i n^{c}$-extension of the Levi-Civita connection on $S^{1} \times W$. The $S p i n^{c}$-geometry on $S^{1}$ also induces such a geometry on the boundary $\partial S_{l}^{2} \cong l S^{1}$ which we extend to $S_{l}^{2}$, again with a product structure. We get a corresponding product Spin $^{c}$-extension of the Levi-Civita connection on $S_{l}^{2} \times M$. These geometric structures glue nicely and give a $\operatorname{Spin}^{c}$-extension of the Levi-Civita connection on $\tilde{W}$. We let $D_{\tilde{W}}$ denote the corresponding Dirac operator. It acts on the complex spinor bundle $S(T \tilde{W})$. The Hilbert space $L^{2}(\tilde{W}, S(\tilde{W}))$ of square integrable sections of this bundle carries an action $\rho$ of the $C^{*}$-algebra $C\left(\tilde{W}_{+}\right)$of continuous functions on $\tilde{W}$ by multiplication. The triple

$$
\left(\not D_{\tilde{W}}\right):=\left(L^{2}(\tilde{W}, S(\tilde{W})), \not D_{\tilde{W}}, \rho\right)
$$

is an unbounded Kasparov module for the pair of $C^{*}$-algebras $\left(C\left(\tilde{W}_{+}\right), \mathbb{C}\right)$ and represents a class

$$
\left[\not D_{\tilde{W}}\right] \in K K_{n+2}\left(C\left(\tilde{W}_{+}\right), \mathbb{C}\right) .
$$

The map $(\tilde{F}, \tilde{G})$ induces a homomorphism of $C^{*}$-algebras

$$
(\tilde{F}, \tilde{G})^{*}: C\left(B_{+} \wedge C_{l}\right) \rightarrow C\left(\tilde{W}_{+}\right)
$$

which in turn induces the push-forward in analytic $K$-homology in the statement of the following Lemma. 
Lemma 3.8 The image of the class $\hat{x} \in \pi_{n+2}\left(M B \wedge C_{l}\right)$ under the composition of the unit (41), Thom isomorphism, (42) and the identification (43) is given by

$$
(\tilde{F}, \tilde{G})_{*}\left[D_{\tilde{W}}\right] \in K K_{n+2}\left(C\left(B_{+} \wedge C_{l}\right), \mathbb{C}\right) .
$$

Proof. The image of $\hat{x}$ under the unit and Thom isomorphism is given

$$
\operatorname{Thom}_{K}(\epsilon(\hat{x}))=\beta(\Delta(\hat{x})) \in \pi_{n+2}\left(K \wedge B_{+} \wedge C_{l}\right),
$$

where $\Delta: M B \rightarrow M B \wedge B_{+}$is the Thom diagonal and $\beta: M B \rightarrow K$ is the $K$-orientation of $M B$ given by 111$)$. We have $\Delta(\hat{x})=\iota[\tilde{W},(\tilde{F},(\tilde{F}, \tilde{G}))] \in \pi_{n+2}\left(M B \wedge B_{+} \wedge C_{l}\right)$. Formally we can view this as the push-forward of the $B$-bordism fundamental class of $W$ along the map $(\tilde{F}, \tilde{G})$. Its image under the $K$-orientation $\beta$ is then the push-forward of the $K$ theory fundamental class of $\tilde{W}$ associated to the Spin $^{c}$-structure along this map. In the analytic picture of $K$-homology the $K$-theory fundamental class of $\tilde{W}$ is represented by the $\operatorname{Spin}^{c}$-Dirac operator. Hence it is equal to $\left[D_{\tilde{W}}\right]$. We thus get

$$
\beta(\iota[\tilde{W},(\tilde{F},(\tilde{F}, \tilde{G}))])=(\tilde{F}, \tilde{G})_{*}\left[\not D_{\tilde{W}}\right] .
$$

We let $\phi \in K^{0}(B)$. The pairing on the left-hand side in the following calculation in $\pi_{n+2}\left(K \wedge C_{l}\right) \cong \mathbb{Z} / l \mathbb{Z}$ is reminiscent to the evaluation occurring in the definition of $\eta^{\text {top }}$ :

$$
\begin{aligned}
& \left\langle\operatorname{Thom}^{K}(\phi), \epsilon(\hat{x})\right\rangle=\left\langle\phi, \operatorname{Thom}_{K}(\epsilon(\hat{x}))\right\rangle \\
& \stackrel{\text { Lemma }}{=}\left\langle\phi .8,(\tilde{F}, \tilde{G})_{*}\left[\not D_{\tilde{W}}\right]\right\rangle \\
& =\tilde{G}_{*}\left(\left[\not D_{\tilde{W}}\right] \cap \tilde{F}^{*} \phi\right) .
\end{aligned}
$$

We choose a geometric bundle $\tilde{\mathbf{V}}$ whose underlying $K$-theory class is equal to $\tilde{F}^{*} \phi$. The restriction of the map $\tilde{F}$ to the part $S^{1} \times W \subset \tilde{W}$ factors over the projection to $W$ and $F: W \rightarrow B$. Hence we can assume that the restriction of $\tilde{\mathbf{V}}$ to $S^{1} \times W \subset \tilde{W}$ is isomorphic to the pull-back of the bundle $\mathbf{U}$ on $W$, if we allow some stabilisation of $\tilde{\mathbf{V}}$ and $\mathbf{U}$. In the $K K$-picture the $\cap$-product

$$
\left[\not D_{\tilde{W}}\right] \cap \tilde{F}^{*} \phi \in K K_{n+2}\left(C\left(\tilde{W}_{+}\right), \mathbb{C}\right)
$$

is realised by the unbounded Kasparov module $\left(L^{2}(\tilde{W}, S(\tilde{W}) \otimes \tilde{\mathbf{V}}), \not D_{\tilde{W}} \otimes \tilde{\mathbf{V}}, \rho\right)$ associated to the twisted Dirac operator $\not_{\tilde{W}} \otimes \tilde{\mathbf{V}}$, where $\rho$ again denotes the action of $C\left(\tilde{W}_{+}\right)$on $L^{2}(\tilde{W}, S(\tilde{W}) \otimes \tilde{\mathbf{V}})$ by multiplication. Hence we have

$$
\left[\not D_{\tilde{W}} \otimes \tilde{\mathbf{V}}\right]=\left[D_{\tilde{W}}\right] \cap(\tilde{F}, \tilde{G})^{*} \phi
$$

We conclude that $\eta^{t o p}(x) \in Q_{n}(M B)$ is represented by the map

$$
K^{0}(B) \ni \phi \mapsto \tilde{G}_{*}\left[D_{\tilde{W}} \otimes \tilde{\mathbf{V}}\right] \in K K_{n+2}\left(C\left(C_{l}\right), \mathbb{C}\right) \cong \pi_{n+1}(K \mathbb{Z} / l \mathbb{Z}) \subset \pi_{n+1}(K \mathbb{Q} / \mathbb{Z})
$$


where the last inclusion is induced by 35 .

Next we want to calculate the element in $\mathbb{Z} / l \mathbb{Z}$ given by $\tilde{G}_{*}\left(\left[D_{\tilde{W}} \otimes \tilde{\mathbf{V}}\right]\right)$. Since the usual index theorem [9] calculates integral indices we have to construct and calculate an integral representative of this $\mathbb{Z} / l \mathbb{Z}$-valued index. The inclusion of the cone base $i: S^{1} \rightarrow C_{l}$ induces a surjective map

$$
\mathbb{Z} \cong \pi_{n+2}\left(K \wedge S^{1}\right) \rightarrow \pi_{n+2}\left(K \wedge C_{l}\right) \cong \mathbb{Z} / l \mathbb{Z}
$$

We try to construct a lift of $\tilde{G}_{*}\left(\left[D_{\tilde{W}} \otimes \tilde{\mathbf{V}}\right]\right)$ to $\pi_{n+2}\left(K \wedge S^{1}\right)$ by providing a factorisation $\gamma$ as in the diagram

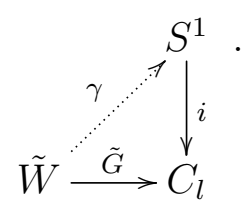

For our given representative such a factorisation does not exist in general. The idea is to modify the representative without changing its $\mathbb{Z} / l \mathbb{Z}$-valued index such that this lift exists for the modified cycle.

Note that $M$ is a closed odd-dimensional manifold. The Dirac operator $D_{M} \otimes \mathbf{V}$ is selfadjoint. We can find a selfadjoint smoothing operator $Q$ on $L^{2}(M, S(M) \otimes V)$ such that $\not_{M} \otimes \mathbf{V}+Q$ is invertible. In [20] such a perturbation was called a taming. As described in this reference a taming can be lifted to the product $S_{l}^{2} \times M$ and also to a collar neighbourhood $l\left(S^{1} \times(-\epsilon, 0] \times M\right) \cong Z \subset S^{1} \times W$ of $\partial\left(S^{1} \times W\right)$. This lift is a selfadjoint operator $\bar{Q}$ on $L^{2}\left(Z \cup_{l\left(S^{1} \times M\right)} S_{l}^{2} \times M, S(\tilde{W}) \otimes \tilde{V}\right)$ which is an integral operator along $M$ and local in the remaining directions. Let $\chi: \tilde{W} \rightarrow[0,1]$ be a cut-off function which is supported on $Z \cup_{l\left(S^{1} \times M\right)} S_{l}^{2} \times M$, is equal to one in a neighbourhood of the subset $S_{l}^{2} \times M$, and only depends on the normal variable near $\partial\left(S^{1} \times W\right)$. We define the extension $\tilde{Q}:=\chi \bar{Q} \chi$ of $\bar{Q}$ to all of $\tilde{W}$. Note that $\tilde{Q}$ commutes with the image of $\tilde{G}^{*}\left(C\left(C_{l}\right)\right)$. Adding $\tilde{Q}$ to $\not_{W} \otimes \tilde{\mathbf{V}}$ gives a relatively compact perturbation. Therefore we have

$$
\tilde{G}_{*}\left[\not D_{\tilde{W}} \otimes \tilde{\mathbf{V}}\right]=\tilde{G}_{*}\left[\not D_{\tilde{W}} \otimes \tilde{\mathbf{V}}+\tilde{Q}\right]
$$

in $K K_{n+2}\left(C\left(C_{l}\right), \mathbb{C}\right)$. On the part $S_{l}^{2} \times M \subset \tilde{W}$ the perturbed operator $\not D_{W} \otimes \tilde{\mathbf{V}}+\tilde{Q}$ is invertible along the fibres of the projection to $S_{l}^{2}$.

On $S^{1} \times(0, \infty)$ we fix a warped product Riemannian metric of the form $g:=d t^{2}+f(t) g^{T S^{1}}$, where $f_{\mid[0,1]} \equiv 1$ and $f(t)=t$ for $t \in(2, \infty)$. The precise form is not important, but we need that $\lim _{t \rightarrow \infty} f(t)=\infty$. We further choose a $S p i n^{c}$-extension of this geometry. We define the manifold

$$
\tilde{\tilde{W}}:=S^{1} \times W \cup_{l\left(S^{1} \times M\right)} l\left(S^{1} \times[0, \infty) \times M\right) .
$$

Its Spin $^{c}$-geometry is given as the product of the geometries of $W$ and $S^{1}$ on the lefthand side, and by the product of the geometries on $l M$ and $S^{1} \times(0, \infty)$ on the right-hand side. In a similar manner we define the geometric bundle $\tilde{\tilde{\mathbf{V}}}$ on $\tilde{W}$ by a cylindrical extension of $\tilde{\mathbf{V}}_{\mid S^{1} \times W}$. We define an operator $\tilde{\tilde{Q}}$ similarly to $\tilde{Q}$ by lifting $Q$ to the cylinder 
$S^{1} \times[0, \infty) \times M$ and cutting off in the interior of $S^{1} \times W$. Finally we let $\tilde{\tilde{G}}: \tilde{\tilde{W}} \rightarrow C_{l}$ be given by $G$ on $S^{1} \times W$ and the radially constant extension of $(\tilde{G})_{\mid \partial\left(S^{1} \times W\right)}$ to the cylinder $l\left([0, \infty) \times S^{1} \times M\right)$.

To every complete Riemannian manifold $(N, g)$ we associate the commutative $C^{*}$-algebra $C_{g}(N)$ defined as the closure in the sup-norm of the algebra $C_{g}^{\infty}(N)$ of all bounded smooth functions $f$ on $N$ such that $|d f| \in C_{0}(N)$. Note that if $N$ is compact, then $C_{g}(N)=C(N)$. The operator $D_{\tilde{W}} \otimes \tilde{\tilde{\mathbf{V}}}+\tilde{\tilde{Q}}$ is invertible along the fibre $M$ of the projection from the cylindrical end of $\tilde{\tilde{W}}$ to $S^{1} \times[0, \infty)$. Therefore it is invertible at infinity in the sense of [19, Assumption 1]. The arguments given [19, Section 1] show that $\left(L^{2}(\tilde{\tilde{W}}, S(T \tilde{\tilde{W}}) \otimes\right.$ $\left.\tilde{\tilde{V}}), D_{\tilde{\tilde{W}}} \otimes \tilde{\tilde{\mathbf{V}}}+\tilde{\tilde{Q}}, \tilde{\tilde{\rho}}\right)$ is an unbounded Kasparov module over $C_{g}(\tilde{\tilde{W}})$. We write $\left[D_{\tilde{\tilde{W}}} \otimes\right.$ $\tilde{\tilde{\mathbf{V}}}+\tilde{\tilde{Q}}] \in K K_{n+2}\left(C_{g}(\tilde{\tilde{W}}), \mathbb{C}\right)$ for its class. Because of the choice of the warped product metric we have the homomorphism of $C^{*}$-algebras $\tilde{\tilde{G}}^{*}: C\left(C_{l}\right) \rightarrow C_{g}(\tilde{\tilde{W}})$ so that the class $\tilde{\tilde{G}}_{*}\left[D_{\tilde{\tilde{W}}} \otimes \tilde{\tilde{\mathbf{V}}}+\tilde{\tilde{Q}}\right] \in K K_{n+2}\left(C\left(C_{l}\right), \mathbb{C}\right)$ is well-defined.

The operators $\not_{W} \otimes \tilde{\mathbf{V}}+\tilde{Q}$ and $\not_{\tilde{W}} \otimes \tilde{\tilde{\mathbf{V}}}+\tilde{\tilde{Q}}$ coincide on $S^{1} \times W$ and are invertible along the fibres $M$ outside of this submanifold of $\tilde{W}$ and $\tilde{\tilde{W}}$.

We let $N_{1}$ be the double of $l\left(M \times S^{1} \times[0, \infty)\right)$ which carries an obvious geometric bundle $\mathbf{V}_{1}$, taming $Q_{1}$, and admits a map $G_{1}: N_{1} \rightarrow C_{l}$. The associated $K$-theory class $\left[N_{1}\right]:=$ $\left[\not D_{N_{1}} \otimes \mathbf{V}_{1}+Q_{1}\right] \in K K_{n+2}\left(C_{g}\left(N_{1}\right), \mathbb{C}\right)$ vanishes since $\not_{N_{1}} \otimes \mathbf{V}_{1}+Q_{1}$ is invertible. Similarly we define $N_{2}$, a map $G_{2}: N_{2} \rightarrow C_{l}$, and a trivial class $\left[\not D_{N} \otimes \mathbf{V}_{2}+Q_{2}\right] \in K K_{n+2}\left(C_{g}\left(N_{2}\right), \mathbb{C}\right)$ by attaching the (reflected) warped product $l M \times S^{1} \times[0, \infty)$ to $M \times S_{l}^{2}$.

In this situation, which is schematically pictured below, we can apply a relative index theorem (the proof of [19, Theorem 1.14] extends since all constructions there are compatible with the action of the $C^{*}$-algebra $\left.C\left(C_{l}\right)\right)$ in order to get the equality! in $K K_{n+2}\left(C\left(C_{l}\right), \mathbb{C}\right)$ :

$$
\tilde{G}_{*}\left[D_{\tilde{W}} \otimes \tilde{\mathbf{V}}\right]=\tilde{G}_{*}\left[D_{\tilde{W}} \otimes \tilde{\mathbf{V}}\right]+G_{1, *}\left[N_{1}\right] \stackrel{!}{=} \tilde{\tilde{G}}_{*}\left[D_{\tilde{W}} \otimes \tilde{\tilde{\mathbf{V}}}+\tilde{\tilde{Q}}\right]+G_{2, *}\left[N_{2}\right]=\tilde{\tilde{G}}_{*}\left[D_{\tilde{W}} \otimes \tilde{\tilde{\mathbf{V}}}+\tilde{\tilde{Q}}\right] .
$$
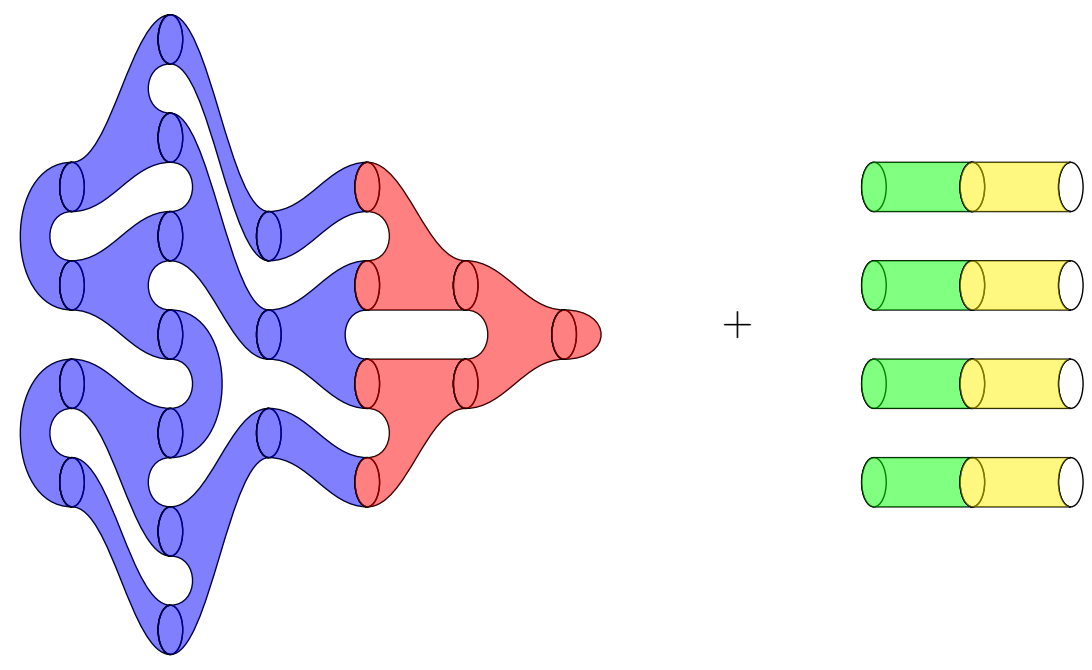


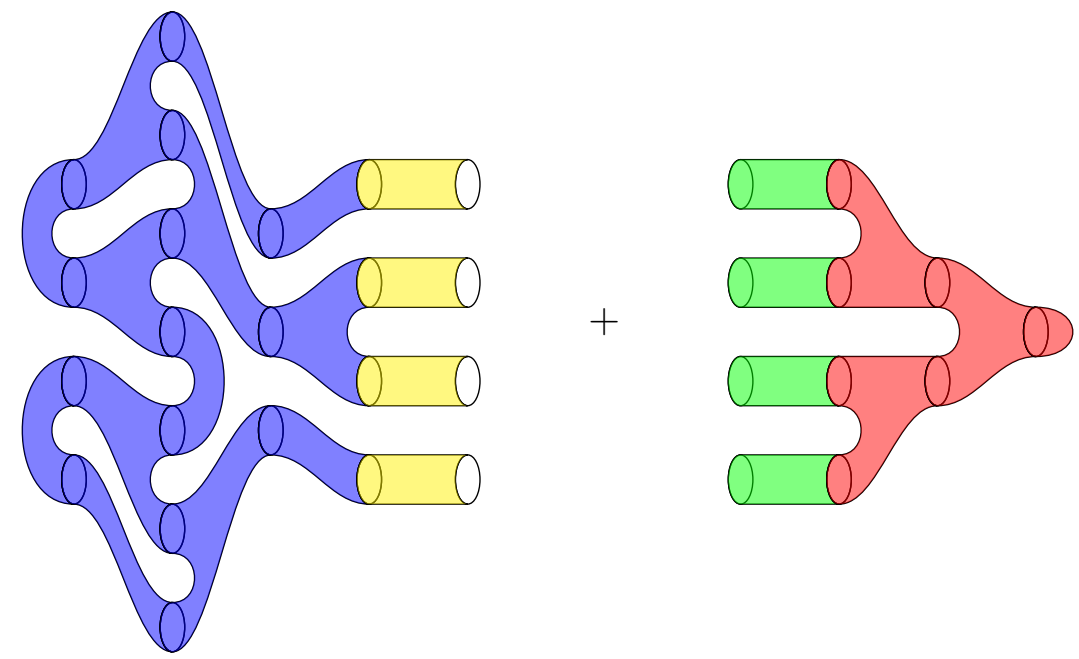

A picture of the relative index theorem. The operator is invertible on the parts which are not blue. The index of the operator associated to the upper picture is the index of its left part $\tilde{W}$. The index is preserved under cut-and paste as indicated. The index of the operator associated to the lower picture is again the index of the left part $\tilde{\tilde{W}}$.

Note the factorisation $\tilde{\tilde{G}}: \tilde{\tilde{W}} \stackrel{\mathrm{pr}^{1}}{\rightarrow} S^{1} \stackrel{i}{\rightarrow} C_{l}$, where the last map is the embedding of the cone basis. Therefore

$$
\operatorname{pr}_{S^{1} *}\left[\not D_{\tilde{\tilde{W}}} \otimes \tilde{\tilde{\mathbf{V}}}+\tilde{\tilde{Q}}\right] \in K K_{n+2}\left(C\left(S^{1}\right), \mathbb{C}\right) \cong \pi_{n+2}\left(K \wedge S^{1}\right) \cong \mathbb{Z}
$$

represents the desired integral lift. We reduce the calculation of this integer to a calculation of a Fredholm index by suspending once more. Let $\mathbf{L}$ be a geometric line bundle on $S^{1} \times S^{1}$ such that $c_{1}(L) \in H^{2}\left(S^{1} \times S^{1} ; \mathbb{Z}\right)$ is a generator and the sign is fixed such that

$$
\operatorname{index}\left(\not_{S^{1} \times S^{1}} \otimes \mathbf{L}\right)=1 \text {. }
$$

We define $\tilde{\tilde{\tilde{W}}}:=S^{1} \times \tilde{\tilde{W}}$ with the product $S p i n^{c}$-geometry. Then the desired integer 45 is the index of the Fredholm operator $\not_{\tilde{\tilde{W}}} \otimes \operatorname{pr}_{\tilde{W}} \tilde{\tilde{\mathbf{V}}} \otimes \operatorname{pr}_{S^{1} \times S^{1}}^{*} \mathbf{L}+\tilde{\tilde{\tilde{Q}}}$ where $\tilde{\tilde{\tilde{Q}}}$ is the taming induced by $\tilde{\tilde{Q}}$. In order to see this note that the identification $K K_{1}\left(C\left(S^{1}\right), \mathbb{C}\right) \stackrel{\cong}{\rightarrow}$ is given by the iterated Kasparov product $x \mapsto[\mathbf{L}] \otimes_{C\left(S^{1} \times S^{1}\right)}\left(\left[D_{S^{1}}\right] \otimes_{\mathbb{C}} x\right) \in K K_{2}(\mathbb{C}, \mathbb{C}) \cong \mathbb{Z}$, where $\left[D_{S^{1}}\right] \in K K_{1}\left(C\left(S^{1}\right), \mathbb{C}\right)$ is the class of the standard $S p i n^{c}$-Dirac operator on $S^{1}$ and $[\mathbf{L}] \in K K_{0}\left(\mathbb{C}, C\left(S^{1} \times S^{1}\right)\right)$ is the class represented by the line bundle $\mathbf{L}$. We now have

$$
\operatorname{pr}_{S^{1} \times S^{1} *}\left[D_{\tilde{\tilde{W}}} \otimes \operatorname{pr}_{\tilde{W}} \tilde{\tilde{\mathbf{V}}}+\tilde{\tilde{Q}}\right]=\left[D_{S^{1}}\right] \otimes \mathbb{C} \operatorname{pr}_{S^{1} *}\left[D_{\tilde{\tilde{W}}} \otimes \tilde{\tilde{\mathbf{V}}}+\tilde{\tilde{Q}}\right]
$$

in $K K_{2}\left(C\left(S^{1} \times S^{1}\right), \mathbb{C}\right)$ and therefore, using the relation of twisting Dirac operators and Kasparov products,

$$
\left[\not D_{\tilde{\tilde{W}}} \otimes \operatorname{pr}_{\tilde{\tilde{W}}} \tilde{\tilde{\mathbf{V}}} \otimes \operatorname{pr}_{S^{1} \times S^{1}}^{*} \mathbf{L}+\tilde{\tilde{\tilde{Q}}}\right]=[\mathbf{L}] \otimes_{C\left(S^{1} \times S^{1}\right)} \operatorname{pr}_{S^{1} \times S^{1} *}\left[D_{\tilde{\tilde{W}}} \otimes \operatorname{pr}_{\tilde{\tilde{W}}} \tilde{\tilde{\mathbf{V}}}+\tilde{\tilde{\tilde{Q}}}\right]
$$


in $K K_{2}(\mathbb{C}, \mathbb{C})$.

We deform the warped product metric on the end of $\tilde{\tilde{W}}$ to a product metric. This produces a continuous family of Fredholm operators and therefore does not change the index. After this deformation we see that $\tilde{\tilde{\tilde{W}}} \cong S^{1} \times S^{1} \times \hat{W}$ geometrically, where $\hat{W}:=W \cup_{\partial W} l([0, \infty) \times M)$ and $\hat{W}$ carries the cylindrical extension of the geometry of $W$. Similarly we let $\hat{\mathbf{U}}$ be the geometric bundle on $\hat{W}$ obtained by the cylindrical extension of $\mathbf{U}$. Then the resulting operator represents the product

$$
\left[\not D_{\hat{W}} \otimes \hat{\mathbf{U}}+\hat{Q}\right] \otimes_{\mathbb{C}}\left[\not D_{S^{1} \times S^{1}} \otimes \mathbf{L}\right]
$$

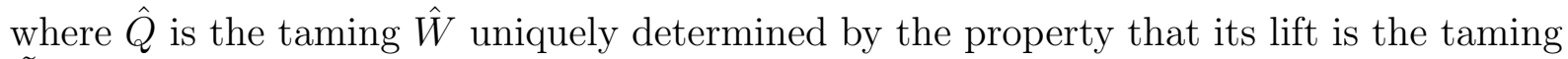
$\tilde{\tilde{Q}}$. Because of (46) the integer (45) is equal to index $\left(D_{\hat{W}} \otimes \hat{\mathbf{U}}+\hat{Q}\right)$.

We now calculate this index. The index theory for these kinds of perturbations of Dirac operators has been developed in [20]. In the language of this reference the operator $Q$ defines a taming $(M \otimes \mathbf{V})_{t}$ of the geometric manifold $M \otimes \mathbf{V}$ and a boundary taming $(W \otimes \mathbf{U})_{b t}$ of the geometric manifold $W \otimes \mathbf{U}$. The following equality holds true by definition of the right-hand side: index $\left(\left(D_{\hat{W}} \otimes \hat{\mathbf{U}}+\hat{Q}\right)=\operatorname{index}\left((W \otimes \mathbf{U})_{b t}\right)\right.$. The index theorem [20, Thm. 4.18] gives

$$
\operatorname{index}\left((W \otimes \mathbf{U})_{b t}\right)=\int_{W} p_{n+1}\left(\mathbf{T d}\left(\tilde{\nabla}^{T W}\right) \wedge \mathbf{c h}\left(\nabla^{U}\right)\right)-\ln \left((M \otimes \mathbf{V})_{t}\right) .
$$

In $\mathbb{R} / \mathbb{Z}$ we have $\left[\eta\left((M \otimes \mathbf{U})_{t}\right)\right]=\xi\left(D_{M} \otimes \mathbf{V}\right)$. Hence by comparison with (28) we get the equality in $\mathbb{Q} / \mathbb{Z}$

$$
\left[\frac{1}{l} \operatorname{index}\left((W \otimes \mathbf{U})_{b t}\right)\right]=\left[\frac{1}{l} \operatorname{index}\left(\left(D_{W} \otimes \mathbf{U}\right)_{A P S}\right)\right]
$$

In view of the construction of $\eta^{a n}$, in particular of $(32)$, we see that the map (44) also represents $\eta^{a n}(x)$. This finishes the proof of Theorem 3.6.

\section{An intrinsic formula}

\subsection{Motivation}

In a typical situation for the theory of the present paper one is given a geometric representative $(M, f)$ for a torsion class $x=[M, f] \in \pi_{n}(M B)$ and wants to calculate the universal $\eta$-invariants $\eta^{t o p}(x)=\eta^{a n}(x) \in Q_{n}(M B)$. The expressions for the universal $\eta$-invariant that we have at our disposal at the moment share the disadvantage that one has to find a lift $\hat{x} \in \pi_{n+1}(M B \mathbb{Q} / \mathbb{Z})$ or a geometric zero bordism $(W, F)$ of $l$ copies of $(M, f)$ explicitly. It is at this point where differential and spectral geometry helps. In the present section we develop a generalisation of Chern-Weyl theory which is designed to finally obtain formulas for the universal $\eta$-invariant which are intrinsic in the cycle $(M, f)$. 
The main new object is the notion of a geometrisation of $(M, f, \tilde{\nabla})$ which is defined in Definition 4.5. It involves differential $K$-theory which is reviewed in Subsection 4.2. In Subsection 4.3 we show the existence of geometrisations and study their functorial properties. In Subsection 4.4 we introduce a special class of geometrisations which we call good. In contrast to general geometrisations they have the property that they extend over zero bordisms. The main result is the intrinsic formula for the universal $\eta$-invariant formulated in Theorem 4.19,

\subsection{Review of differential $K$-theory}

The definition of a geometrisation utilises differential $K$-theory. We refer to [29], [35], [22] for constructions and further information. In the following we review the basic structures which by [23] uniquely characterise differential $K$-theory. Differential $K$-theory is a fivetuple

$$
\left(\hat{K}, I, R, a, \int\right)
$$

of the following objects. The first entry is a contravariant functor

$$
\hat{K}: \text { smooth manifolds } \longrightarrow \mathbb{Z} / 2 \mathbb{Z} \text { - graded commutative rings . }
$$

The remaining entries are natural transformations between functors. The domains and ranges of the first three are given by

$$
\begin{gathered}
I: \hat{K} \rightarrow K, \quad R: \hat{K} \rightarrow \Omega P_{c l} . \\
a: \Omega P / \operatorname{im}(d)[1] \rightarrow \hat{K}
\end{gathered}
$$

Here the evaluation of $\Omega P$ at $M$ is the graded vector space $\Omega P(M):=\Omega(M)\left[b, b^{-1}\right]$ of two-periodic smooth real differential forms on $M$ which carries a differential $d$. By $\Omega P_{c l}(M) \subseteq \Omega P(M)$ we denote its subspace of closed forms. The transformations $R$ and $I$ preserve the ring structures while $a$ is just additive. These transformations are compatible in the sense that for every manifold $M$ the following differential cohomology diagram commutes

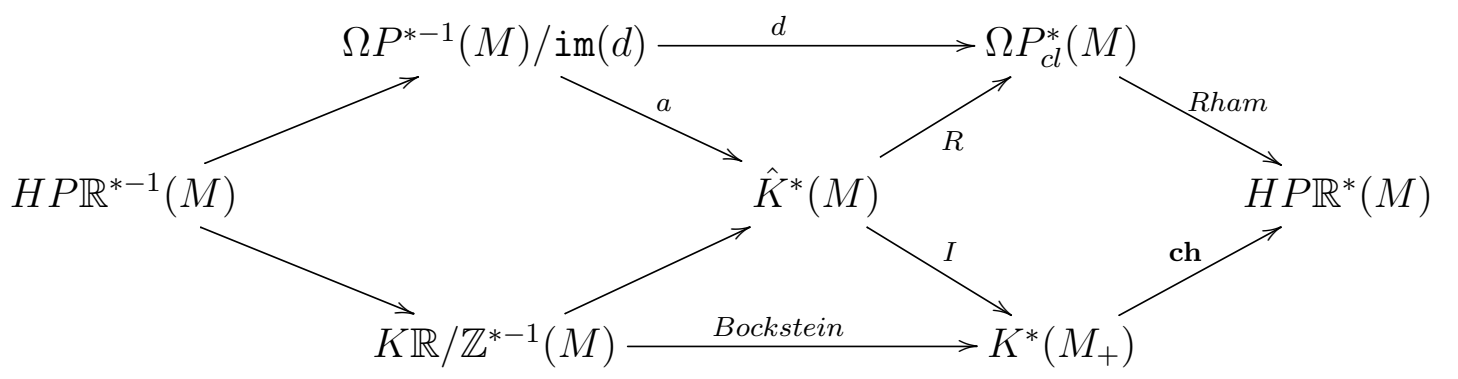

Here we define the spectrum $H P \mathbb{R}$ representing periodic real cohomology similarly as $H P \mathbb{Q}$ in (7). Furthermore, for $\alpha \in \Omega P^{*}(M) / \operatorname{im}(d)$ and $x \in \hat{K}^{*}(M)$ we have the identity

$$
a(\alpha) \cup x=a(\alpha \wedge R(x)) .
$$


The flat part of differential $K$-theory is defined as the kernel of the curvature transformation $R$. It is canonically isomorphic to $\mathbb{R} / \mathbb{Z}-K$-theory (with a shift):

$$
\hat{K}_{\text {flat }}^{*}(M):=\operatorname{ker}\left(R: \hat{K}^{*}(M) \rightarrow \Omega P_{c l}^{*}(M)\right) \cong K \mathbb{R} / \mathbb{Z}^{*-1}(M) .
$$

The sequence

$$
K^{*-1}(M) \stackrel{\text { ch }}{\rightarrow} \Omega P^{*-1}(M) / \mathrm{im}(d) \stackrel{a}{\rightarrow} \hat{K}^{*}(M) \stackrel{I}{\rightarrow} K^{*}(M) \rightarrow 0
$$

is exact. The integration is a natural (in $M$ ) transformation

$$
\int: \hat{K}^{*}\left(S^{1} \times M\right) \rightarrow \hat{K}^{*-1}(M)
$$

whose existence and compatibility with the other structures fixes the odd part of the differential extension uniquely up to unique isomorphism as discussed in [23]. Since we do not need the integration in the present paper we will not write out the long list of these compatibilities explicitly.

Differential $K$-theory is not homotopy invariant. The deviation from homotopy invariance is quantified by the homotopy formula. If $\hat{x} \in \hat{K}^{*}([0,1] \times M)$, then it states that

$$
i_{1}^{*} \hat{x}-i_{0}^{*} \hat{x}=a\left(\int_{[0,1] \times M / M} R(\hat{x})\right) .
$$

Let $\mathbf{V}=\left(V, h^{V}, \nabla^{V}\right)$ be a geometric bundle on manifold $M$, where $h^{V}$ is a hermitean metric which is preserved by the connection $\nabla^{V}$. Then we have a natural class

$$
[\mathbf{V}] \in \hat{K}^{0}(M) .
$$

This class is in fact tautological in the model [22] in view of [22, 2.1.4]. It satisfies

$$
I([\mathbf{V}])=[V] \in K^{0}(M), \quad R([\mathbf{V}])=\operatorname{ch}\left(\nabla^{V}\right) \in \Omega P_{c l}^{0}(M),
$$

where

$$
\operatorname{ch}\left(\nabla^{V}\right):=\operatorname{Tr}\left(\exp \left(-\frac{b R^{\nabla}}{2 \pi i}\right)\right)
$$

is the normalized Chern character form.

Remark 4.1 If we replace $H P \mathbb{R}$ and $\Omega P$ by $H P \mathbb{C}$ and $\Omega P \otimes \mathbb{C}$, then we get a complex version $\hat{K}_{\mathbb{C}}$ of differential $K$-theory with similar properties. A complex vector bundle with connection $\mathbf{V}=\left(V, \nabla^{V}\right)$ on a manifold $M$, where $\nabla^{V}$ is not necessarily hermitean, gives a class $[\mathbf{V}] \in \hat{K}_{\mathbb{C}}^{0}(M)$ such that analogues of the equalities $(52)$ still hold true. For the flat part we get the equivalence

$$
\hat{K}_{\mathbb{C}, \text { flat }}^{0}(M) \cong K \mathbb{C} / \mathbb{Z}^{-1}(M) .
$$




\subsection{Geometrisations}

Let $M$ be a compact manifold equipped with a map $f: M \rightarrow B$. At the moment we do not require any connection of $f$ with the tangent bundle. Nevertheless we must imitate this situation. We can assume that $f$ has a factorisation over $\tilde{f}: M \rightarrow B \operatorname{Spin}^{c}(k)$ as in (22) which classifies a $\operatorname{Spin}^{c}(k)$-bundle $\tilde{f}^{*} Q_{k} \in \operatorname{Spin}^{c}\left(\tilde{f}^{*} \xi_{k}^{\operatorname{Spin}^{c}}\right)$ on $M$. The role of the tangent bundle is taken by the choice of a complementary $S_{p i n}{ }^{c}$-bundle. In detail, we choose an $l$-dimensional oriented euclidean vector bundle $\eta \rightarrow M$ for some $l \geq 0$ together with an orientation preserving isomorphism of euclidean vector bundles.

$$
\eta \oplus \tilde{f}^{*} \xi_{k}^{S p i n^{c}} \cong M \times \mathbb{R}^{l+k}
$$

Then we choose a $\operatorname{Spin}^{c}$-structure $P \in \operatorname{Spin}^{c}(\eta)$ together with an isomorphism

$$
P \otimes \tilde{f}_{k}^{*} Q_{k} \cong Q(l+k)
$$

where we use the isomorphism (53) in order view the left- and right-hand sides in the same groupoid $\operatorname{Spin}^{c}\left(M \times \mathbb{R}^{l+k}\right)$ (see Subsection 3.3 for details).

We choose a connection $\tilde{\nabla}$ on $P$ and get an induced Todd form $\mathbf{T d}(\tilde{\nabla}) \in \Omega P_{c l}^{0}(M)$ which represents the class $f^{*} \mathbf{T} \mathbf{d}^{-1} \in H P \mathbb{Q}^{0}(M)$.

We now consider a continuous homomorpism

$$
\mathcal{G}: K^{0}(B) \rightarrow \hat{K}^{0}(M)
$$

where the domain has the topology described in Remark 2.1 and the target is discrete.

Definition 4.2 A cohomological character for $\mathcal{G}$ is a continuous homomorphism $c_{\mathcal{G}}$ : $H P \mathbb{Q}^{0}(B) \rightarrow \Omega P_{c l}^{0}(M)$ such that the following diagram commutes:

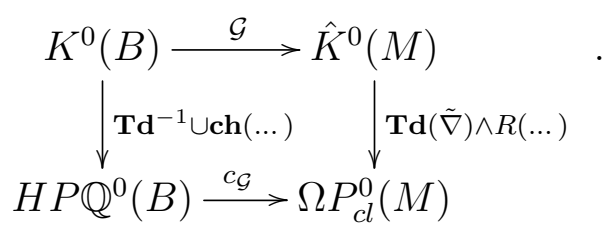

Lemma 4.3 Given $\mathcal{G}$ there exists a cohomological character $c_{\mathcal{G}}$. If $B$ is compact, then it is unique.

Proof. Since $\mathcal{G}$ is continuous there exists a map $r: A \rightarrow B$ from a finite $C W$-complex $A$ such that $\mathcal{G}$ factors over the quotient $K^{0}(B) / \operatorname{ker}\left(r_{K^{0}}^{*}\right)$. For a space $X$ we write

$$
H P \mathbb{Q}^{0}(X)_{0}:=\overline{\operatorname{im}\left(K^{0}(X) \otimes \mathbb{Q}^{\mathbf{T d}^{-1} \cup \mathbf{c h}(\ldots)} \longrightarrow P \mathbb{Q}^{0}(X)\right)} .
$$


We get the diagram

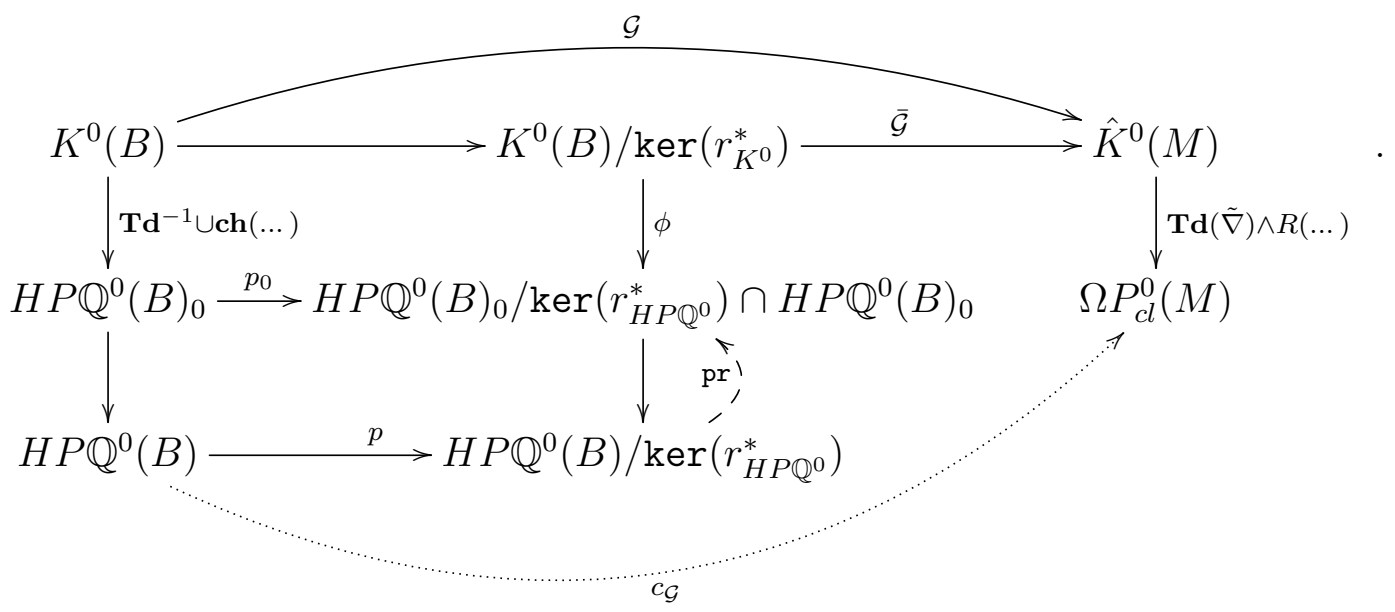

The map $\phi$ induces an isomorphism after tensoring with $\mathbb{Q}$. In order to see this we consider the diagram

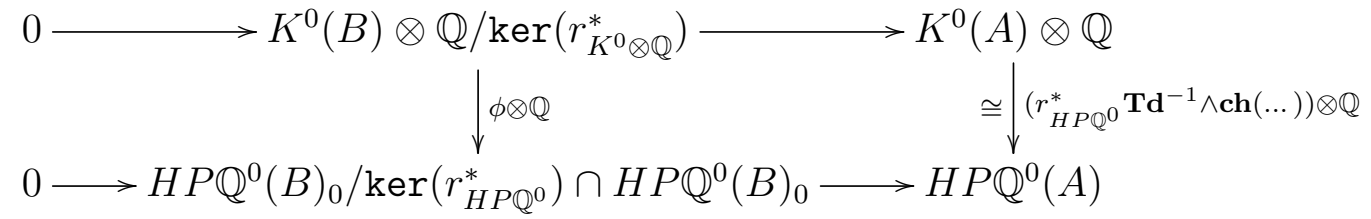

with exact horizontal lines. We immediately see that $\phi \otimes \mathbb{Q}$ is injective. On the other hand, by definition (54) the image of $K^{0}(B) \otimes \mathbb{Q} \stackrel{\left(\mathbf{T d}^{-1} \cup \operatorname{ch}(\ldots)\right) \otimes \mathbb{Q}}{\longrightarrow} H P \mathbb{Q}^{0}(B)$ is dense in $H P \mathbb{Q}^{0}(B)_{0}$. Note that the quotient $H P \mathbb{Q}^{0}(B) / \operatorname{ker}\left(r_{H P \mathbb{Q}^{0}}^{*}\right)$ carries the discrete topology. Therefore the composition

$$
K^{0}(B) \otimes \mathbb{Q} \stackrel{\left(\mathbf{T d}^{-1} \cup \operatorname{ch}(\ldots)\right) \otimes \mathbb{Q}}{\longrightarrow} H P \mathbb{Q}^{0}(B)_{0} \rightarrow H P \mathbb{Q}^{0}(B)_{0} / \operatorname{ker}\left(r_{H P \mathbb{Q}^{0}}^{*}\right) \cap H P \mathbb{Q}^{0}(B)_{0}
$$

is surjective. This implies that $\phi \otimes \mathbb{Q}$ is surjective, too.

After choosing some linear projection

$$
\text { pr : } H P \mathbb{Q}^{0}(B) / \operatorname{ker}\left(r_{H P \mathbb{Q}^{0}}^{*}\right) \rightarrow H P \mathbb{Q}^{0}(B)_{0} / \operatorname{ker}\left(r_{H P \mathbb{Q}^{0}}^{*}\right) \cap H P \mathbb{Q}^{0}(B)_{0}
$$

we can define a cohomological character by

$$
c_{\mathcal{G}}(x):=\mathbf{T} \mathbf{d}(\tilde{\nabla}) \wedge((R \circ \overline{\mathcal{G}}) \otimes \mathbb{Q}) \circ(\phi \otimes \mathbb{Q})^{-1}(\operatorname{pr}(p(x))), \quad x \in H P \mathbb{Q}^{0}(B) .
$$

Since it factorizes over $p$ it is continuous.

If $B$ is compact, then $H P \mathbb{Q}^{0}(B)_{0}=H P \mathbb{Q}^{0}(B)$ and this implies uniqueness of $c_{\mathcal{G}}$. 
Example 4.4 (Völkl) If $B$ is not compact, then a cohomological character is not necessarily unique. Consider $B:=K(\mathbb{Z}, 4)$ and assume that $M$ is compact. By [4, Thm. II] the reduced $K$-theory group $\tilde{K}^{0}(B)$ consists of phantom classes. Consequently, a continuous map $\mathcal{G}: \tilde{K}^{0}(B) \rightarrow K^{0}(M)$ must be trivial. Furthermore, we have $H P \mathbb{Q}(B)_{0}=b^{0} \mathbb{Q}$. Let $\mathcal{G}:=0$. In this case any continuous homomorphisms $H P \mathbb{Q}^{0}(B) \rightarrow \Omega P_{c l}^{0}(M)$ vanishing on $b^{0} \mathbb{Q}$ can serve as a cohomological character $c_{\mathcal{G}}$. Note that $H P \mathbb{Q}^{0}(B) \cong \mathbb{Q}[[q]]$ with $q:=b^{2} u$ for the canonical class $u \in H^{4}(K(\mathbb{Z}, 4), \mathbb{Q})$, so that there are many such homomorphisms.

We say that the cohomological character $c_{\mathcal{G}}$ preserves degree if it preserves the decompositions

$$
H P \mathbb{Q}^{0}(B) \cong \prod_{k \in \mathbb{Z}} b^{-k} H^{2 k}(B ; \mathbb{Q}), \quad \Omega P_{c l}^{0}(M) \cong \prod_{k \in \mathbb{Z}} b^{-k} \Omega_{c l}^{2 k}(M)
$$

Definition 4.5 A geometrisation of $(M, f, \tilde{\nabla})$ is a continuous homomorphism

$$
\mathcal{G}: K^{0}(B) \rightarrow \hat{K}^{0}(M)
$$

such that the following diagram

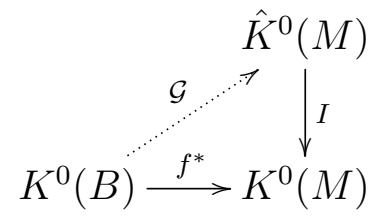

commutes, and which admits a degree-preserving cohomological character.

Note that the notion of a geometrisation only depends on the form $\mathbf{T d}(\tilde{\nabla})$ representing the class $\mathbf{T} \mathbf{d}^{-1}$. The additional rigidity of the construction related with the choice of $\eta, \tilde{f}$ and the isomorphism (53) is only used for the construction of the pull-back of geometrisations below.

Example 4.6 The notion of a geometrisation generalises the notion of a connection. This is demonstrated in Lemma 5.13 for the case $B=B$ Spin. At this place we will discuss another example where we put $B:=B \operatorname{Spin}^{c} \times B \Gamma$ for some compact Lie group $\Gamma$ and $B \rightarrow B \operatorname{Spin}^{c}$ is the projection. We write maps to $B \operatorname{Spin}^{c} \times B \Gamma$ as pairs $(f, g)$.

Let us assume that we already have a geometrisation $\mathcal{G}^{0}$ of $(M, f, \tilde{\nabla})$ with a degreepreserving cohomological character $c_{\mathcal{G}^{0}}$. Its existence is guaranteed by Proposition 4.8. The map $g: M \rightarrow B \Gamma$ classifies a $\Gamma$-principal bundle $R \rightarrow M$. We choose a connection $\nabla^{R}$ on $R$.

Lemma 4.7 There exists a natural geometrisation $\mathcal{G}$ of $(M,(f, g), \tilde{\nabla})$ associated to this data. 
Proof. The completion theorem [8] gives an isomorphism $K^{0}(B \Gamma) \cong R(\Gamma)_{I_{\Gamma}}$ of topological groups, where $I_{\Gamma} \subseteq R(\Gamma)$ is the dimension-ideal of the integral representation ring. We consider a representation $\sigma: \Gamma \rightarrow U\left(m_{\sigma}\right)$ which represents an element $[\sigma] \in K^{0}(B \Gamma)$. The associated complex vector bundle $V_{\sigma}:=R \times_{\Gamma, \sigma} \mathbb{C}^{m_{\sigma}}$ on $M$ then represents the element $\left[V_{\sigma}\right]=f^{*}[\sigma] \in K^{0}(M)$. This bundle comes with a hermitean metric $h^{V_{\sigma}}$ and a metric connection $\nabla^{V_{\sigma}}$ induced by $\nabla^{R}$. We therefore get a geometric bundle $\mathbf{V}_{\sigma}:=\left(V_{\sigma}, \nabla^{V_{\sigma}}, \nabla^{V_{\sigma}}\right)$. It represents the class $\left[\mathbf{V}_{\sigma}\right] \in \hat{K}^{0}(M)$ such that $\left[V_{\sigma}\right]=I\left(\left[\mathbf{V}_{\sigma}\right]\right)$ in $K^{0}(M)$, see (51). Let $\phi \in K^{0}\left(B \operatorname{Spin}^{c}\right)$. Then we get the element $\phi \times[\sigma] \in K^{0}\left(B \operatorname{Spin}^{c} \times B \Gamma\right)=K^{0}(B)$. We define

$$
\mathcal{G}(\phi \times[\sigma]):=\mathcal{G}^{0}(\phi) \cup\left[\mathbf{V}_{\sigma}\right] .
$$

By linear extension this construction defines the map $\mathcal{G}$ on a dense subgroup of $K^{0}(B)$. We now show that the map $\mathcal{G}$ extends by continuity to all of $K^{0}(B)$ and defines a geometrisation of $(M,(f, g), \tilde{\nabla})$. Indeed, the map $R(\Gamma) \rightarrow \hat{K}^{0}(M)$ induced by $\sigma \mapsto\left[\mathbf{V}_{\sigma}\right]$ is multiplicative and annihilates $I_{\Gamma}^{2 n+1}$, where $n:=\operatorname{dim}(M)$. Therefore, since $\mathcal{G}^{0}$ is continuous, the map $\mathcal{G}$ is continuous as well. We now use the fact that $H P \mathbb{Q}^{0}(B \Gamma)$ is topologically generated by the classes $\operatorname{ch}([\sigma])$ for $\sigma \in R(\Gamma)$. We let $c_{\Gamma}: H P \mathbb{Q}^{0}(B \Gamma) \rightarrow \Omega P_{c l}^{0}(M)$ be the unique continuous map such that $\operatorname{ch}\left(\nabla^{V_{\sigma}}\right)=c_{\Gamma}(\operatorname{ch}([\sigma]))$. Note that $c_{\Gamma}$ preserves degree. Since the cohomological character $c_{\mathcal{G}^{0}}$ preserves degree, we can take $c_{\mathcal{G}}:=c_{\mathcal{G}^{0}} \wedge c_{\Gamma}$ of $\mathcal{G}$ as a degree-preserving cohomological character for $\mathcal{G}$.

The geometrisation $\mathcal{G}$ allows to recover the Chern character form of $\nabla^{V_{\sigma}}$ by

$$
\operatorname{ch}\left(\nabla^{V_{\sigma}}\right)=\mathbf{T d}(\tilde{\nabla})^{-1} \wedge R(\mathcal{G}(1 \otimes[\sigma])) .
$$

It also allows to partially recover transgressions as we will explain in the following. If $\nabla^{R \prime}$ is a second connection on $R$ and $\mathcal{G}^{\prime}$ is the associated geometrisation, then

$$
\mathcal{G}^{\prime}(1 \otimes[\sigma])-\mathcal{G}_{M}^{\prime}(1 \otimes[\sigma])=a\left(\mathbf{T d}(\tilde{\nabla}) \wedge \tilde{\mathbf{c h}}\left(\nabla^{V_{\sigma^{\prime}}}, \nabla^{V_{\sigma}}\right)\right) .
$$

Here $\tilde{\mathbf{c h}}\left(\nabla^{V_{\sigma^{\prime}}}, \nabla^{V_{\sigma}}\right) \in \Omega P^{-1}(M)$ denotes the transgression form which satisfies

$$
d \tilde{\operatorname{ch}}\left(\nabla^{V_{\sigma}}, \nabla^{V_{\sigma}}\right)=\operatorname{ch}\left(\nabla^{V^{\prime}}\right)-\operatorname{ch}\left(\nabla^{V_{\sigma}}\right) .
$$

[End of Example 4.6]

The following Proposition 4.8 asserts that geometrisations exist. Its proof uses the functoriality of geometrisations in the space $B$. Consider a map $\phi$ over $B S_{p i n}^{c}$, i.e. a homotopy commutative diagram

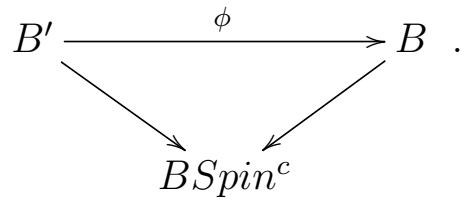

Given a geometrisation $\mathcal{G}$ of $(M, \phi \circ f, \tilde{\nabla})$ we get a geometrisation

$$
\phi_{*} \mathcal{G}:=\mathcal{G} \circ \phi^{*}
$$


of $(M, f, \tilde{\nabla})$.

Note that our standing assumption is that $M$ is compact.

Proposition 4.8 Given $(M, f, \tilde{\nabla})$ there exists a geometrisation.

Proof. Since $M$ is compact the map $f$ factors over a compact subspace of $B$. In view of the functoriality of the geometrisation (55) we can assume that $B$ is compact. Then $K^{0}(B)$ is a finitely generated abelian group. We choose a decomposition

$$
K^{0}(B) \cong A_{\text {tors }} \oplus A_{\text {free }}
$$

into a torsion and a free part. We write

$$
A_{\text {tors }}:=\bigoplus_{y \in I} y \mathbb{Z} / \operatorname{ord}(y) \mathbb{Z}
$$

for some set of generators $I \subset A_{\text {tors }}$. For all $y \in I$, using the exactness at the right end of 49 , we choose $\tilde{y}_{0} \in \hat{K}^{0}(M)$ such that $I\left(\tilde{y}_{0}\right)=f^{*} y$. Then $\operatorname{ord}(y) \tilde{y}_{0}=a\left(\omega_{y}\right)$ for some $\omega_{y} \in \Omega P^{-1}(M) / \operatorname{im}(d)$, again by (49). We define

$$
\tilde{y}:=\tilde{y}_{0}-a\left(\frac{1}{\operatorname{ord}(y)} \omega_{y}\right)
$$

Then $\operatorname{ord}(y) \tilde{y}=0$ and we can define $\mathcal{G}_{\mid A_{\text {tors }}}: A_{\text {tors }} \rightarrow \hat{K}^{0}(M)$ such that $\mathcal{G}(y)=\tilde{y}$ for all $y \in I$. Since $\mathbf{T d}^{-1} \wedge \mathbf{c h}$ vanishes on $A_{\text {tors }}$ and $\mathcal{G}_{\mid A_{\text {tors }}}$ maps to flat classes it is clear that the cohomological character of this part of $\mathcal{G}$ preserves degree.

We now come to the free part. We choose a basis $J \subset A_{\text {free }}$ and classes $\tilde{z}_{0} \in \hat{K}^{0}(M)$ such that $I\left(\tilde{z}_{0}\right)=f^{*} z$ for all $z \in J$. We further choose a basis $J^{\prime} \subset A_{\text {free }} \otimes \mathbb{Q}$ such that $\left\{\mathbf{T d}^{-1} \wedge \operatorname{ch}\left(z^{\prime}\right)\right\}_{z^{\prime} \in J^{\prime}}$ is a homogeneous basis with respect to the decomposition

$$
H P \mathbb{Q}^{0}(B) \cong \bigoplus_{m \in \mathbb{Z}} b^{m} H^{2 m}(B ; \mathbb{Q})
$$

We define the even integers $n_{z^{\prime}}:=\operatorname{deg}\left(\mathbf{T d}^{-1} \wedge \operatorname{ch}\left(z^{\prime}\right)\right)$ for all $z^{\prime} \in J^{\prime}$. Then there exists an invertible rational $\left(J, J^{\prime}\right)$-indexed matrix $A$ such that $z=\sum_{z^{\prime} \in J^{\prime}} A_{z z^{\prime}} z^{\prime}$ for all $z \in J$. We now can choose forms $\alpha_{z^{\prime}} \in \Omega P^{-1}(M) / \operatorname{im}(d)$ for all $z^{\prime} \in J^{\prime}$ such that

$$
\sum_{z \in J} A_{z^{\prime} z}^{-1} \operatorname{Td}(\tilde{\nabla}) \wedge R\left(\tilde{z}_{0}\right)-d \alpha_{z^{\prime}} \in b^{\frac{n z^{\prime}}{2}} \Omega_{c l}^{n} z_{z^{\prime}}(M) \subseteq \Omega P_{c l}^{0}(M)
$$

for all $z^{\prime} \in J^{\prime}$. We define

$$
\mathcal{G}_{\mid A_{\text {free }}}: A_{\text {free }} \rightarrow \hat{K}^{0}(M)
$$

by linear extension such that

$$
\mathcal{G}(z)=\tilde{z}_{0}-a\left(\mathbf{T d}(\tilde{\nabla})^{-1} \wedge \sum_{z^{\prime} \in J^{\prime}} A_{z z^{\prime}} \alpha_{z^{\prime}}\right)
$$


By construction its (uniquely determined) cohomological character preserves degree.

Geometrisations can be pulled back along stable Spin $^{c}$-maps over $X$. In detail the construction goes as follows. Let $\left(M^{\prime}, f^{\prime}\right)$ be a compact manifold with a map $f^{\prime}: M^{\prime} \rightarrow B$. We consider a smooth map $h: M^{\prime} \rightarrow M$ such that $f \circ h$ is homotopic to $f^{\prime}$. This implies that we can choose a stable isomorphism of complementary bundles

$$
\eta^{\prime} \oplus\left(M^{\prime} \times \mathbb{R}^{s}\right) \cong h^{*} \eta \oplus\left(M^{\prime} \times \mathbb{R}^{t}\right)
$$

We refine $h$ to a $\operatorname{Spin}^{c}$-map by choosing an isomorphism

$$
P^{\prime} \otimes Q(s) \cong h^{*} P \otimes Q(t)
$$

Assume now that we have connections $\tilde{\nabla}$ on $P$ and $\tilde{\nabla}^{\prime}$ on $P^{\prime}$. They induce connections on the stabilisations $P \otimes Q(t)$ and $P^{\prime} \otimes Q(s)$. We thus can define the transgression

$$
\tilde{\mathbf{T}} \mathbf{d}\left(h^{*} \tilde{\nabla}, \tilde{\nabla}^{\prime}\right) \in \Omega P^{-1}\left(M^{\prime}\right) / \operatorname{im}(d)
$$

where we use the isomorphism $(57)$ in order to compare the stabilisation of $h^{*} \tilde{\nabla}$ with that of $\tilde{\nabla}^{\prime}$ on the same bundle. The transgression satisfies

$$
d \tilde{\mathbf{T}} \mathbf{d}\left(h^{*} \tilde{\nabla}, \tilde{\nabla}^{\prime}\right)=h^{*} \mathbf{T} \mathbf{d}(\tilde{\nabla})-\mathbf{T} \mathbf{d}\left(\tilde{\nabla}^{\prime}\right) .
$$

Let $\mathcal{G}$ be a geometrisation of $(M, f, \tilde{\nabla})$.

Lemma 4.9 If $h: M^{\prime} \rightarrow M$ is a Spin ${ }^{c}$-map between compact manifolds, then there exists a construction of a pull-back $\mathcal{G}^{\prime}:=h^{*} \mathcal{G}$ of $\left(M^{\prime}, f^{\prime}, \tilde{\nabla}^{\prime}\right)$. This pull-back only depends on the joint stable homotopy class of isomorphisms (56) and (57) and is functorial under compositions.

Proof. By our assumptions the equivalence class

$$
\beta:=\tilde{\mathbf{T}} \mathbf{d}\left(h^{*} \tilde{\nabla}, \tilde{\nabla}^{\prime}\right) \wedge \mathbf{T d}\left(\tilde{\nabla}^{\prime}\right)^{-1} \in \Omega P^{-1}\left(M^{\prime}\right) / \operatorname{im}(d)
$$

of forms is well-defined. It satisfies

$$
d \beta=h^{*} \mathbf{T} \mathbf{d}(\tilde{\nabla}) \wedge \mathbf{T} \mathbf{d}\left(\tilde{\nabla}^{\prime}\right)^{-1}-1 .
$$

We define the pull-back $\mathcal{G}^{\prime}:=h^{*} \mathcal{G}$ by

$$
\mathcal{G}^{\prime}(y):=h^{*} \mathcal{G}(y)+a\left(\beta \wedge h^{*} R(\mathcal{G}(y))\right), \quad y \in K^{0}(B)
$$

where $a$ and $R$ belong to the structure maps of differential $K$-theory. We have by construction

$$
\mathbf{T d}\left(\tilde{\nabla}^{\prime}\right) \wedge R\left(\mathcal{G}^{\prime}(y)\right)=h^{*}(\mathbf{T d}(\tilde{\nabla}) \wedge R(\mathcal{G}(y)))
$$


and hence can take $c_{\mathcal{G}^{\prime}}:=h^{*} c_{\mathcal{G}}$ as a cohomological character for $\mathcal{G}^{\prime}$. Since the cohomological character $c_{\mathcal{G}}$ preserves degree, so does the cohomological character of $\mathcal{G}^{\prime}$.

We show that the pull-back is functorial. We consider a second triple $\left(M^{\prime \prime}, f^{\prime \prime}, \tilde{\nabla}^{\prime \prime}\right)$ with a Spin ${ }^{c}$-map $h^{\prime}: M^{\prime \prime} \rightarrow M^{\prime}$ and the associated transgression form $\beta^{\prime}$. Then we have for the iterated pull-back

$$
\begin{aligned}
\mathcal{G}^{\prime \prime}(y) & =h^{\prime *}\left(h^{*}(\mathcal{G}(y))\right)+h^{\prime *}\left(a\left(\beta \wedge h^{*} R(\mathcal{G}(y))\right)\right)+a\left(\beta^{\prime} \wedge h^{\prime *} R\left(\mathcal{G}^{\prime}(y)\right)\right) \\
& =\left(h \circ h^{\prime *}\right)^{*}(\mathcal{G}(y)) \\
+a\left(h^{\prime *} \beta\right. & \wedge h^{\prime *}\left(h^{*}(R(\mathcal{G}(y)))+\beta^{\prime} \wedge h^{\prime *}\left(h^{*} R(\mathcal{G}(y))\right)+\beta^{\prime} \wedge h^{\prime *} d \beta \wedge h^{\prime *}\left(h^{*}(R(\mathcal{G}(y)))\right)\right)
\end{aligned}
$$

Let $\tilde{\beta}$ be the transgression form for the composition $h \circ h^{\prime}$ of $\operatorname{Spin}^{c}$-maps over $X$. Then we must show that

$$
\tilde{\beta}-\left(h^{* *} \beta+\beta^{\prime}+h^{* *} \beta^{\prime} \wedge d \beta\right) \in \operatorname{im}(d)
$$

This follows from

$$
d\left(h^{* *} \beta+\beta^{\prime}+h^{\prime *} \beta^{\prime} \wedge d \beta\right)=h^{* *} h^{*} \mathbf{T} \mathbf{d}(\tilde{\nabla})^{-1} \wedge \mathbf{T} \mathbf{d}\left(\tilde{\nabla}^{\prime \prime}\right)-1=d \tilde{\beta}
$$

and the fact that all these forms are defined by transgressions and the contractibility of the space of connections. The assertion about homotopy invariance easily follows from the homotopy formula (50) for differential $K$-theory.

Note that the form $\beta$ is determined up the closed forms by $(59)$. The refinement of the map $h$ to a $S p i n^{c}$-map is necessary in order to rigidify the choice of $\beta$ up to exact forms by (58) by constructing it via transgression.

Remark 4.10 The identity of $M$ refines to a $\operatorname{Spin}^{c}$-map in a natural way by choosing the identity in (57). The pull-back of geometrisations for the identity of $M$ can be used to transfer a geometrisation defined for one choice of the connection $\tilde{\nabla}$ to a second choice. This allows to define a notion of geometrisation which is independent of the choice of the connection. This could play a role of one wants to classify geometrisations. We will not pursue that goal in the present paper.

\subsection{Good geometrisations}

Assume that $(W, F)$ is a zero-bordism of the $n$-dimensional cycle $(M, f)$. We fix tangential $\operatorname{Spin}^{c}$-structures $P(T W) \in \operatorname{Spin}^{c}(T W)$ and $P(T M) \in \operatorname{Spin}^{c}(T M)$ associated to the normal $S_{p i n}{ }^{c}$-structure on $W$ and $M$, see Definition 3.2. As explained in Subsection 3.3 there is a natural isomorphism of $\operatorname{Spin}^{c}$-structures

$$
P(T M) \otimes Q(1) \cong P(T W)_{\mid M}
$$

which turns the inclusion

$$
i: M \rightarrow W
$$


into a $\operatorname{Spin}^{c}$-map.

We choose a $\operatorname{Spin}^{c}$-extension of the Levi-Civita connection $\tilde{\nabla}^{T W}$ on $W$ with product structure and a Spin ${ }^{c}$-extension of the Levi-Civita connection $\tilde{\nabla}^{T M}$ on $M$ such that the isomorphism (61) preserves the connections. In this situation the form (58) is trivial. Assume now that we have a geometrisation of $\left(W, F, \tilde{\nabla}^{T W}\right)$. Then we can define the restriction $\mathcal{G}_{\partial W}:=\left(\mathcal{G}_{W}\right)_{\mid \partial W}$ as in Lemma 4.9 . It is given by

$$
\mathcal{G}_{\partial W}(\phi)=\mathcal{G}_{W}(\phi)_{\mid \partial W}, \quad \phi \in K^{0}(B)
$$

In general we do not expect that a given geometrisation $\mathcal{G}_{M}$ of $\left(M, f, \tilde{\nabla}^{T M}\right)$ can be obtained by restricting a geometrisation $\mathcal{G}_{W}$ of $\left(W, F, \tilde{\nabla}^{T W}\right)$. In this respect geometrisations are more rigid than connections.

Example 4.11 Here is a very simple example of a geometrisation which does not extend. We consider the case $B=*$ and let $\left(S^{3}, f\right)$ be a cycle for $\pi_{3}(S)$. We choose a normal framing of $S^{3}$ such that it extends over $D^{4}$ so that the framed bordism class $\left[S^{3}, f\right]$ is trivial. Furthermore we equip $S^{3}$ with its standard Riemannian metric.

We let $\mathcal{G}_{0}$ be the good geometrisation of $\left(S^{3}, f, \tilde{\nabla}^{T S^{3}}\right)$ defined in Subsection 5.1. We have $K^{0}(B) \cong K^{0}(*) \cong \mathbb{Z}$ so that a geometrisation is fixed by the image of 1 in $K^{0}\left(S^{3}\right)$. Let $\omega \in \Omega^{3}\left(S^{3}\right)$ be some form. Then we can define a new geometrisation $\mathcal{G}_{\omega}$ of $\left(S^{3}, f, \tilde{\nabla}^{T S^{3}}\right)$ by

$$
\mathcal{G}_{\omega}(1):=\mathcal{G}_{0}(1)+a(\omega) .
$$

It is easy to check, using the fact that $\mathcal{G}_{0}$ does extend by Lemma 4.15 , that $\mathcal{G}_{\omega}$ extends to $D^{4}$ if and only if $\int_{S^{3}} \omega \in \mathbb{Z}$. [End of example 4.11

In order to deal with the problem of non-extendability of geometrisations appropriately we introduce the notion of a $k$-good geometrisation. If $\mathcal{G}_{M}$ is $k$-good with $k \geq \operatorname{dim}(M)+1$, then it will extend to zero bordisms.

For $k \in \mathbb{N}$ we define the notion of $k$-good geometrisations constructively. We consider a map $f_{u}: M_{u} \rightarrow B$ from a smooth compact manifold $M_{u}$, a lift $\tilde{f}_{u}$ together with a choice of a complementary bundle $\eta_{u}$, a connection $\tilde{\nabla}_{u}$ on $P_{u} \in \operatorname{Spin}^{c}\left(\eta_{u}\right)$, and a geometrisation $\mathcal{G}_{u}$ of $\left(M_{u}, f_{u}, \tilde{\nabla}_{u}\right)$. If the map $f: M \rightarrow B$ has a factorisation up to homotopy through a smooth map $h: M \rightarrow M_{u}$, then we can refine $h$ to a stable Spin $^{c}$-map since, after stabilisation, there exists an isomorphism between $h^{*} \eta_{u}$ and $T M$.

Definition 4.12 The geometrisation $\mathcal{G}_{M}$ is called $k$-good if $\mathcal{G}_{M}=h^{*} \mathcal{G}_{u}$ for some choices as above such that $f_{u}$ is $k$-connected. We say that $\mathcal{G}_{M}$ is good, if it is $\operatorname{dim}(M)+1$-good.

Remark 4.13 We consider a sequence $\left(M_{u, i}, f_{u, i}, \tilde{\nabla}^{u, i}\right), i \in \mathbb{N}$, of data as above together with $\operatorname{Spin}^{c}$-maps $h_{u, i}: M_{u, i} \rightarrow M_{u, i+1}$ for all $i \in \mathbb{N}$ such that $f_{u, i+1} \circ h_{u, i} \sim f_{u, i}$ and $f_{u, i}$ is $i$-connected. A family of geometrisations $\mathcal{G}_{u, i}$ of $\left(M_{u, i}, f_{u, i}, \tilde{\nabla}^{u, i}\right)$ for all $i \in \mathbb{N}$ such that $h_{u, i}^{*} \mathcal{G}_{u, i+1}=\mathcal{G}_{u, i}$ will be called a universal geometrisation. Universal geometrisations will be constructed and classified in a forthcoming paper by M.Völkl. 
Let us fix a universal geometrisation. A geometrisation of $\left(M, f, \tilde{\nabla}^{T M}\right)$ will be called very good (relative to the chosen universal geometrisation), if it is isomorphic to $h^{*} \mathcal{G}_{u, i}$ for some $i \in \mathbb{N}$ and $\operatorname{Spin}^{c}$-map $h: M \rightarrow M_{u, i}$ such that $f_{u, i} \circ h \sim f$. Note that such a geometrisation is $\ell$-good for every $\ell \in \mathbb{N}$.

Lemma 4.14 If $B$ has the homotopy type of a $C W$-complex with finite skeleta, then for every triple $\left(M, f, \tilde{\nabla}^{T M}\right)$ and every $k \in \mathbb{N}$ there exists a $k$-good geometrisation.

Proof. By the assumption, for every $k \in \mathbb{N}$ we can find a compact manifold $M_{u}$ and a map $f_{u}: M_{u} \rightarrow B$ such that $f_{u}: M_{u} \rightarrow B$ is $k$-connected. We choose complementary data $\tilde{f}_{u}, \eta_{u}, P_{u}$ and $\tilde{\nabla}^{u}$ as above. By Proposition 4.8 there exists a geometrisation $\mathcal{G}_{u}$ of the triple $\left(M_{u}, f_{u}, \tilde{\nabla}^{u}\right)$. Given $(M, f)$ with $\operatorname{dim}(M) \leq k-1$ there exists a factorisation up to homotopy

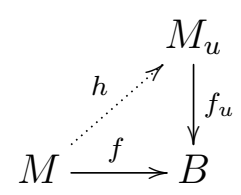

and a refinement of $h$ to a $\operatorname{Spin}^{c}$-map. Then $\mathcal{G}_{M}:=h^{*} \mathcal{G}_{u}$ is a $k$-good geometrisation.

Lemma 4.15 Let $\mathcal{G}_{M}$ be a good geometrisation of $\left(M, f, \tilde{\nabla}^{T M}\right)$. If $(W, F, G)$ is a zero bordism of $(M, f)$ with connection $\tilde{\nabla}^{T W}$, then there exists a geometrisation $\mathcal{G}_{W}$ of $\left(W, F, \tilde{\nabla}^{T W}\right)$ which restricts to $\mathcal{G}_{M}$.

Proof. Since $f_{u}: M_{u} \rightarrow B$ is an $n+1$-equivalence and $\operatorname{dim}(W)=n+1$ we can extend the factorisation $(63)$ to a factorisation

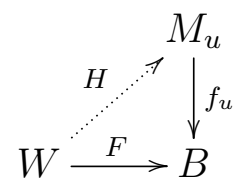

There exists a refinement of $H$ to a stable $\operatorname{Spin}^{c}$-map such that $H \circ i=h$ in the sense $S_{p i n}{ }^{c}$-maps. Then we can define the pull-back $\mathcal{G}_{W}:=H^{*} \mathcal{G}_{u}$ and get $\left(\mathcal{G}_{W}\right)_{\mid M}=\mathcal{G}_{M}$ by the functoriality of the pull-back.

Remark 4.16 Note that Lemma 4.14 does not imply the existence of very good geometrisations. Furthermore observe the following potentially bad behaviour with respect to disjoint unions. Let $\left(M_{i}, f_{i}, \tilde{\nabla}^{T M_{i}}\right), i=0,1$ be two geometric cycles with $k$-good geometrisations $\mathcal{G}_{i}$. Then we can form the disjoint union $\left(M, f, \tilde{\nabla}^{T M}\right):=\left(M_{0}, f_{0}, \tilde{\nabla}^{T M_{0}}\right) \sqcup$ $\left(M_{1}, f_{1}, \tilde{\nabla}^{T M_{1}}\right)$ which carries a geometrisation $\mathcal{G}$ naturally induced by $\mathcal{G}_{i}$. It is not clear that this geometrisation is $k$-good. For this it would good to know that the geomtrizations $\mathcal{G}_{i}$ are pulled back from the same geometrisation $\mathcal{G}_{u}$ by different maps. This is the motivation behind the notion of a very good geometrisation. 


\subsection{An intrinsic formula for $\eta^{a n}$}

The main goal of the present Subsection is to give an intrinsic formula for $\eta^{\text {an }}(x)$ which only involves structures on the cycle $(M, f)$ for $x \in \pi_{n}(M B)_{\text {tors }}$.

The geometric and analytic terms in the formula (30) for $\eta^{a n}(x)$ separately have values in $\mathbb{R} / \mathbb{Z}$; only their sum belongs to $\mathbb{Q} / \mathbb{Z}$. In order to deal with these terms separately it is useful to use a real version $Q_{n}^{\mathbb{R}}(E)$ of the group $Q_{n}(E)$. We start with introducing this group. We further show that there is no loss of information when going over the real version. We let (compare with (5))

$$
U^{\mathbb{R}} \subseteq \operatorname{Hom}^{\text {cont }}\left(K^{0}(E), \pi_{n+1}(K \mathbb{R} / \mathbb{Z})\right)
$$

be the subgroup given by evaluations against elements in $\pi_{n+1}(E \mathbb{R})$ and define

$$
Q_{n}^{\mathbb{R}}(E):=\frac{\operatorname{Hom}^{\text {cont }}\left(K^{0}(E), \pi_{n+1}(K \mathbb{R} / \mathbb{Z})\right)}{U^{\mathbb{R}}} .
$$

The inclusion $\pi_{n+1}(K \mathbb{Q} / \mathbb{Z}) \rightarrow \pi_{n+1}(K \mathbb{R} / \mathbb{Z})$ induces a map

$$
i_{\mathbb{R}}: Q_{n}(E) \rightarrow Q_{n}^{\mathbb{R}}(E)
$$

Lemma 4.17 The map $i_{\mathbb{R}}: Q_{n}(E) \rightarrow Q_{n}^{\mathbb{R}}(E)$ is injective.

Proof. Let $\kappa \in Q_{n}(E)$ be represented by $\hat{\kappa} \in \operatorname{Hom}^{\text {cont }}\left(K^{0}(E), \pi_{n+1}(K \mathbb{Q} / \mathbb{Z})\right)$. Since $\kappa$ is continuous it factors over a finitely generated quotient of $K^{0}(E)$. Hence there exists $N \in \mathbb{N}$ such that $N \hat{\kappa}$ vanishes.

Assume now that $i_{\mathbb{R}}(\kappa)=0$. Then there exists $w \in \pi_{n+1}(E \mathbb{R})$ such that $\hat{\kappa}(\phi)=[\langle w, \phi\rangle] \in$ $\pi_{n+1}(K \mathbb{R} / \mathbb{Z})$ for all $\phi \in K^{0}(E)$. Since $\pi_{n+1}(E \mathbb{R}) \cong \pi_{n+1}(E) \otimes \mathbb{R}$ (see (1)) there exists a finite subset $I \subset \pi_{n+1}(E)$ and a map $\lambda: I \rightarrow \mathbb{R}$ such that $w=\sum_{v \in I} \lambda(v) v$. We have $\hat{\kappa}(\phi)=\sum_{v \in I}[\lambda(v)\langle\phi, v\rangle]$, where here $\langle\phi, v\rangle \in \pi_{n+1}(K)$. For $v \in I$ we define $\hat{v} \in$ $\operatorname{Hom}^{\text {cont }}\left(K^{0}(E), \pi_{n+1}(K)\right)$ by $\hat{v}(\phi):=\langle\phi, v\rangle$. The set $\{\hat{v} \mid v \in I\}$ generates a free abelian subgroup $A \subseteq \operatorname{Hom}^{\text {cont }}\left(K^{0}(E), \pi_{n+1}(K)\right)$. We can choose a minimal subset $J \subseteq I$ which generates a subgroup of $A$ of full rank. Then there exists a suitable map $\mu: J \rightarrow \mathbb{R}$ such that $\hat{\kappa}(\phi)=\sum_{v \in J}[\mu(v) \hat{v}(\phi)]$ for all $\phi \in K^{0}(E)$.

The image of $K^{0}(E) \rightarrow \operatorname{Hom}\left(A, \pi_{n+1}(K)\right)$ has full rank. Hence for every $v \in J$ there exists $\phi_{v} \in K^{0}(E)$ such that $\hat{v}\left(\phi_{v}\right) \neq 0$ and $\hat{v}^{\prime}\left(\phi_{v}\right)=0$ for all $J \ni v^{\prime} \neq v$. It follows that $\hat{\kappa}\left(\phi_{v}\right)=\left[\mu(v) \hat{v}\left(\phi_{v}\right)\right]$. Since $0=N \hat{\kappa}\left(\phi_{v}\right)=\left[N \mu(v) \hat{v}\left(\phi_{v}\right)\right]$ it follows that $\mu(v) \in \mathbb{Q}$. We set $w_{\mathbb{Q}}:=\sum_{v \in J} \mu(v) v \in \pi_{n+1}(E) \otimes \mathbb{Q} \cong \pi_{n+1}(E \mathbb{Q})$. Then we have $\hat{\kappa}(\phi)=\left[\left\langle\phi, w_{\mathbb{Q}}\right\rangle\right]$ for all $\phi \in K^{0}(E)$. This shows that $\hat{\kappa} \in U$ and $\kappa=0$.

Let $x \in \pi_{n}(M B)_{\text {tors }}$ be an $l$-torsion element and $(M, f)$ be a cycle for $x$. We choose a $\operatorname{Spin}^{c}$-extension $\tilde{\nabla}^{T M}$ of the Levi-Civita connection on $M$. We further assume that we have a good geometrisation $\mathcal{G}_{M}$ of $\left(M, f, \tilde{\nabla}^{T M}\right)$ (see Definition 4.12) with a choice of a 
degree-preserving cohomological character $c_{\mathcal{G}}$. If $B$ has finite skeleta, then its existence is guaranteed by Lemma 4.14 .

For every $\phi$ in $K^{0}(B)$ we choose a $\mathbb{Z} / 2 \mathbb{Z}$-graded vector bundle $V_{\phi} \rightarrow M$ such that $\left[V_{\phi}\right]=$ $f^{*} \phi$ in $K^{0}(M)$. We furthermore choose a hermitean metric $h^{V_{\phi}}$ and a metric connection $\nabla^{V_{\phi}}$ so that we get the geometric bundle $\mathbf{V}_{\phi}=\left(V_{\phi}, h^{V_{\phi}}, \nabla^{V_{\phi}}\right)$. It represents a differential $K$-theory class $\left[\mathbf{V}_{\phi}\right] \in \hat{K}^{0}(M)$ such that $I\left(\left[\mathbf{V}_{\phi}\right]\right)=\left[V_{\phi}\right]=f^{*} \phi=I\left(\mathcal{G}_{M}(\phi)\right)$. By the exactness of (49) we get a uniquely determined element

$$
\gamma_{\phi} \in \Omega P^{-1}(M) / \mathrm{im}(\mathbf{c h})
$$

such that

$$
\mathcal{G}_{M}(\phi)-\left[\mathbf{V}_{\phi}\right]=a\left(\gamma_{\phi}\right)
$$

Definition 4.18 We will refer to $\gamma_{\phi}$ as the correction form associated to $\phi$.

Theorem 4.19 The element

$$
i_{\mathbb{R}}\left(\eta^{a n}([M, f])\right) \in Q_{n}^{\mathbb{R}}(M B)
$$

is represented by the homomorphism

$$
K^{0}(M B) \stackrel{\text { Thom }^{K}}{\cong} K^{0}(B) \ni \phi \mapsto\left[-\int_{M} \mathbf{T d}\left(\tilde{\nabla}^{T M}\right) \wedge \gamma_{\phi}\right]-\xi\left(\not D_{M} \otimes \mathbf{V}_{\phi}\right) \in \mathbb{R} / \mathbb{Z}
$$

Proof. The integral in formula $(68)$ belongs to the group $\mathbb{R}\left[b, b^{-1}\right]^{-n-1}$ which will be identified with $\mathbb{R}$ using the generator $b^{-\frac{n+1}{2}}$. First note that, despite of the fact that $\gamma_{\phi}$ is only defined up the image of $\mathbf{c h}: K^{-1}(M) \rightarrow H P \mathbb{Q}^{-1}(M)$, the class

$$
\left[\int_{M} \mathbf{T d}\left(\tilde{\nabla}^{T M}\right) \wedge \gamma_{\phi}\right] \in \mathbb{R} / \mathbb{Z}
$$

is well-defined. Indeed, we have $\langle[M], \mathbf{T d}(T M) \cup \operatorname{ch}(\psi)\rangle \in \mathbb{Z}$ for all $\psi \in K^{-1}(M)$ by the odd version of Atiyah-Singer index theorem. We use (30) in order to express the right-hand side of $(32)$ as

$$
\left[\frac{1}{l} \int_{W} \mathbf{T d}\left(\tilde{\nabla}^{T W}\right) \wedge \mathbf{c h}\left(\nabla^{U_{\phi}}\right)\right]-\xi\left(\not P_{M} \otimes \mathbf{V}_{\phi}\right)
$$

The whole idea is now to turn the integral over $W$ into an integral over $M$. To this end we assume by Lemma 4.15 that the good geometrisation $\mathcal{G}_{M}$ has an extension $\mathcal{G}_{W}$ to $W$. The $K$-theory class $f^{*} \phi$ extends across $W$ as $F^{*} \phi$. We can thus assume, after adding a bundle of the form $\mathbf{W} \oplus \mathbf{W}^{o p}$, that the bundle $\mathbf{V}_{\phi}$ has an extension $\mathbf{U}_{\phi}$ as a geometric bundle to $W$. Note that this sort of stabilisation does not effect the correction form $\gamma_{\phi}$ 
and the reduced $\eta$-invariant $\xi\left(\not D_{M} \otimes \mathbf{V}_{\phi}\right)$. From now on we assume that $\mathbf{V}_{\phi}$ extends. We let $\gamma_{\phi}^{W} \in \Omega P^{-1}(W) / \mathrm{im}(\mathbf{c h})$ be the correction form defined by

$$
\mathcal{G}_{W}(\phi)-\left[\mathbf{U}_{\phi}\right]=a\left(\gamma_{\phi}^{W}\right)
$$

By 62 we conclude that $\left(\gamma_{\phi}^{W}\right)_{\mid \partial W}$ coincides up to the image of $\mathbf{c h}$ with $\gamma_{\phi}$ on all copies of $M$. We now use Stokes' theorem in order to rewrite

$$
\begin{aligned}
{\left[\frac{1}{l} \int_{W} \operatorname{Td}\left(\tilde{\nabla}^{T W}\right) \wedge \mathbf{c h}\left(\nabla^{U_{\phi}}\right)\right] } & =\left[\frac{1}{l} \int_{W} \mathbf{T d}\left(\tilde{\nabla}^{T W}\right) \wedge R\left(\mathcal{G}_{W}(\phi)\right)-\frac{1}{l} \int_{W} \mathbf{T d}\left(\tilde{\nabla}^{T W}\right) \wedge d \gamma_{\phi}^{W}\right] \\
& =\left[\frac{1}{l} \int_{W} \mathbf{T d}\left(\tilde{\nabla}^{T W}\right) \wedge R\left(\mathcal{G}_{W}(\phi)\right)\right]-\left[\int_{M} \mathbf{T d}\left(\tilde{\nabla}^{T M}\right) \wedge \gamma_{\phi}\right]
\end{aligned}
$$

We want to show that homomorphism

$$
\kappa: K^{0}(B) \ni \phi \mapsto\left[\frac{1}{l} \int_{W} \mathbf{T d}\left(\tilde{\nabla}^{T W}\right) \wedge R\left(\mathcal{G}_{W}(\phi)\right)\right] \in \mathbb{R} / \mathbb{Z}
$$

belongs to $U^{\mathbb{R}}$. The integrand of the integral over $W$ can be expressed in terms of the cohomological character $c_{\mathcal{G}_{W}}$ of $\mathcal{G}_{W}$. Therefore $\kappa$ has a factorisation as

$$
K^{0}(B) \stackrel{\mathbf{T d}^{-1} \cup \mathbf{c h}}{\longrightarrow} H P \mathbb{Q}^{0}(B) \stackrel{c_{\mathcal{G}_{W}}}{\longrightarrow} \Omega P_{c l}^{0}(W) \stackrel{\frac{1}{l} \int_{W}}{\longrightarrow} \mathbb{R} \stackrel{[\cdots]_{\mathbb{R} / \mathbb{Z}}}{\longrightarrow} \mathbb{R} / \mathbb{Z}
$$

Since the cohomological character $c_{\mathcal{G}_{W}}$ preserves degree and $\frac{1}{l} \int_{W}$ factorises over the degree $n+1$-part the homomorphism $\kappa$ actually factorizes over

$$
K^{0}(B) \stackrel{p_{n+1}\left(\mathbf{T d}^{-1} \cup \mathbf{c h}\right)}{\longrightarrow} H \mathbb{Q}^{n+1}(B) \stackrel{\left(c_{\mathcal{G}_{W}}\right)_{\mid H \mathbb{Q}^{n+1}(B)}}{\longrightarrow} \Omega_{c l}^{n+1}(W) \stackrel{\frac{1}{l} \int_{W}}{\longrightarrow} \mathbb{R} \stackrel{[\cdots]_{\mathbb{R} / \mathbb{Z}}}{\longrightarrow} \mathbb{R} / \mathbb{Z} .
$$

Since $c_{\mathcal{G}_{W}}$ is continuous, the composition

$$
H \mathbb{Q}^{n+1}(B) \stackrel{\left(c_{\mathcal{G}_{W}}\right)_{\mid H \mathbb{Q}^{n+1}(B)}}{\longrightarrow} \Omega_{c l}^{n+1}(W) \stackrel{\frac{1}{l} \int_{W}}{\longrightarrow} \mathbb{R}
$$

is continuous and therefore given by the pairing against an element of $H \mathbb{R}_{n+1}(B)$. We now use the $\mathbb{R}$-version of $(13)$ in order to conclude that $\kappa \in U^{\mathbb{R}}$. Therefore $i_{\mathbb{R}}\left(\eta^{\text {an }}([M, f])\right)$ is also represented by the map (68).

Remark 4.20 Let us mention the following aspect of the intrinsic formula (68) which is not yet completely understood at the moment. For the intrinsic formula to make sense we do not need the zero bordism $(W, F)$ of $l$-copies of $(M, f)$. Therefore formula $(68)$ provides an element $\eta_{\mathcal{G}}^{\text {intrinsic }}\left(M, f, \tilde{\nabla}^{T M}\right) \in Q_{n}^{\mathbb{R}}(M B)$. One can check that $\eta_{\mathcal{G}}^{\text {intrinsic }}\left(M, f, \nabla^{T M}\right)=0$ if the data $\left(M, f, \tilde{\nabla}^{T M}, \mathcal{G}_{M}\right)$ extends to a zero bordism. Note that in general two good geometrisations of $\left(M, f, \tilde{\nabla}^{T M}\right)$ cannot be connected over the cylinder but this is possible if both are very good with respect to the same universal geometrisation. More general, if we work with very good geometrisation associated to a fixed universal geometrisation 
(see Remark 4.13), then the problem with disjoint unions mentioned in Remark 4.16 disappears, too. Therefore, if we fix a universal geometrization, then we would get a homomorphism

$$
\eta_{\mathcal{G}}^{\text {intrinsic }}: \pi_{n}(M B) \rightarrow Q_{n}^{\mathbb{R}}(M B)
$$

which restricts to $i_{\mathbb{R}} \circ \eta^{\text {top }}=i_{\mathbb{R}} \circ \eta^{a n}$ on $\pi_{n}(M B)_{t o r s}$. In general we do not know the topological contents of this extension of the universal $\eta$-invariant. It might be related to the effect observed in Remark 5.21.

\section{$5 \quad$ Examples}

\subsection{Adams' $e$-invariant}

We consider the example $B=*$. The associated Thom spectrum is the sphere spectrum $S \cong M B$. By Serre's theorem [51] the homotopy groups $\pi_{n}(S)$ are finite for $n \geq 1$ (we refer to [48] for more details about their structure). Therefore the universal $\eta$-invariant is defined on all of $\pi_{n}(S)$.

We have an identification $K^{0}(S) \cong \mathbb{Z}$. Furthermore, since $\pi_{n+1}(S \mathbb{Q}) \cong 0$ for $n \geq 0$ the group $U$ defined in (5) is trivial. From now one let $n \in \mathbb{N}$ be odd. After identifying $\pi_{n+1}(K \mathbb{Q} / \mathbb{Z}) \cong \mathbb{Q} / \mathbb{Z}$ we obtain the identification

$$
Q_{n}(S) \cong \operatorname{Hom}(\mathbb{Z}, \mathbb{Q} / \mathbb{Z}) \cong \mathbb{Q} / \mathbb{Z}
$$

given by evaluation against $1 \in \mathbb{Z}$. For every odd $n \in \mathbb{N}$ the universal $\eta$-invariant is thus interpreted as a homomorphism

$$
\eta: \pi_{n}(S) \rightarrow \mathbb{Q} / \mathbb{Z}
$$

The universal $\eta$-invariant essentially coincides with Adams' e-invariant [1]

$$
e^{\text {Adams }}: \pi_{n}(S) \rightarrow \mathbb{Q} / \mathbb{Z}
$$

which was introduced in order to detect the image of the $J$-homomorphism

$$
J: K O_{n+1} \rightarrow \pi_{n}^{S} .
$$

We will see below that we have the relation

$$
\eta=e_{\mathbb{C}}^{\text {Adams }}:=\left\{\begin{array}{cc}
e^{\text {Adams }} & \frac{n-1}{2} \text { even } \\
2 e^{\text {Adams }} & \frac{n-1}{2} \text { odd }
\end{array} .\right.
$$

We consider the Adams filtration of $\pi_{*}(S)$ associated to the $K$-theory based Adams spectral sequence (see e.g. [3] or Section 2.4). It follows from Proposition 2.7, 4., that the universal $\eta$-invariant (69) induces an injection

$$
\eta^{\text {top }}: \operatorname{Gr}^{1} \pi_{n}(S) \hookrightarrow \mathbb{Q} / \mathbb{Z}
$$


(recall that $n$ is assumed to be odd). The relation of the Adams e-invariant to spectral geometry has first been observed in [7]. The spectral geometric calculation of the Adams $e$-invariant interprets $\pi_{n}(S)$ as a framed bordism group. It has the favourable property that it provides an intrinsic formula for $e^{\text {Adams }}$, a fact which has been successfully exploited e.g. in [26], [50].

The goal of the following discussion is to derive, using Theorems 4.19 and 3.6, the intrinsic formula $(73)$ for $\eta^{\text {top }}$ and compare it with the formula [6, Thm 4.14] for $e_{\mathbb{C}}^{\text {Adams }}$. This is finally our argument for (71).

We let $(M, f)$ be a cycle for $\pi_{n}(S)$ as in Subsection 3.2, where the constant map $f: M \rightarrow *$ is refined to a stable framing $T M \oplus\left(M \times \mathbb{R}^{k}\right) \cong M \times \mathbb{R}^{n+k}$ of the tangent bundle. A tangential $\operatorname{Spin}^{c}$-structure is now given by a trivialisation

$$
P(T M) \otimes Q(k) \cong M \times \operatorname{Spin}^{c}(n+k)
$$

We will in fact assume that $P(T M)$ comes from a Spin-structure. In this case the LeviCivita connection induces a canonical $\operatorname{Spin}^{c}$-connection $\tilde{\nabla}^{T M}$.

In order to apply Theorem 4.19 we must first choose a good geometrisation of $\left(M, f, \tilde{\nabla}^{T M}\right)$. We will use the notation of Subsection 4.4. We can choose the manifold $M_{u}$ to be a point. The datum $\left(M_{u}, f_{u}, \tilde{\nabla}\right)$ (where $f_{u}: M_{u} \rightarrow *$ is constant and $\tilde{\nabla}$ is the trivial connection on the trivial bundle $P_{u}$ ) has a unique geometrisation $\mathcal{G}_{u}$. Let $h: M \rightarrow M_{u}$ be the constant map. The $\operatorname{Spin}^{c}$-bundle $h^{*} P_{u}$ is trivial. Hence the given trivialisation (72) refines $h$ to a $\operatorname{Spin}^{c}$-map. Using this refinement we define the geometrisation $\mathcal{G}:=h^{*} \mathcal{G}_{u}$ which turns out to be $\ell$-good for every $\ell \in \mathbb{N}$.

For $1 \in K^{0}(*) \cong \mathbb{Z}$ we have $\mathcal{G}_{u}(1)=1 \in \hat{K}^{0}\left(M_{u}\right)$. We now use Lemma 4.9 in order to calculate $\mathcal{G}(1) \in \hat{K}^{0}(M)$. By equation 60 we have

$$
\mathcal{G}(1)=1+a\left(\frac{\tilde{\mathbf{T}} \mathbf{d}\left(\tilde{\nabla}^{t r i v}, \tilde{\nabla}^{T M}\right)}{\mathbf{T d}\left(\tilde{\nabla}^{T M}\right)}\right),
$$

where $\nabla^{\text {triv }}$ is the connection on $P(T M) \otimes Q(k)$ induced by the trivialization $(72)$. Let $\mathbf{V}_{1}$ be the trivial one-dimensional geometric bundle on $M$. Then $\left[\mathbf{V}_{1}\right]=1 \in \hat{K}^{0}(M)$ and in view of Equation (67) we must take the correction form

$$
\gamma_{1}:=\frac{\tilde{\mathbf{T}} \mathbf{d}\left(\tilde{\nabla}^{t r i v}, \tilde{\nabla}^{T M}\right)}{\boldsymbol{T d}\left(\tilde{\nabla}^{T M}\right)} .
$$

We now specialize Theorem 4.19 to the present situation and obtain the intrinsic formula

$$
i_{\mathbb{R}}\left(\eta^{a n}([M, f])\right)(1)=\left[-\int_{M} \tilde{\mathbf{T}} \mathbf{d}\left(\tilde{\nabla}^{t r i v}, \tilde{\nabla}^{T M}\right)\right]-\xi\left(\not D_{M}\right) \in \mathbb{R} / \mathbb{Z}
$$

This formula directly compares with the formula for $e_{\mathbb{C}}^{\text {Adams }}([M, f])$ derived by specializing [6. Thm 4.14]. 


\section{$5.2 \rho$-invariants and the index theorem for flat bundles}

We consider a closed odd-dimensional $\operatorname{Spin}^{c}$-manifold $M$. The geometry on $M$ is given by a Riemannian metric and the Spin $^{c}$-extension of the Levi-Civita connection. If $\mathbf{V}=$ $\left(V, \nabla^{V}, h^{V}\right)$ is a flat hermitean vector bundle of dimension $k$ on $M$, then the difference of reduced $\eta$-invariants

$$
\rho\left(\not D_{M}, \mathbf{V}\right):=\xi\left(\not D_{M} \otimes \mathbf{V}\right)-k \xi\left(\not D_{M}\right)
$$

is invariant under variations of the geometry of $M$. The $\rho$-invariant is thus a differential topological invariant of the $\operatorname{Spin}^{c}$-manifold $M$ with a flat hermitean bundle $\mathbf{V}$.

The $\rho$-invariant is a classical example of a topological invariant derived from the $\eta$ invariant which has been studied a lot. For example, it has been used successfully to detect elements in Spin $^{c}$-bordism groups of classifying spaces of finite cyclic groups [11], [10]. We refer to these reference for examples of explicit calculations of $\rho$-invariants.

The precise homotopy theoretic description of $\rho$-invariants is given by the index theorem for flat bundles [7, Thm. 5.3]. The goal of the following discussion is to explain the relation of the relation $\eta^{a n}=\eta^{\text {top }}$ shown in Theorem 3.6 with the index theorem for flat bundles. Roughly speaking, this goes as follows. The index theorem for flat bundles is about the pairing of the $K$-homology class represented by the Spin $^{c}$-Dirac operator with the torsion $K$-cohomology classes obtained from the flat bundle, while our index theorem considers the pairing of a torsion $K$-homology class with $K$-theory classes. Clearly the case of intersection is when both classes are torsion.

We first translate the data of the $\operatorname{Spin}^{c}$-manifold $M$ of odd dimension $n$ with a flat hermitean bundle $\mathbf{V}$ into the bordism picture. Let $U(k)^{\delta}$ denote the unitary group equipped with the discrete topology. Its classifying space $B U(k)^{\delta}$ is universal for flat hermitean vector bundles of dimension $k$. We consider the bordism group based on the Thom spectrum of

$$
B:=\operatorname{SPpin}^{c} \times B U(k)^{\delta} \stackrel{\mathrm{pr}}{\rightarrow} B \operatorname{Spin}^{c} .
$$

We let $(M, f)$ be a cycle for $\pi_{n}\left(M S p i n^{c}\right)$ and consider in addition a map $g: M \rightarrow B U(k)^{\delta}$ which classifies a flat bundle $\mathbf{V}$. In this way we get a class

$$
[M,(f, g)] \in \pi_{n}(M B)
$$

We assume that this class is torsion in order to apply the universal $\eta$-invariant.

We consider the $K$-theory class $\lambda_{k} \in K^{0}\left(B U(k)^{\delta}\right)$ of the universal $\mathbb{C}^{k}$-bundle on $B U(k)^{\delta}$ and the projection

$$
q: B \operatorname{Spin}^{c} \times B U(k)^{\delta} \rightarrow B U(k)^{\delta} .
$$

Since $\operatorname{ch}\left(\lambda_{k}-k\right)=0$ the evaluation against the difference $q^{*} \lambda_{k}-k$ provides a well-defined homomorphism

$$
\mathrm{ev}_{q^{*} \lambda_{k}-k}: Q_{n}(M B) \rightarrow \mathbb{Q} / \mathbb{Z}
$$

Lemma 5.1 We assume that $[M,(f, g)] \in \pi_{n}(M B)$ is a torsion class. Then we have the equality

$$
\mathrm{ev}_{q^{*} \lambda_{k}-k}\left(\eta^{a n}([M,(f, g)])\right)=\rho\left(\not D_{M}, \mathbf{V}\right)
$$


Proof. We are going to use the notation introduced in Subsection 3.4. As an intermediate step we choose, for a suitable non-vanishing integer $l$, a zero bordism $(W,(F, G))$ of the union of $l$ copies of the cycle $(M,(f, g))$ with $\operatorname{Spin}^{c}$-geometry. The geometric bundle $\mathbf{U}$ is then the flat hermitean bundle classified by $F$, and we have by Definition 3.5

$$
\operatorname{ev}_{q^{*} \lambda_{k}-k}\left(\eta^{a n}([M,(f, g)])\right)=\left[\frac{1}{l} \operatorname{index}\left(\not D_{W} \otimes \mathbf{U}\right)\right]-\left[\frac{k}{l} \operatorname{index}\left(\not D_{W}\right)\right] .
$$

If we use (30) instead, then we express this evaluation in terms of an integral over local data on $W$ and the reduced $\eta$-invariants. Because of $\operatorname{ch}\left(\nabla^{U}\right)=k$ the local contributions cancel out and we remain with

$$
\mathrm{ev}_{q^{*} \lambda_{k}-k}\left(\eta^{a n}([M,(f, g)])\right)=\rho\left(\not D_{M}, \mathbf{V}\right)
$$

We now calculate the topological version of the universal $\eta$-invariant explicitly. We again assume that $x=[M,(f, g)] \in \pi_{n}(M B)$ is a torsion element and let $\tilde{x} \in \pi_{n+1}(K \mathbb{Q} / \mathbb{Z} \wedge M B)$ as in (3). By definition of $\eta^{\text {top }}$ we get the equality

$$
\operatorname{ev}_{q^{*} \lambda_{k}-k}\left(\eta^{t o p}([M,(f, g)])\right)=\left\langle\operatorname{Thom}^{K}\left(q^{*} \lambda_{k}-k\right), \tilde{x}\right\rangle \text {. }
$$

The right-hand side of $(76)$ is the analytic side of the index theorem for flat bundles in [7, Thm. 5.3]. The topological side of the index theorem for flat bundles [7, Thm. 5.3] is not given as the pairing of a $K \mathbb{Q} / \mathbb{Z}$-homology class with a $K$-theory class, but rather by a pairing between a $K$-homology class and a $K \mathbb{R} / \mathbb{Z}$-cohomology class. In the following we rewrite the right-hand side of $(77)$ in this way.

There exists a class $\Lambda_{k} \in K \mathbb{R} / \mathbb{Z}^{-1}\left(B U(k)^{\delta}\right)$ with $\partial \Lambda_{k}=\lambda_{k}-k$, where $\partial$ is the Bockstein operator $\partial: K \mathbb{R} / \mathbb{Z}^{-1}\left(B U(k)^{\delta}\right) \rightarrow K^{0}\left(B U(k)^{\delta}\right)$, and which is uniquely characterised by the following property: If $h: N \rightarrow B U(k)^{\delta}$ is a map from a smooth manifold $N$, then $h^{*}\left(\Lambda_{k}\right)=[\mathbf{U}]-k$, where $\mathbf{U}$ is the unitary flat bundle classified by $h$. In order to interpret this equality we employ the identification $K \mathbb{R} / \mathbb{Z}^{-1}(N) \cong \hat{K}_{\text {flat }}^{0}(N)$ given by 48 .

Recall that $q$ denotes the projection (75). We use Lemma 2.6 for the first equality in the chain

$$
\mathrm{ev}_{q^{*} \lambda_{k}-k}\left(\eta^{t o p}(x)\right)=\left\langle\operatorname{Thom}^{K \mathbb{R} / \mathbb{Z}}\left(q^{*} \Lambda_{k}\right), \epsilon(x)\right\rangle=\left\langle q^{*} \Lambda_{k}, \operatorname{Thom}_{K}(\epsilon(x))\right\rangle=\left\langle[\mathbf{V}]-k,[M]_{K}\right\rangle,
$$

where $[M]_{K}$ denotes the $K$-theory fundamental class of the $\operatorname{Spin}^{c}$-manifold $M$. The righthand side of this equality is the topological side of the index theorem for flat bundles of [7, Thm. 5.3]. The following Corollary now immediately follows from the equality $\eta^{t o p}=\eta^{a n}$.

Corollary 5.2 Let $n \in \mathbb{N}$ be odd and $M$ be a closed $n$-dimensional Spin ${ }^{c}$-manifold with a flat hermitean $k$-dimensional vector bundle $\mathbf{V}$. We assume in addition that the corresponding class $[M,(f, g)] \in \pi_{n}\left(M\left(B \operatorname{Spin}^{c} \times B U(k)^{\delta}\right)\right.$ is torsion. Then we have the following equality in $\mathbb{R} / \mathbb{Z}$ :

$$
\rho\left(\not D_{M}, \mathbf{V}\right)=\left\langle[\mathbf{V}]-k,[M]_{K}\right\rangle
$$


In this way Theorem 4.19 implies a special case of [7, Thm. 5.3]. Let us again remark, that by [7, Thm. 5.3] the equality (78) holds true without the additional assumption that $[M,(f, g)]$ is a torsion class.

\subsection{Algebraic $K$-theory}

In this Subsection we use the universal $\eta$-invariant in order detect algebraic $K$-theory classes of $\mathbb{C}$. We will observe that for odd $n \in \mathbb{N}$ the well-known homomorphism

$$
\varepsilon: K_{n}(\mathbb{C}) \rightarrow \mathbb{Q} / \mathbb{Z}
$$

(see (83) ) can be obtained from an appropriate evaluation of the universal $\eta$-invariant. Our main result is Theorem 5.5 which provides a formula for $\varepsilon$ in terms of geometric cycles for $K$-theory classes. We will explain how the results of [47] and [39] can be interpreted as constructions with the universal $\eta$-invariant.

For $n \in \mathbb{N}$ the algebraic $K$-theory groups $K_{n}(\mathbb{C})$ of the field $\mathbb{C}$ are defined as the homotopy groups of the connective algebraic $K$-theory spectrum $K(\mathbb{C})$. This spectrum is connected with classifying spaces through Quillen's +-construction (see [2, Ch. 3] for a detailed description)

$$
p: B G L\left(\mathbb{C}^{\delta}\right) \rightarrow B G L\left(\mathbb{C}^{\delta}\right)^{+}
$$

by the equivalence

$$
\Omega^{\infty} K(\mathbb{C}) \cong \mathbb{Z} \times B G L\left(\mathbb{C}^{\delta}\right)^{+}
$$

There exists a class

$$
\Lambda_{0} \in K \mathbb{R} / \mathbb{Z}^{-1}\left(B G L\left(\mathbb{C}^{\delta}\right)^{+}\right)
$$

which is uniquely characterised by the following property: If $k \in \mathbb{N}$ and $g_{k}: N \rightarrow$ $B G L\left(k, \mathbb{C}^{\delta}\right)$ is a map from a smooth manifold, then

$$
\iota_{*}\left(p \circ i_{k} \circ g_{k}\right)^{*} \Lambda_{0}=[\mathbf{V}]-k-a\left(\operatorname{Re}\left(\tilde{\mathbf{c h}}\left(\nabla^{V, u}, \nabla^{V}\right)\right)\right) \in K \mathbb{C} / \mathbb{Z}^{-1}(N),
$$

where on the right-hand side we use the identification of $K \mathbb{C} / \mathbb{Z}^{-1}(N)$ with the flat part of the complex version of differential $K$-theory mentioned in Remark $4.1, i_{k}: B G L\left(k, \mathbb{C}^{\delta}\right) \rightarrow$ $B G L\left(\mathbb{C}^{\delta}\right)$ is the canonical map, $\mathbf{V}=\left(V, \nabla^{V}\right)$ is the flat complex vector bundle of dimension $k$ classified by $g_{k}, \nabla^{V, u}$ is some choice of unitarisable connection on $V$, and $\iota: K \mathbb{R} / \mathbb{Z}^{-1} \rightarrow K \mathbb{C} / \mathbb{Z}^{-1}$ is the canonical map. We further define

$$
\Theta_{0}:=\partial \Lambda_{0} \in K^{0}\left(B G L\left(\mathbb{C}^{\delta}\right)^{+}\right),
$$

where $\partial: K \mathbb{R} / \mathbb{Z}^{-1} \rightarrow K^{0}$ is the Bockstein operator.

We will consider the Thom spectrum $M B$ associated to the projection

$$
B:=B \operatorname{Spin}^{c} \times B G L\left(\mathbb{C}^{\delta}\right)^{+} \rightarrow B \operatorname{Spin}^{c} .
$$

Furthermore we let $q: B \rightarrow B G L\left(\mathbb{C}^{\delta}\right)^{+}$be the projection. Let $n \in \mathbb{N}$ be odd. Since $\mathbf{c h}\left(\Theta_{0}\right)=0$, by Lemma 2.5 the second map in the following definition of $\varepsilon_{0}$ is well-defined:

$$
\varepsilon_{0}: \pi_{n}(M B)_{\text {tors }} \stackrel{\eta^{\text {top }}}{\rightarrow} Q_{n}(M B) \stackrel{\text { ev }_{q^{*} \Theta_{0}}}{\rightarrow} \mathbb{Q} / \mathbb{Z}
$$


We consider a cycle $(M, f)$ for $\pi_{n}\left(M S \sin ^{c}\right)$ and a flat complex vector bundle $\mathbf{V}$ on $M$ of dimension $k$. It is classified by a map $g_{k}: M \rightarrow B G L\left(k, \mathbb{C}^{\delta}\right)$, and we consider the induced map

$$
g_{0}:=p \circ i_{k} \circ g_{k}: M \rightarrow B G L\left(\mathbb{C}^{\delta}\right)^{+} .
$$

Then $\left(M,\left(f, g_{0}\right)\right)$ is a cycle for $\pi_{n}(M B)$.

Theorem 5.3 We assume that $\left[M,\left(f, g_{0}\right)\right] \in \pi_{n}(M B)_{\text {tors. }}$. If $\mathbf{V}$ carries a flat hermitean metric, then

$$
\varepsilon_{0}\left(\left[M,\left(f, g_{0}\right)\right]\right)=\rho\left(\not D_{M}, \mathbf{V}\right)
$$

In general we have

$$
\varepsilon_{0}\left(\left[M,\left(f, g_{0}\right)\right]\right)=\left\langle[M]_{K}, g_{0}^{*} \Lambda_{0}\right\rangle
$$

Proof. We first prove the general case 82 by the following chain of equalities

$$
\begin{array}{rll}
\varepsilon_{0}\left(\left[M,\left(f, g_{0}\right)\right]\right) & \stackrel{\text { Def }}{=} & \operatorname{ev}_{q^{*} \Theta_{0}}\left(\eta^{t o p}\left(\left[M,\left(f, g_{0}\right)\right]\right)\right) \\
\text { Lemma } \stackrel{2.6}{=} & \left\langle\operatorname{Thom}^{K \mathbb{R} / \mathbb{Z}}\left(q^{*} \Lambda_{0}\right), \epsilon\left(\left[M, f, g_{0}\right]\right)\right\rangle \\
= & \left\langle g_{0}^{*} \Lambda_{0},[M]_{K}\right\rangle .
\end{array}
$$

In the unitary case we observe that $g_{0}^{*} \Lambda_{0}=[\mathbf{V}]-k$. The equality 81 now follows from (78) and the chain of equalities

$$
\varepsilon_{0}\left(\left[M,\left(f, g_{0}\right)\right]\right) \stackrel{[82}{=}\left\langle g_{0}^{*} \Lambda_{0},[M]_{K}\right\rangle=\left\langle[\mathbf{V}]-k,[M]_{K}\right\rangle \stackrel{[88}{=} \rho\left(\not D_{M}, \mathbf{V}\right)
$$

Note that in the present paper we have shown (78) under the assumption that $\left[M,\left(f, g_{k}^{u}\right)\right]$ is a torsion class in $\pi_{n}\left(M\left(B \operatorname{Spin}_{+}^{c} \times B U(k)^{\delta}\right)\right)$, where $g_{k}^{u}: M \rightarrow B U(k)^{\delta}$ classifies the hermitean flat bundle $\mathbf{V}$. Since this might not be the case in general we have to appeal to the proof of this formula (78) without such an assumption given in [7, Thm. 5.3].

We have a canonical map of spectra

$$
\Theta: K(\mathbb{C}) \rightarrow K
$$

Assume again that $n \in \mathbb{N}$ is odd. Since $\mathbf{c h} \circ \Theta=\operatorname{dim}$ is concentrated in degree zero, by Lemma 2.5 the evaluation in the second map of the following definition is well-defined

$$
\varepsilon: K_{n}(\mathbb{C})_{\text {tors }} \stackrel{\eta^{\text {top }}}{\rightarrow} Q_{n}(K(\mathbb{C})) \stackrel{\mathrm{ev}_{q^{*} \Theta}}{\rightarrow} \mathbb{Q} / \mathbb{Z}
$$

We know from Suslin [52, Thm 4.9] that the map $\varepsilon$ induces an isomorphism

$$
K_{n}(\mathbb{C})_{\text {tors }} \cong\left\{\begin{array}{cc}
\mathbb{Q} / \mathbb{Z} & n \text { odd } \\
0 & n \text { even }
\end{array}\right.
$$

in the case that $n$ is odd, and that the kernel of $\varepsilon$ is a uniquely divisible group. 
Our goal is to provide a formula for $\varepsilon$ using geometric cycles for algebraic $K$-theory classes of $\mathbb{C}$. Let $(M, f)$ be a cycle for $\pi_{n}(S)$, where $f$ stands for the constant map $M \rightarrow *$ refined by a stable normal framing. Furthermore, let $\mathbf{V}$ be a flat complex vector bundle of dimension $k$ classified by a map $g_{k}: M \rightarrow B G L\left(k, \mathbb{C}^{\delta}\right)$. Using the equivalence (80) we define

$$
g:=(\{0\} \times \mathrm{id}) \circ p \circ i_{k} \circ g_{k}: M \rightarrow \Omega^{\infty} K(\mathbb{C}) .
$$

In this way we get a class $[M,(f, g)] \in \pi_{n}\left(\Sigma_{+}^{\infty} \Omega^{\infty} K(\mathbb{C})\right)$. Employing the canonical map $u: \Sigma_{+}^{\infty} \Omega^{\infty} K(\mathbb{C}) \rightarrow K(\mathbb{C})$ we can form the class

$$
u_{*}[M,(f, g)] \in K_{n}(\mathbb{C}) .
$$

Remark 5.4 Every element $x \in K_{n}(\mathbb{C})_{\text {tors }}$ can be represented geometrically in this way. More precisely there exists $(M,(f, g))$ as above such that $x=u_{*}[M,(f, g)]$ and $[M,(f, g)] \in \pi_{n}\left(\Sigma_{+}^{\infty} \Omega^{\infty} K(\mathbb{C})\right)_{\text {tors }}$. In order to see this first represent $x$ by a map $\gamma$ : $S^{n} \rightarrow \Omega^{\infty} K(\mathbb{C})$. Using the standard normal framing of $S^{n}$ this map represents a torsion class $\left[S^{n},(f, \gamma)\right] \in \pi_{n}\left(\Sigma_{+}^{\infty} \Omega^{\infty} K(\mathbb{C})\right)_{\text {tors }}$. Since $\Sigma_{+}^{\infty}(p)$ is an equivalence by the universal property of the +-construction there exists a class $z \in \pi_{n}\left(\Sigma_{+}^{\infty} B G L\left(\mathbb{C}^{\delta}\right)\right)_{\text {tors }}$ such that $p_{*}(z)=\left[S^{n},(f, \gamma)\right]$. Finally, the class $z$ can be represented in the form $z=\left[M,\left(f, i_{k} \circ g_{k}\right)\right]$ for some $k \in \mathbb{N}$. Using this data we get $u_{*}[M,(f, g)]=x$.

Theorem 5.5 We assme that $[M,(f, g)] \in \pi_{n}\left(\Sigma_{+}^{\infty} \Omega^{\infty} K(\mathbb{C})\right)_{\text {tors }}$. If $\mathbf{V}$ carries a flat hermitean metric, then

$$
\varepsilon\left(u_{*}[M,(f, g)]\right)=\rho\left(D_{M}, \mathbf{V}\right)+k e_{\mathbb{C}}^{\text {Adams }}([M, f]) .
$$

In general we have

$$
\varepsilon\left(u_{*}[M,(f, g)]\right)=\left\langle g_{0}^{*} \Lambda_{0},[M]_{K}\right\rangle+k e_{\mathbb{C}}^{\text {Adams }}([M, f]) .
$$

Proof. We again start with the general case (86). We calculate

$$
\begin{aligned}
\varepsilon\left(u_{*}[M,(f, g)]\right) & =\operatorname{ev}_{\Theta}\left(\eta^{t o p}\left(u_{*}[M,(f, g)]\right)\right) \\
& =\operatorname{ev}_{u^{*} \Theta}\left(\eta^{t o p}([M,(f, g)])\right) \\
& =\operatorname{ev}_{i_{k}^{*} u^{*} \Theta}\left(\eta^{t o p}\left(\left[M,\left(f, g_{0}\right)\right]\right)\right) \\
& =\operatorname{ev}_{\Theta_{0}+k}\left(\eta^{t o p}\left(\left[M,\left(f, g_{0}\right)\right]\right)\right) .
\end{aligned}
$$

We can now use the unstable case 82 and 71 in order to deduce

$$
\varepsilon\left(u_{*}([M,(f, g)])\right)=\left\langle g_{0}^{*} \Lambda_{0},[M]_{K}\right\rangle+k e_{\mathbb{C}}^{\text {Adams }}([M, f]) .
$$

The unitary case 850 now follows from (86) and 81). 
Remark 5.6 We now show how one can deduce a special case of [39, Thm A] from (86). In [39] an algebraic $K$-theory class is constructed from a homology sphere $M$ of dimension $n$ and a representation $\alpha: \pi_{1}(M) \rightarrow G L\left(k, \mathbb{C}^{\delta}\right)$. One gets an induced map

$$
\tilde{g}: M \stackrel{\alpha}{\rightarrow} B G L\left(k, \mathbb{C}^{\delta}\right) \stackrel{i_{k}}{\rightarrow} B G L\left(\mathbb{C}^{\delta}\right)
$$

to which Quillen's +-construction is applied. The fundamental group of a homology sphere is perfect so that the +-construction $M^{+}$of $M$ is homotopy equivalent to a simplyconnected homology sphere, hence to $S^{n}$. Thus we get a map $g^{+}: S^{n} \simeq M^{+} \stackrel{\tilde{g}^{+}}{\rightarrow} B G L\left(\mathbb{C}^{\delta}\right)^{+}$ which represents a class $\left[g^{+}\right] \in K_{n}(\mathbb{C})$. The homology sphere $M$ admits a stable normal framing (see e.g. [41] or [28, Lem. 1]) which refines the constant map $f: M \rightarrow *$. We further use the composition $g: M \rightarrow M^{+} \stackrel{\tilde{g}^{+}}{\rightarrow} B G L\left(\mathbb{C}^{\delta}\right)^{+}$in order to define the class $[M,(f, g)] \in \pi_{n}\left(\Sigma_{+}^{\infty} \Omega^{\infty} K(\mathbb{C})_{+}\right)$such that $u_{*}[M,(f, g)]=\left[g^{+}\right]$.

We consider the map of fibre sequence

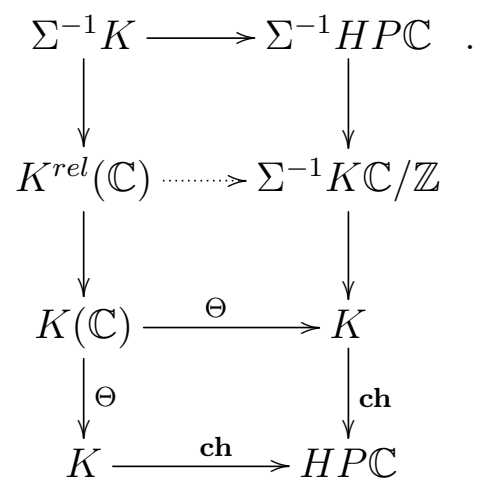

(the left column defines the relative $K$-theory spectrum $K^{\text {rel }}(\mathbb{C})$ ). For odd $n \in \mathbb{N}$ the dotted horizontal arrow induces a map

$$
e: K_{n}(\mathbb{C}) \cong \frac{\pi_{n}\left(K^{\text {rel }}(\mathbb{C})\right)}{\operatorname{im}\left(\pi_{n}\left(\Sigma^{-1} K\right) \rightarrow \pi_{n}\left(K^{\text {rel }}(\mathbb{C})\right)\right)} \rightarrow \pi_{n}\left(\Sigma^{-1} K \mathbb{C} / \mathbb{Z}\right) \cong \mathbb{C} / \mathbb{Z}
$$

Let $\mathbf{V}^{\text {flat }}$ denote the flat vector bundle determined by the representation $\alpha$. The statement of [39, Thm A] is the equality

$$
e\left(\left[g^{+}\right]\right)=\rho_{\mathbb{C}}\left(\not D_{M}, \mathbf{V}^{\text {flat }}\right)
$$

in $\mathbb{C} / \mathbb{Z}$. The subscript $\mathbb{C}$ on the right-hand side indicates a complex version of the $\rho$ invariant defined for flat vector bundles without requiring a flat hermitean metric. We have the equality

$$
\operatorname{Re}\left(\rho_{\mathbb{C}}\left(\not D_{M}, \mathbf{V}^{f l a t}\right)\right)=\left\langle g^{*} \Lambda_{0},[M]_{K}\right\rangle .
$$

Note that $(\mathbb{C} / \mathbb{Z})_{\text {tors }}=\mathbb{Q} / \mathbb{Z} \subset \mathbb{R} / \mathbb{Z} \subset \mathbb{C} / \mathbb{Z}$. If $\left[g^{+}\right] \in K_{n}(\mathbb{C})$ is a torsion class, then using Lemma 2.6 we get the identification

$$
e\left(\left[g^{+}\right]\right)=\varepsilon\left(\left[g^{+}\right]\right)-k e_{\mathbb{C}}^{\text {Adams }}([M, f]) \in \mathbb{R} / \mathbb{Z}
$$

by an argument which is similar to the calculation (87). Hence if $\left[g^{+}\right]$is a torsion class, then the real part of (89) is equivalent to 86 . 
Remark 5.7 Let us comment on the fact that Adams' $e$-invariant appears on the righthand sides of $(85)$ and $(86)$. Note that $K(\mathbb{R})$ is a ring spectrum with unit $\epsilon_{K(\mathbb{R})}: S \rightarrow$ $K(\mathbb{R})$. The unit induces a homomorphism $\pi_{*}(S) \rightarrow K_{n}(\mathbb{R})$. Since the image $\operatorname{im}(J)$ of the $J$-homomorphism (70) is a well-known summand of $\pi_{*}(S)$ it was an interesting question to determine its image under the unit $\epsilon_{K(\mathbb{R})}$. Let us consider the case

$$
\frac{\mathbb{Z}}{\frac{B_{m}}{4 m} \mathbb{Z}} \cong \operatorname{im}(J)_{4 m-1} \subseteq \pi_{4 m-1}(S)
$$

for $m \in \mathbb{N}$. In [47] it has been shown that this piece goes injectively to algebraic $K$-theory. This was deduced from the following two facts:

1. $\operatorname{im}(J)_{4 m-1}$ is detected by (the real version of) Adams' $e$-invariant $e: \pi_{4 m-1}(S) \rightarrow$ $\mathbb{Q} / \mathbb{Z}$.

2. The $e$-invariant has a factorisation over the analog

$$
K_{n}(\mathbb{R})(\ldots) \rightarrow \pi_{n}\left(\Sigma^{-1} K O \mathbb{C} / \mathbb{Z}\right)
$$

of the homomorphism 88 .

The complex case of this factorisation is easily seen from Theorem 5.5. In fact the elements in $\operatorname{im}(J)_{4 m-1}$ can be represented by cycles of the form $u_{*}\left[S^{4 m-1},(f, g)\right]$, where $f$ carries a normal framing obtained from the standard framing by twisting with an element of $\pi_{4 m-1}(O)$, and $g$ is obtained from the classifying map of the trivial one-dimensional bundle V. Then we get from Theorem 5.5, (85),

$$
\varepsilon\left(u_{*}\left[S^{4 m-1},(f, g)\right]\right)=e_{\mathbb{C}}^{\text {Adams }}\left(\left[S^{4 m-1}, f\right]\right) .
$$

\subsection{String-bordism}

In this Subsection we describe the connection of the present paper with constructions in [21. In this reference we introduced an invariant $b^{a n}$ of elements of the bordism group $\pi_{4 m-1}($ MString) using a formula which shares a lot of similarities with the intrinsic formula 68 for $\eta^{a n}$. We will recall the definition of the complex version $b_{\mathbb{C}}^{a n}$ during the proof of Theorem 5.9 .

The spectrum of topological modular forms tm $f$ has been constructed by Miller, Goerss and Hopkins, and in an alternative way by Lurie, see the survey [44. It is related to $K$-theory and String-bordism by a factorisation of the Witten genus

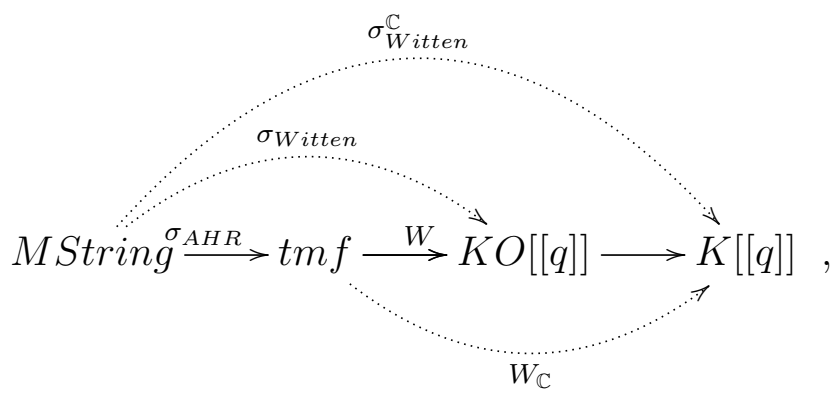


where $\sigma_{A H R}$ is the String-orientation of $\operatorname{tmf}$ constructed by Ando, Hopkins and Rezk. One of the interesting features of the restriction of $b^{a n}$ to the kernel of the map to Spinbordism is that it has a factorisation over $\sigma_{A H S}$. Since $b^{a n}$ is calculable in interesting cases it can be used to detect the $t m f$-class represented by a closed String-manifold.

Our goal here is Theorem 5.9 which gives the precise relation between $b^{\text {an }}$ and the universal $\eta$-invariant. In Corollary 5.12 we show one of the conjectures stated in [21 asserting that the factorisation of $b^{a n}$ over topological modular forms holds true on the whole Stringbordism group, i.e. we get rid of the restriction to the kernel to the Spin-bordism. Formally our proof is complete in dimensions $8 m-1$, while in dimensions $8 m-5$ we lose some two-torsion since in the present paper we work with complex $K$-theory instead of real $K$-theory. We strongly believe that the relevant part of the theory has a real version which does prove the case in dimension $8 m-5$ completely, too.

In Theorem 5.14 we show how the Riemannian geometry on a String-manifold together with a geometric string structure give rise to a geometrisation, and we derive the corresponding intrinsic formula for $b^{a n}$. We consider the proof of Theorem 5.14 as a model for many other situations where a construction of a geometrisation is required.

We start with recalling the definition of String-bordism. The homotopy type of the space BString is defined as a stage in the Whitehead tower of $B O$ :

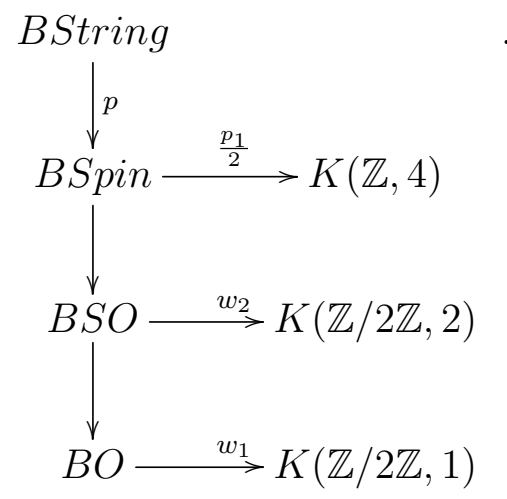

The space BString $=B O\langle 8\rangle$ is just a low instance of a whole tower of higher connected coverings $B O\langle n\rangle$ of the classifying space $B O$. Starting with BString these higher spaces are no longer associated to classical families of compact Lie groups.

In Lemma 5.13 we demonstrate that a connection on a Spin-principal bundle gives rise to a geometrisation. While the connection on the principal bundle allows to define connections on all associated vector bundles, the geometrisation partially keeps this information in terms of the differential $K$-theory classes represented by these vector bundles with connections. The geometrisation associated to a geometric String structure in this sense replaces the theory of connections on the non-existing principal bundle with structure group String. We think that the methods used in the case of BString $=B O\langle 8\rangle$ can easily be adapted to the higher stages $B O\langle n\rangle$.

We let MString be the Thom spectrum associated to the map BString $\rightarrow$ BSpin $\stackrel{\text { can }}{\rightarrow}$ BSpin ${ }^{c}$. The String-bordism spectrum MString is rationally even (see [37], [38], [36] for more calculations), so that for $m \in \mathbb{N}$ and $n=4 m-1$ the group $\pi_{n}$ (MString) is torsion. 
Hence the universal $\eta$-invariant gives a map

$$
\eta^{\text {top }}=\eta^{a n}: \pi_{n}(\text { MString }) \rightarrow Q_{n}(\text { MString }) .
$$

We will show that we can obtain $b^{a n}$ from the universal $\eta$-invariant by defining an interesting homomorphism out of the universal target $Q_{n}(M$ String). It involves evaluations against a collection of elements $R_{k}\left(\lambda^{\text {String }}\right) \in K^{0}$ (BString) for all $k \geq 0$. It is useful to organise this collection in a formal power series

$$
R\left(\lambda^{\text {String }}\right):=\sum_{k \geq 0} q^{k} R_{k}\left(\lambda^{\text {String }}\right) \in K[[q]]^{0}(B \text { String })
$$

which we will describe in the following. By $K[[q]]$ we denote the multiplicative cohomology theory (resp. the corresponding spectrum) which associates to a space $Y$ the ring

$$
K[[q]]^{*}(Y):=K^{*}(Y)[[q]]
$$

of formal power series with coefficients in $K^{*}(Y)$. The following constructions with real vector bundles are standard in the theory of the Witten genus (94), compare e.g. with [32, [21]. Given a real vector bundle $V \rightarrow Y$ we consider the element $R(V) \in K[[q]]^{0}(Y)$ defined by

$$
R(V):=\sum_{k=0}^{\infty} R_{k}(V) q^{k},
$$

where $R_{k}(V)$ is the $K$-theory class of the virtual bundle given by the coefficient in front of $q^{k}$ in the expansion of

$$
\prod_{k \geq 1}\left(1-q^{k}\right)^{2 \operatorname{dim}(V)} \bigotimes_{k \geq 1} \operatorname{Sym}_{q^{k}}\left(V \otimes_{\mathbb{R}} \mathbb{C}\right)
$$

where

$$
\operatorname{Sym}_{p}(V):=\bigoplus_{k \geq 0} p^{k} \operatorname{Sym}^{k}(V) .
$$

The transformation $V \mapsto R(V)$ is exponential, i.e. it satisfies $R(V \oplus W)=R(V) \cup R(W)$. Moreover, it has values in the group of multiplicative units $K[[q]]^{0}(Y)^{\times}$since the power series (91) starts with 1 . In view of the universal property of $K O^{0}$ it therefore extends to a natural transformation

$$
R: K O^{0}(Y) \rightarrow K[[q]]^{0}(Y)^{\times}
$$

The composition

$$
B \text { String } \rightarrow B O \stackrel{x \mapsto(0, x)}{\longrightarrow} \mathbb{Z} \times B O \cong \Omega^{\infty} K O
$$

classifies the universal class $\theta^{\text {String }} \in K O^{0}(B$ String $)$. We fix $n=4 m-1$ and let

$$
\lambda_{n}^{\text {String }}:=n-\theta^{\text {String }} \in K O^{0}(\text { BString }) .
$$


If $(M, f)$ is a cycle for $\pi_{n}(M S t r i n g)$, then we have the equality

$$
[T M]+1=f^{*} \lambda_{n+1}^{\text {String }}
$$

in $K O^{0}(M)$. We have well-defined classes $R_{k}\left(\lambda_{n+1}^{\text {String }}\right) \in K^{0}(B$ String $)$ for all $k \geq 0$ and therefore $R\left(\lambda_{n+1}^{\text {String }}\right) \in K[[q]]^{0}(B$ String $)$. With this notation the Witten genus

$$
\sigma_{W i t t e n}^{\mathbb{C}}: \pi_{n+1}(\text { MString }) \rightarrow \pi_{n+1}(K[[q]])
$$

satisfies

$$
\sigma_{W i t t e n}^{\mathbb{C}}(x)=\left\langle\operatorname{Thom}^{K}\left(R\left(\lambda_{n+1}^{\text {String }}\right)\right), \epsilon(x)\right\rangle .
$$

We use the superscript $\mathbb{C}$ in order to indicate that we work with the image of the $K O[[q]]$ valued Witten genus in complex $K$-theory $K[[q]]$.

We organize the evaluations of $Q_{n}(M$ String $)$ against the family of classes $R_{k}\left(\lambda_{n+1}^{\text {String }}\right)$ into a formal power series and define a homomorphism

$$
W: \operatorname{Hom}\left(K^{0}(\text { BString }), \pi_{n+1}(K \mathbb{Q} / \mathbb{Z})\right) \rightarrow \mathbb{Q} / \mathbb{Z}[[q]]:=\prod_{k \geq 0} \mathbb{Q} / \mathbb{Z} q^{k}
$$

by

$$
W(\phi):=\sum_{k \geq 0} \mathrm{ev}_{R_{k}\left(\lambda_{n+1}^{\text {String }}\right)}(\phi) q^{k} .
$$

The homomorphism (95) does not yet factorize over $Q_{n}($ MString) since it does not vanish on the subgroup $U^{\prime}$ appearing in (12). In order to get such a factorisation we must replace the target $\mathbb{Q} / \mathbb{Z}[[q]]$ of $W$ by the quotient by a subgroup which contains $W\left(U^{\prime}\right)$. This subgroup will be defined using modular forms. We let $\mathcal{M}_{2 m}^{R}$ denote the space of modular forms for $S L(2, \mathbb{Z})$ of weight $2 m$ whose $q$-expansion have coefficients in the subring $R \subseteq \mathbb{C}$ (see [32] for an introduction). In particular, we let

$$
\mathcal{M}_{2 m}^{\mathbb{Q}}[[q]] \subseteq \mathbb{Q}[[q]]
$$

be the finite-dimensional vector space of $q$-expansions of rational modular forms $\mathcal{M}_{2 m}^{\mathbb{Q}}$ of weight $2 m$. Its image in $\mathbb{Q} / \mathbb{Z}[[q]]$ will be denoted by $\overline{\mathcal{M}_{2 m}^{\mathbb{Q}}[[q]]}$. We define

$$
T_{2 m}:=\frac{\mathbb{Q} / \mathbb{Z}[[q]]}{\overline{\mathcal{M}_{2 m}^{\mathbb{Q}}[[q]]}} .
$$

Up to the replacement of $\mathbb{Q}$ by $\mathbb{R}$ this is exactly the group defined in [21, Def. 1.1].

Lemma 5.8 The composition of (95) with the projection to the quotient 96) induces a well-defined map

$$
\bar{W}: Q_{4 m-1}(\text { MString }) \rightarrow T_{2 m} .
$$


Proof. We must show that under this composition the subgroup $U$ defined in (5) is mapped to $\overline{\mathcal{M}_{2 m}^{\mathbb{Q}}[[q]]}$. By 94 we have for $y \in \pi_{n+1}(M$ String $)$ that

$$
\left\langle\operatorname{Thom}^{K}\left(R\left(\lambda_{n+1}^{\text {String }}\right)\right), \epsilon(y)\right\rangle=\sigma_{W i t t e n}^{\mathbb{C}}(y) \in \pi_{n+1}(K[[q]]) \cong \mathbb{Z}[[q]] .
$$

We now use the fact that the Witten genus has values in $\mathcal{M}_{2 m}^{\mathbb{Z}}[[q]] \subset \mathbb{Z}[[q]]$. More generally, for $y \in \pi_{n+1}(M$ String $\mathbb{Q})$ we get

$$
\left\langle\operatorname{Thom}^{K}\left(R\left(\lambda_{n+1}^{\text {String }}\right)\right), \epsilon(y)\right\rangle \in \mathcal{M}_{2 m}^{\mathbb{Q}}[[q]] .
$$

This shows that $\bar{W}\left(U^{\prime}\right) \subseteq \overline{\mathcal{M}_{2 m}^{\mathbb{Q}}[[q]]}$.

In [21, Sec 3.3] we have constructed homomorphisms

$$
b^{a n}: \pi_{4 m-1}(M \text { String }) \rightarrow T_{2 m}, \quad b^{\text {top }}: A_{4 m-1} \rightarrow T_{2 m}
$$

where

$$
A_{4 m-1}:=\operatorname{ker}\left(\pi_{4 m-1}(\text { MString }) \rightarrow \pi_{4 m-1}(\text { MSpin })\right) .
$$

Since in the present paper we work we complex $K$-theory as opposed to real $K$-theory in [21, Sec 3.3] we define

$$
b_{\mathbb{C}}^{*}:=\left\{\begin{array}{cc}
b^{*} & \text { m even } \\
2 b^{*} & \text { modd }
\end{array} \quad, \quad * \in\{\text { an, top }, \text { tmf }\} .\right.
$$

Theorem 5.9 We have the equalities

$$
\bar{W} \circ \eta_{\mid A_{4 m-1}}^{\text {top }}=b_{\mathbb{C}}^{\text {top }}
$$

and

$$
\bar{W} \circ \eta^{a n}=b_{\mathbb{C}}^{a n}
$$

Proof. We extend the map MString $\rightarrow$ MSpin to a fibre sequence

$$
\Sigma^{-1} \text { MSpin } \rightarrow \mathcal{A} \rightarrow \text { MString } \rightarrow \text { MSpin }
$$

which defines the spectrum $\mathcal{A}$. The smash product of the fibre sequence with the fibre sequence (2) yields the following quadratic diagram

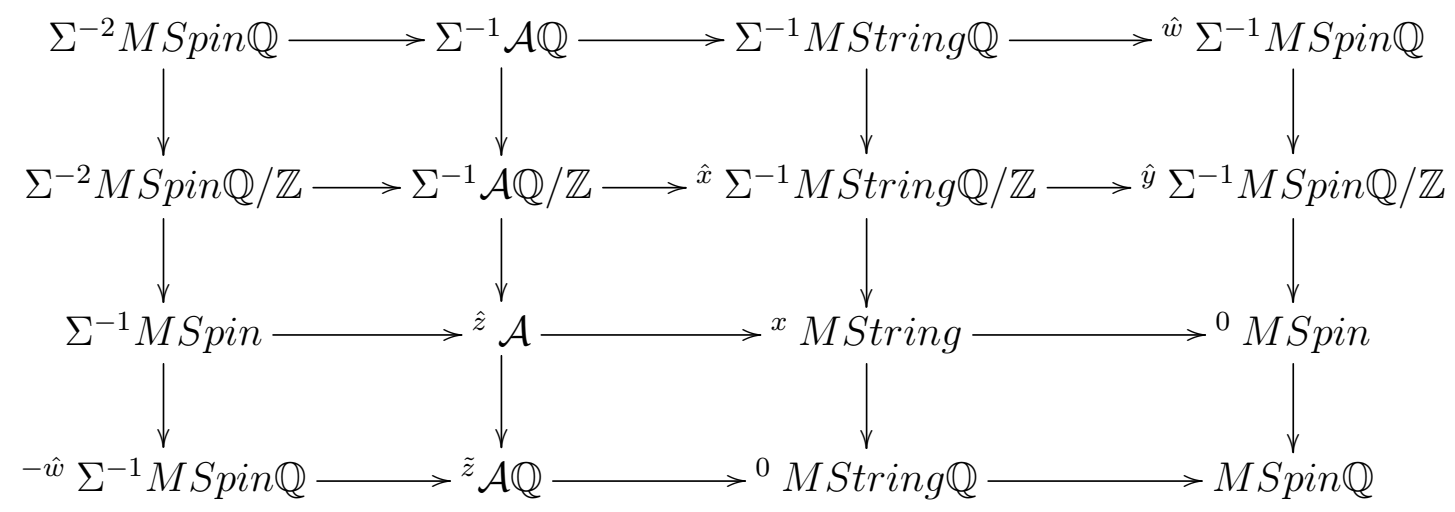


We start with $x \in A_{4 m-1} \subseteq \pi_{4 m-1}(M$ String). This element goes to zero if it is mapped to the right or down. The class $\bar{W}\left(\eta^{t o p}(x)\right)$ is represented by the power series

$$
\sum_{k \geq 0}\left\langle\operatorname{Thom}^{K}\left(R_{k}\left(\lambda_{4 m}^{\text {String }}\right)\right), \epsilon(\hat{x})\right\rangle q^{k} \in \mathbb{Q} / \mathbb{Z}[[q]]
$$

Note that we can define classes $\theta^{\text {Spin }}$ and $\lambda_{n}^{\text {Spin }}:=n-\theta^{\text {Spin }} \in K O^{0}($ B Spin $)$ analogously to $(92)$. Then we have equalities of the evaluations

$$
\begin{aligned}
\left\langle\operatorname{Thom}^{K}\left(R_{k}\left(\lambda_{4 m}^{\text {String }}\right)\right), \epsilon(\hat{x})\right\rangle & =\left\langle\operatorname{Thom}^{K}\left(R_{k}\left(\lambda_{4 m}^{\text {Spin }}\right)\right), \epsilon(\hat{y})\right\rangle \\
& =\left[\left\langle\operatorname{Thom}^{K}\left(R_{k}\left(\lambda_{4 m}^{\text {Spin }}\right)\right), \epsilon(\hat{w})\right\rangle\right] \in \mathbb{Q} / \mathbb{Z},
\end{aligned}
$$

where the elements $\hat{y}$ and $\hat{w}$ are images and lifts as indicated in the above diagram, and where we use the compatibility of the $K$-theory Thom isomorphisms for MString and MSpin. In the construction of $b^{\text {top }}$ in [21, Sec 4.1] we go the other way. We first lift $x$ to $\hat{z}$ which maps to $\tilde{z}$ which is then again lifted to $\Sigma^{-1} M \operatorname{Spin} \mathbb{Q}$. It is a general fact of such a diagram chase in a product of fibre sequences that modulo the obvious ambiguities this element in the lower left corner is the negative of $\hat{w}$ from the upper right corner. By the definition of $b^{\text {top }}$ in [21, Sec 4.1$]$ we see that $b_{\mathbb{C}}^{\text {top }}(x)$ is represented by

$$
\sum_{k \geq 0}\left[\left\langle\operatorname{Thom}^{K}\left(R_{k}\left(\lambda_{4 m}^{\text {Spin }}\right)\right), \epsilon(\hat{w})\right\rangle\right] q^{k} \in \mathbb{Q} / \mathbb{Z}[[q]]
$$

Combining this with 98 and 99 we see that

$$
\bar{W} \circ \eta_{\mid A_{4 m-1}}^{t o p}=b_{\mathbb{C}}^{t o p} .
$$

This proves the first assertion of Theorem 5.9 .

We now show the second. Let $x=[M, f] \in \pi_{n}$ (MString) be an l-torsion element represented by the cycle $(M, f)$ and $(W, F)$ be a zero-bordism of the union of $l$ copies of $(M, f)$. The Spin ${ }^{c}$-structures come from Spin-structures so that the Levi-Civita connections have canonical $S p i n^{c}$-extensions $\tilde{\nabla}^{T M}$ and $\tilde{\nabla}^{T W}$. In view of Equation (93) the class $f^{*} R\left(\lambda_{4 m}^{\text {String }}\right) \in K[[q]]^{0}(M)$ can be represented by a formal power series of $\mathbb{Z} / 2 \mathbb{Z}$-graded bundles $R(T M \oplus 1)$ associated to the tangent bundle. The Riemannian metric and the Levi-Civita connection turn $T M$ into a geometric bundle. The construction of $R(T M \oplus 1)$ therefore produces a formal power series of geometric bundles $\mathbf{R}(T M \oplus 1)$.

The construction of $b^{a n}$ involves the choice of a geometric String-structure $\alpha$ on $M$. This notion has been introduced in [53]. In particular, a geometric string structure produces a form $H_{\alpha} \in \Omega^{3}(M)$ with the property that

$$
2 d H_{\alpha}=p_{1}\left(\nabla^{T M, L C}\right) .
$$

In the following we use characteristic forms associated to certain power series

$$
\tilde{\Phi}, \Phi, \Theta \in \mathbb{Q}[[q]]\left[b, b^{-1}\right]\left[\left[b^{2} p_{1}, b^{4} p_{2}, \ldots\right]\right] \text {. }
$$


We refer to [21, Sec 3.3] or equation (108) for an explicit definition. In the notation of the latter we have

$$
\Phi:=\Phi_{R\left(\lambda_{4 m}^{\text {Spin }}\right)}, \quad \tilde{\Phi}:=\tilde{\Phi}_{R\left(\lambda_{4 m}^{\text {Spin }}\right)}, \quad \Theta:=\Phi-p_{1} \tilde{\Phi} .
$$

In the present paper we distribute the powers of $b$ such that $\Phi$ and $\Theta$ have total degree zero, and $\tilde{\Phi}$ has total degree -4 . The notation $\tilde{\Phi}\left(\tilde{\nabla}^{T M}\right)$ is as in 109 . We start with the representative of $b_{\mathbb{C}}^{a n}(x)$ given in [21, Def 4.1]

$$
\left.\left[2 \int_{M} H_{\alpha} \wedge \tilde{\Phi}\left(\tilde{\nabla}^{T M}\right)\right]-\xi\left(\not D_{M} \otimes \mathbf{R}(T M \oplus 1)\right)\right] \in \mathbb{R} / \mathbb{Z}[[q]]
$$

where here and below we ignore the power $b^{2 m}$. We use the APS index formula $(28)$ in order to express the reduced $\eta$-invariant appearing in (101). Using the equality

$$
\Phi\left(\tilde{\nabla}^{T W}\right)=\mathbf{T d}\left(\tilde{\nabla}^{T M}\right) \wedge \mathbf{c h}\left(\nabla^{\mathbf{R}(T M \oplus 1)}\right)
$$

we get

$$
101)=\left[2 \int_{M} H_{\alpha} \wedge \tilde{\Phi}\left(\tilde{\nabla}^{T M}\right)-\frac{1}{l} \int_{W} \Phi\left(\tilde{\nabla}^{T W}\right)\right]+\left[\frac{1}{l} \operatorname{index}\left(\not D_{W} \otimes \mathbf{R}(T W)\right)_{A P S}\right] .
$$

We now use Stokes' theorem and the relation 100 in order to calculate

$$
\begin{aligned}
2 \int_{M} H_{\alpha} & \wedge \tilde{\Phi}\left(\tilde{\nabla}^{T M}\right)-\frac{1}{l} \int_{W} \Phi\left(\tilde{\nabla}^{T W}\right) \\
& =\frac{1}{l} \int_{W}\left(p_{1}\left(\nabla^{T W}\right) \wedge \tilde{\Phi}\left(\tilde{\nabla}^{T M}\right)-\Phi\left(\tilde{\nabla}^{T W}\right)\right) \\
& =\frac{1}{l} \int_{W} \Theta\left(\tilde{\nabla}^{T W}\right) \in \mathcal{M}_{2 m}^{\mathbb{R}}[[q]] .
\end{aligned}
$$

For the last inclusion we use the crucial fact that

$$
p_{4 m}(\Theta) \in \mathcal{M}_{2 m}^{\mathbb{Q}}[[q]]\left[b^{2} p_{1}, b^{4} p_{2}, \ldots\right] \subset \mathbb{Q}[[q]]\left[\left[b^{2} p_{1}, b^{4} p_{2}, \ldots\right]\right],
$$

see [21, Sec. 3.3]. Therefore

$$
\left[\frac{1}{l} \operatorname{index}\left(\not D_{W} \otimes \mathbf{R}(T W)\right)_{A P S}\right] \in \mathbb{R} / \mathbb{Z}[[q]]
$$

is a representative of $b_{\mathbb{C}}^{a n}(x) \in T_{2 m}$, too. But in view of Definition 3.5 and the construction of $\bar{W}$ this is also a representative of $\bar{W}\left(\eta^{a n}(x)\right) \in T_{2 m}$. This shows

$$
\bar{W} \circ \eta^{a n}=b_{\mathbb{C}}^{a n} .
$$

As a consequence of the equality $\eta^{a n}=\eta^{t o p}$ shown in Theorem 3.6 we get another proof of [21, Thm. 2.2]. 
Corollary 5.10 We have the equality

$$
b_{\mathbb{C} \mid A_{4 m-1}}^{a n}=b_{\mathbb{C}}^{\text {top }} .
$$

We now recall from [21, Sec.4.3] the construction of the homomorphism

$$
b^{t m f}: \pi_{4 m-1}(t m f) \rightarrow T_{2 m}
$$

which is very similar to that of $\eta^{t o p}$. Note that $\pi_{4 m-1}(t m f)$ is a torsion group (see [34], 12] for more calculations of $\left.\pi_{*}(t m f)\right)$. Therefore an element $y \in \pi_{4 m-1}(t m f)$ can be lifted to an element $\hat{y} \in \pi_{4 m}(\operatorname{tmf} \mathbb{Q} / \mathbb{Z})$. Then

$$
b^{t m f}(y):=[W(\hat{y})] \in T_{2 m},
$$

where $[W(\hat{y})]$ denotes the class in $T_{2 m}$ of the element $W(\hat{y}) \in \pi_{4 m}(K O[[q]] \mathbb{Q} / \mathbb{Z}) \cong \mathbb{Q} / \mathbb{Z}[[q]]$ and $W$ is as in $(90)$. The complex version $b_{\mathbb{C}}^{\text {tmf }}$ of $b^{\text {tmf }}$ is defined similarly by

$$
b_{\mathbb{C}}^{t m f}(y):=\left[W_{\mathbb{C}}(\hat{y})\right] \in T_{2 m},
$$

or alternatively, by (97).

Proposition 5.11 We have the equality

$$
b_{\mathbb{C}}^{t m f} \circ \sigma_{A H R}=\bar{W} \circ \eta^{t o p}: \pi_{4 m-1}(\text { MString }) \rightarrow T_{2 m} .
$$

Proof. If $x \in \pi_{4 m-1}(M$ String $)$ and $\hat{x} \in \pi_{4 m}(M$ String $\mathbb{Q} / \mathbb{Z})$ is a lift, then

$$
\left\langle\operatorname{Thom}^{K}\left(R\left(\lambda_{4 m}^{\text {String }}\right)\right), \epsilon(\hat{x})\right\rangle \in \mathbb{Q} / \mathbb{Z}[[q]]
$$

represents $\bar{W} \circ \eta^{t o p}(x)$. We have already seen in the proof of Lemma 5.8 that this expression is equal to the Witten genus (extended to $\mathbb{Q} / \mathbb{Z}$-theory)

$$
\left\langle\operatorname{Thom}^{K}\left(R\left(\lambda_{4 m}^{\text {String }}\right)\right), \epsilon(\hat{x})\right\rangle=\sigma_{W i t t e n}^{\mathbb{C}}(\hat{x}) .
$$

The Witten genus (see (90) ) can now be decomposed as

$$
\sigma_{W i t t e n}^{\mathbb{C}}(\hat{x})=W_{\mathbb{C}}\left(\sigma_{A H R}(\hat{x})\right)
$$

We can take $\sigma_{A H R}(\hat{x}) \in \pi_{4 m}(t m f \mathbb{Q} / \mathbb{Z})$ as the lift of $\sigma_{A H R}(x) \in \pi_{4 m}(t m f)$ so that $W_{\mathbb{C}}\left(\sigma_{A H R}(\hat{x})\right)$ represents $b_{\mathbb{C}}^{t m f}\left(\sigma_{A H R}(x)\right)$. Hence we can conclude that

$$
b_{\mathbb{C}}^{t m f} \circ \sigma_{A H R}(x)=\bar{W} \circ \eta^{t o p}(x) .
$$

Using Theorem 3.6 stating the equality $\eta^{a n}=\eta^{t o p}$ and $\bar{W} \circ \eta^{a n}=b_{\mathbb{C}}^{a n}$ given by Theorem 5.9 we get 
Corollary 5.12 We have

$$
b_{\mathbb{C}}^{a n}=b_{\mathbb{C}}^{t m f} \circ \sigma_{A H R} .
$$

This proves the complex version of the conjecture 3 in [21, Sec 1.5]. In fact, for even $m$ there is no difference between the real and complex case, but in the case of odd $m$ the complex version implies the real version up to two-torsion which was known before. We believe that a real version of the present theory would prove the conjecture completely.

The formula for $b^{a n}$ given in [21, Sec 3.3] and reproduced here as (101) is an intrinsic formula which uses the notion of a geometric String-structure [53]. In the following we show that a geometric String-structure gives rise to a good geometrisation $\mathcal{G}^{\text {String }}$ of $\left(M, f, \tilde{\nabla}^{T M}\right)$ such that the intrinsic formula Theorem 4.19 specialises to the one for $b^{a n}$. Since String-structures refine Spin-structures we start with the construction of a geometrisation for a Spin-structure.

We consider a cycle $(M, f)$ for $\pi_{n}(M S p i n)$. We are going to use a version of Subsection 3.3 for Spin-structures. If $V \rightarrow M$ is a real euclidean oriented vector bundle, then the $\operatorname{Spin}$-gerbe $\operatorname{Spin}(V)$ of $V$ associates to each open subset $A \subseteq M$ the groupoid $\operatorname{Spin}\left(V_{\mid A}\right)$ of Spin-structures on the restriction of $V$ to $A$. This gerbe has the band $\mathbb{Z} / 2 \mathbb{Z}$, and its isomorphism class is classified by the Dixmier-Douady class

$$
\mathrm{DD}(\underline{\operatorname{Spin}(V)})=w_{2}(V) \in H^{2}(M ; \mathbb{Z} / 2 \mathbb{Z}),
$$

the second Stiefel-Whitney class of $V$.

We choose a tangential Spin-structure on $T M$ given by a geometric Spin-structure $P \in$ $\operatorname{Spin}(T M)$ together with a trivialisation

$$
P \otimes \tilde{f}^{*} Q_{k}^{S p i n} \cong Q(n+k)
$$

where $\tilde{f}: M \rightarrow B \operatorname{Spin}(k)$ is some factorisation of $f$, and $Q_{k}^{\operatorname{Spin}} \rightarrow B \operatorname{Spin}(k)$ denotes the universal Spin-bundle. It naturally induces a tangential $S$ in $^{c}$-structure by extension of structure groups along $\operatorname{Spin}(l) \rightarrow \operatorname{Spin}^{c}(l)$ (see [43, Example D.5]).

The Levi-Civita connection gives rise to a connection $\nabla^{T M}$ on $P$ which in turn has a natural $S_{p i n}^{c}$-extension $\tilde{\nabla}^{T M}$. We furthermore choose a connection $\nabla^{k}:=\nabla^{\tilde{f}^{*} Q_{k}^{S p i n}}$ on $\tilde{f}^{*} Q_{k}^{S p i n}$ and let $\tilde{\nabla}^{k}$ be its Spin $^{c}$-extension.

Proposition 5.13 There exists a geometrisation $\mathcal{G}^{\text {Spin }}$ of $\left(M, f, \tilde{\nabla}^{T M}\right)$ which is $\ell$-good for every $\ell \in \mathbb{N}$.

Proof. The connections $\tilde{\nabla}^{T M}$ and $\tilde{\nabla}^{k}$ together induce a $S p i n^{c}$-extension $\tilde{\nabla}^{T M} \otimes \tilde{\nabla}^{k}$ of the connection $\nabla^{T M} \otimes \nabla^{k}$ on $P \otimes \tilde{f}^{*} Q_{k}^{S p i n}$ which can be compared with a trivial connection using the isomorphism 102 . Therefore the transgression form $\tilde{\mathbf{T}} \mathbf{d}\left(\tilde{\nabla}^{T M} \otimes \tilde{\nabla}^{k}, \nabla^{\text {triv }}\right) \in$ $\Omega P^{-1}(M) / \operatorname{im}(d)$ is defined and satisfies

$$
d \tilde{\mathbf{T}} \mathbf{d}\left(\tilde{\nabla}^{T M} \otimes \tilde{\nabla}^{k}, \nabla^{\text {triv }}\right)=\mathbf{T} \mathbf{d}\left(\tilde{\nabla}^{T M}\right) \wedge \mathbf{T} \mathbf{d}\left(\tilde{\nabla}^{k}\right)-1 .
$$

We let

$$
\mu:=\mathbf{T d}\left(\tilde{\nabla}^{k}\right)^{-1} \wedge \tilde{\mathbf{T}} \tilde{\mathbf{d}}\left(\nabla^{T M} \otimes \tilde{\nabla}^{k}, \nabla^{\text {triv }}\right)
$$


For any space or spectrum $Y$ we let $\bar{K}^{*}(Y)$ denote the completion the topological group $K^{*}(Y)$ equipped with the profinite topology (see [16, Def. 4.9] or Remark 2.1). We have $B \operatorname{Spin}:=\operatorname{hocolim}_{l} B \operatorname{Spin}(l)$ and therefore $\bar{K}^{*}(B \operatorname{Spin}) \cong \lim _{l} \bar{K}^{*}(B \operatorname{Spin}(l))$. The completion theorem [8] gives

$$
K^{*}(B \operatorname{Spin}(k))=\bar{K}^{*}(B \operatorname{Spin}(k)) \cong R\left(\operatorname{Spin}(k) \hat{)}_{I_{\operatorname{Spin}(k)}} .\right.
$$

We therefore get the following description of the completion of the $K$-theory of BSpin:

$$
\bar{K}^{*}(B \operatorname{Spin}) \cong \lim _{l} K^{*}(B \operatorname{Spin}(k+l)) \cong \lim _{l} R\left(\operatorname{Spin}(k+l){\hat{I_{S p i n}(k+l)}} .\right.
$$

We fix an integer $l \geq 0$ and form the $l$-fold stabilization $\tilde{f}^{*} Q_{k}^{S p i n} \otimes Q(l)$ of $\tilde{f}^{*} Q_{k}^{S p i n}$. This stabilization is a $\operatorname{Spin}(k+l)$-principal bundle with the connection $\nabla^{k} \otimes \nabla^{Q(l)}$. Given a representation $\rho$ of $\operatorname{Spin}(k+l)$ we define a geometric bundle $\mathbf{V}_{\rho}$ as an associated bundle to $\tilde{f}^{*} Q_{k}^{S p i n} \otimes Q(l)$. We define

$$
G_{l}(\rho):=\left[\mathbf{V}_{\rho}\right]-a\left(\mu \wedge \operatorname{ch}\left(\nabla^{V_{\rho}}\right)\right) \in \hat{K}^{0}(M) .
$$

We have chosen the form $\mu$ in 103 such that the following equality holds true in $\Omega P_{c l}^{0}(M)$ :

$$
\operatorname{Td}\left(\tilde{\nabla}^{T M}\right) \wedge R(G(\rho))=\operatorname{Td}\left(\tilde{\nabla}^{k}\right)^{-1} \wedge \operatorname{ch}\left(\nabla^{V_{\rho}}\right) .
$$

The map $\rho \mapsto G_{l}(\rho)$ extends to a map $G_{l}: R(\operatorname{Spin}(k+l)) \rightarrow \hat{K}^{0}(M)$ by linearity. This extension annihilates the $2 n+1$ 'th power $I_{\operatorname{Spin}(k+l)}^{2 n+1} \subseteq R(\operatorname{Spin}(k+l))$ of the dimension ideal. In order to see this note that if $\rho \in I_{\operatorname{Spin}(k+l)}^{p}$ and $2 p>n$, then we have $\mathbf{c h}\left(\nabla^{V_{\rho}}\right)=0$. For those $\rho$ we have $G_{l}(\rho)=\left[\mathbf{V}_{\rho}\right]$, and this class is flat. If $p>n$, then we have $\left[V_{\rho}\right]=0$ so that $G_{l}(\rho)=a(\omega)$ for some $\omega \in H P \mathbb{R}^{-1}(M)$. The product of a flat class with a class of this form vanishes by (47). Hence $G_{l}(\rho)=0$ if $p>2 n$. The map $G_{l}$ thus further extends be continuity to a map

$$
\mathcal{G}^{\operatorname{Spin}(k+l)}: K^{0}(B \operatorname{Spin}(k+l)) \rightarrow \hat{K}^{0}(M)
$$

One now checks that for $l \geq 1$ we have

$$
G_{l}(\rho)=G_{l-1}\left(\rho_{\mid \operatorname{Spin}(k+l-1}\right)
$$

In this way the maps $\mathcal{G}^{\operatorname{Spin}(k+l)}$ for the various $l$ are compatible. We consider the continuous map

$$
\mathcal{G}^{\text {Spin }}: \bar{K}^{0}(B \operatorname{Spin}) \rightarrow K^{0}(B \operatorname{Spin}(k)) \stackrel{\mathcal{G}^{\operatorname{Spin}(k)}}{\rightarrow} \hat{K}^{0}(M)
$$

We now show that $\mathcal{G}^{\text {Spin }}$ and $\mathcal{G}^{\text {Spin }(k+l)}$ are geometrisations. To this end we must show that they admit degree-perserving cohomological characters. By their compatibility it suffices to consider $\mathcal{G}^{\operatorname{Spin}(k)}$. It follows from 104 and $\mathbf{T d}\left(\tilde{\nabla}^{T M}\right)=\hat{\mathbf{A}}\left(\nabla^{T M}\right)$ (since $\tilde{\nabla}^{T M}$ is induced from a Spin-connection $\left.\nabla^{T M}\right)$ that $\mathbf{T} \mathbf{d}\left(\tilde{\nabla}^{T M}\right) \wedge R\left(G_{0}(\rho)\right)$ is the Chern-Weyl 
representative of the class $\hat{\mathbf{A}}^{-1} \cup \mathbf{c h}([\rho]) \in H P \mathbb{Q}^{0}(B \operatorname{Spin}(k))$ associated to the connection $\nabla^{k}$, where $[\rho] \in K^{0}(B \operatorname{Spin}(k))$ is the class represented by $\rho$. Note that

$$
H^{*}(\operatorname{BSpin}(k) ; \mathbb{Q}) \cong \mathbb{Q}\left[p_{1}, p_{2}, \ldots, p_{r_{k}}\right]
$$

is the polynomial ring generated by the universal Pontrjagin classes. For the cohomological character

$$
c_{\mathcal{G}^{\operatorname{Spin}(k)}}: H P \mathbb{Q}^{0}(\operatorname{BSpin}(k)) \rightarrow \Omega P_{c l}^{0}(M)
$$

of $\mathcal{G}^{\operatorname{Spin}(k)}$ we choose the map which sends the class $b^{2 i} p_{i} \in H P \mathbb{Q}^{0}(B \operatorname{Spin}(k))$ to the corresponding characteristic form $b^{2 i} p_{i}\left(\nabla^{k}\right) \in \Omega P_{c l}^{0}(M)$. This map clearly preserves degrees.

We now show that $\mathcal{G}^{\text {Spin }}$ is $\ell$-good for every $\ell \in \mathbb{N}$. By an inspection of the construction of $\mathcal{G}^{\text {Spin }}$ we observe that the connection of the map $f: M \rightarrow B$ with the stable normal bundle of $M$ has not been used. This map can be arbitrary if we replace $T M$ by some complement $\eta \rightarrow M$ of $\hat{f}^{*} \xi_{k}$ as in Subsection 4.3 and choose some connection $\nabla^{\eta}$ of the associated complementary Spin-principal bundle $P^{\operatorname{Spin}} \in \operatorname{Spin}(\eta)$ in the place of $\nabla^{T M}$. We obtain the $\operatorname{Spin}^{c}$-bundle $P$ with connection $\tilde{\nabla}^{\eta}$ which replaces $\tilde{\nabla}^{T M}$ by extension of the structure group.

Let $\ell \in \mathbb{N}$ be such that $\ell \geq n+1$. We choose an $\ell$-connected approximation $f_{u}: M_{u} \rightarrow$ BSpin such that there is a factorisation of $f$ over a closed embedding $h: M \rightarrow M_{u}$. As in Subsection 4.4 we obtain a natural refinement of $h$ to a $\operatorname{Spin}^{c}$-map. Since $h$ is a closed embedding we can choose the connections $\tilde{\nabla}^{u}$ on $P_{u}$ and $\nabla^{k, u}$ on $\tilde{f}_{u}^{*} Q_{k}^{S p i n}$ such that $h^{*} \tilde{\nabla}^{u}=\tilde{\nabla}^{T M}$ stably and $h^{*} \nabla^{k, u}=\nabla^{k}$. We now define $\mathcal{G}_{u}^{\text {Spin }}$ as above. Then by construction $\mathcal{G}^{\text {Spin }}=h^{*} \mathcal{G}_{u}^{\text {Spin }}$ since the correction forms 58 vanishes. Since $\ell$ can be taken arbitrary large we see that $\mathcal{G}^{S p i n}$ is $\ell$-good for every $\ell \in \mathbb{N}$.

Note that the geometrizations $\mathcal{G}^{\operatorname{Spin}(k+l)}$ constructed in the proof of Proposition 5.13 are $\ell$-good for every $\ell \in \mathbb{N}$, too.

Let $p: B$ String $\rightarrow$ BSpin be the natural map. We consider a cycle $(M, f)$ for $\pi_{n}(M$ String $)$. Then $(M, p \circ f)$ naturally becomes a cycle for $\pi_{n}(M \operatorname{Spin})$.

Theorem 5.14 A choice of a geometric string structure $\alpha$ on $\left(\tilde{f}^{*} Q_{k}^{\text {Spin }}, \nabla^{k}\right)$ naturally determines a geometrisation $\mathcal{G}^{\text {String }}$ of $\left(M, f, \tilde{\nabla}^{T M}\right)$ which is $\ell$-good for every $\ell \in \mathbb{N}$. For $\phi \in \bar{K}^{0}(B$ Spin $)$ it is given by

$$
\mathcal{G}^{\text {String }}\left(p^{*} \phi\right)=\mathcal{G}^{\text {Spin }}(\phi)-a\left(\nu_{\phi}\right)
$$

with

$$
\nu_{\phi}:=\mathbf{T d}\left(\tilde{\nabla}^{T M}\right)^{-1} \wedge 2 H_{\alpha} \wedge \tilde{\Phi}_{\phi}\left(\nabla^{m}\right) \in \Omega P^{-1}(M) .
$$

Here $\mathcal{G}^{\text {Spin }}$ is as in Lemma 5.13, $\tilde{\Phi}_{\phi}\left(\nabla^{m}\right)$ is defined below in (109), and $H_{\alpha} \in \Omega^{3}(M)$ is the three-form given by the geometric string structure. 
Proof. We fix an integer $\ell \geq n+1$. For a sufficiently large integer $m \in \mathbb{N}, m \geq 3$ we can assume that the map $f$ has a factorization

$$
M \stackrel{f_{m}}{\rightarrow} B \operatorname{String}(m) \stackrel{\iota_{m}}{\rightarrow} \text { BString }
$$

such that $\iota_{m}$ is $\ell$-connected. We have a fibre sequence

$$
K(\mathbb{Z}, 3) \rightarrow B \operatorname{String}(m) \stackrel{p}{\rightarrow} B \operatorname{Spin}(m) \rightarrow K(\mathbb{Z}, 4)
$$

By [4] the reduced $K$-theory group $\tilde{K}^{*}(K(\mathbb{Z}, 3))$ is uniquely divisible and consists of phantom classes, i.e. classes which vanish when pulled-back to finite $C W$-complexes. This suggests the following proposition which is probably well-known, but we could not find a reference for it.

Proposition 5.15 For $m \geq 3$ the pull-back

$$
p^{*}: \bar{K}^{*}(B \operatorname{Spin}(m)) \rightarrow \bar{K}^{*}(B \operatorname{String}(m))
$$

along the projection $p: B \operatorname{String}(m) \rightarrow B \operatorname{Spin}(m)$ represents $\bar{K}^{*}(B \operatorname{String}(m))$ as a completion of $\bar{K}^{*}(B \operatorname{Spin}(m))$ with respect to the topology induced on $\bar{K}^{*}(B \operatorname{Spin}(m))$ via $p^{*}$ by the profinite topology of $\bar{K}^{*}(B$ String $(m))$.

We defer the proof of this proposition to Section 6. and continue with the construction of a geometrisation $\mathcal{G}^{\text {String }}$ which is $\ell$-good. We choose an $\ell+1$-connected approximation $f_{u}: M_{u} \rightarrow B \operatorname{String}(m)$ such that we can factorize $f_{m}: M \rightarrow B \operatorname{String}(m)$ over the closed embedding $h: M \rightarrow M_{u}$. As in Subsection 4.4 we obtain a natural refinement of $h$ to a $\operatorname{Spin}^{c}$-map. Since $h$ is a closed embedding we can choose the connections $\tilde{\nabla}^{u}$ on $P_{u}$ and $\nabla^{m, u}$ on $f_{u}^{*} Q_{m}^{\text {Spin }}$ such that $h^{*} \tilde{\nabla}^{u}=\tilde{\nabla}^{T M}$ stably and $h^{*} \nabla^{m, u}=\nabla^{m}$. Geometric string structures behave as flexible as connections and metrics [53]. We can therefore assume that there is a geometric string structure $\alpha_{u}$ on $\left(f_{u}^{*} Q_{m}^{S p i n}, \nabla^{m, u}\right)$ which restricts to the geometric string structure $\alpha$ on $M$.

In the proof of Lemma 5.13 we have constructed a geometrisation

$$
\mathcal{G}_{u}^{\operatorname{Spin}(m)}: \bar{K}^{0}(B \operatorname{Spin}(m)) \rightarrow \hat{K}^{0}\left(M_{u}\right)
$$

of $\left(M_{u}, p \circ f_{u}, \nabla^{m, u}\right)$. As a first approximation we define

$$
\mathcal{G}_{u, 0}^{\text {String }(m)}: \bar{K}^{0}(B \operatorname{Spin}(m)) \rightarrow \hat{K}^{0}\left(M_{u}\right)
$$

by

$$
\mathcal{G}_{u, 0}^{\operatorname{String}(m)}(\phi):=\mathcal{G}_{u}^{\operatorname{Spin}(m)}(\phi), \quad \phi \in \bar{K}^{0}(\operatorname{BSpin}(m)) .
$$

In view of Proposition 5.15 the homomorphism $\mathcal{G}_{u, 0}^{\text {String }(m)}$ is defined on a group which defines $\bar{K}^{0}(B$ String $(m))$ by completion with respect to a certain topology. If $\mathcal{G}_{u, 0}^{\text {String }(m)}$ would be continuous with respect to this topology it would descend to a continuous 
homomorphism defined on $\bar{K}^{0}(B \operatorname{String}(m))$. Note that $\mathcal{G}_{u, 0}^{\text {String }(m)}$ is not continuous and the idea is now to add a correction term in order to improve the continuity.

Note that via $p^{*}$ we can identify

$$
H \mathbb{Q}^{*}(B \operatorname{String}(m) ; \mathbb{Q}) \cong \mathbb{Q}\left[\left[p_{2}, \ldots, p_{r_{m}}\right]\right]
$$

with the quotient ring of $H^{*}(B \operatorname{Spin}(m) ; \mathbb{Q})$ given in 105$)$ by setting $p_{1}=0$. The problem with continuity comes from the contribution of $p_{1}\left(\tilde{\nabla}^{u}\right)$ to the curvature of $\mathcal{G}_{u, 0}^{\operatorname{String}(m)}$. The idea is now to kill this contribution by a correction term given by a geometric string structure $\alpha_{u}$ on $\left(\tilde{f}_{u}^{*} Q_{m}^{\text {Spin }}, \nabla^{m, u}\right)$. The geometric string structure provides the form $H_{\alpha_{u}} \in$ $\Omega^{3}\left(M_{u}\right)$ with the property that $2 d H_{\alpha_{u}}=p_{1}\left(\nabla^{m, u}\right)$ (see 100).

For a formal power series

$$
\Lambda \in \mathbb{Q}\left[b, b^{-1}\right]\left[\left[b^{2} p_{1}, b^{4} p_{2}, \ldots\right]\right]
$$

we define a new formal power series

$$
\tilde{\Lambda}:=\frac{\Lambda-i_{p_{1}=0} \Lambda}{p_{1}} \in \mathbb{Q}\left[b, b^{-1}\right]\left[\left[b^{2} p_{1}, b^{4} p_{2}, \ldots\right]\right] .
$$

In other words, the power series $\tilde{\Lambda}$ is $p_{1}^{-1}$ times the sum of those monomials of $\Lambda$ which contain $p_{1}$. Since the periodic rational cohomology of any space $Y$ is complete, i.e. we have $H P \mathbb{Q}^{*}(Y) \cong{\overline{H P \mathbb{Q}^{*}}}^{*}(Y)$, the Chern character factorizes over the completion of $K$ theory as ch : $\bar{K}^{0}(B \operatorname{Spin}(m)) \rightarrow H P \mathbb{Q}^{0}(B \operatorname{Spin}(m))$. Let $\phi \in \bar{K}^{0}(B \operatorname{Spin}(m))$. Then we define

$$
\Phi_{\phi}:=\mathbf{T d}^{-1} \cup \operatorname{ch}(\phi) \in H P \mathbb{Q}^{0}(B \operatorname{Spin}(m)) \cong \mathbb{Q}\left[\left[b^{2} p_{1}, b^{4} p_{2}, \ldots, b^{2 r_{m}} p_{r_{m}}\right]\right]
$$

and obtain $\tilde{\Phi}_{\phi}$ as described above. We define the form

$$
\nu_{u, \phi}:=\operatorname{Td}\left(\tilde{\nabla}^{u}\right)^{-1} \wedge 2 H_{\alpha_{u}} \wedge \tilde{\Phi}_{\phi}\left(\nabla^{m, u}\right) \in \Omega P^{-1}\left(M_{u}\right),
$$

where we use the abbreviation

$$
\tilde{\Phi}_{\phi}\left(\nabla^{m, u}\right):=\tilde{\Phi}_{\phi}\left(p_{1}\left(\nabla^{m, u}\right), p_{2}\left(\nabla^{m, u}\right), \ldots\right) .
$$

We can now define the map

$$
\mathcal{G}_{u}^{\text {String }(m)}: \bar{K}^{0}(B \operatorname{Spin}(m)) \rightarrow \hat{K}^{0}(M)
$$

by the following prescription:

$$
\mathcal{G}_{u}^{\text {String }(m)}(\phi):=\mathcal{G}_{u, 0}^{\text {String }(m)}(\phi)-a\left(\nu_{u, \phi}\right) .
$$

We further define

$$
\mathcal{G}^{\operatorname{String}(m)}:=h^{*} \mathcal{G}_{u}^{\operatorname{String}(m)} .
$$

Unfortunately we can not verify directly that $\mathcal{G}_{u}^{\text {String }(m)}$ is continuous, but we have the following Lemma. 
Lemma 5.16 The map

$$
\mathcal{G}^{\operatorname{String}(m)}: \bar{K}^{0}(B \operatorname{Spin}(m)) \rightarrow \hat{K}^{0}(M)
$$

extends by continuity to $\bar{K}^{0}(B$ String $(m))$. The continuous extension (for which we use the same symbol $\left.\mathcal{G}^{\text {String }(m)}\right)$ admits the degree-preserving cohomological character given by $b^{2 i} p_{i} \mapsto b^{2 i} p_{i}\left(\nabla^{m}\right)$ for all $i \in\left\{2, \ldots, r_{m}\right\}$ and is therefore a geometrisation of $\left(M, f, \tilde{\nabla}^{T M}\right)$.

Proof. Since BString $(2 m)$ has finite skeleta the profinite topology on $\bar{K}^{*}(B \operatorname{String}(2 m))$ has a countable basis of neighbourhoods of zero so that we can check continuity of homomorphisms using sequences converging to zero. Let us consider a sequence $\left(\phi_{k}\right)$ in $\bar{K}^{0}(B \operatorname{Spin}(m))$ such that $p^{*} \phi_{k} \rightarrow 0$ in the profinite topology of $\bar{K}^{0}(B \operatorname{String}(m))$ as $k \rightarrow \infty$. We must show that there exists a $k_{0} \in \mathbb{N}$ such that for all $k \geq k_{0}$ we have $\mathcal{G}^{\text {String }(m)}\left(\phi_{k}\right)=0$. Let $t: N \rightarrow B$ String $(m)$ be a compact $\operatorname{dim}\left(M_{u}\right)+5$-connected approximation. We can choose a $k_{0} \in \mathbb{N}$ such that for all $k \geq k_{0}$ we have $t^{*} p^{*} \phi_{k}=0$ and $t^{*} p^{*}\left(\mathbf{T d}^{-1} \wedge \operatorname{ch}\left(\phi_{k}\right)\right)=0$. Since the pull-back $t^{*}: H^{*}(B \operatorname{String}(m) ; \mathbb{Q}) \rightarrow H^{*}(N ; \mathbb{Q})$ is injective in degrees $\leq \operatorname{dim}\left(M_{u}\right)+4$ we see that $\Phi_{\phi_{k}}$ is a formal power series of terms of homogeneity $\geq \operatorname{dim}\left(M_{u}\right)+5$ (here we count the topological degree, i.e. disregard the degree of $b$ ). Consequently, $i_{p_{1}=0} \Phi_{\phi_{k}}$ is a formal power series of terms of homogeneity $\geq \operatorname{dim}\left(M_{u}\right)+1$.

We now calculate using (104) and (107) that for $\phi \in \bar{K}^{0}(B \operatorname{Spin}(m))$

$$
\begin{aligned}
\operatorname{Td}\left(\tilde{\nabla}^{u}\right) \wedge R\left(\mathcal{G}_{u}^{\text {String }(m)}(\phi)\right) & =\operatorname{Td}\left(\tilde{\nabla}^{u}\right) \wedge R\left(\mathcal{G}_{u}^{\operatorname{Spin}(m)}(\phi)\right)-p_{1}\left(\tilde{\nabla}^{m, u}\right) \wedge \tilde{\Phi}_{\phi}\left(\nabla^{m, u}\right) \\
& =\Phi_{\phi}\left(\nabla^{m, u}\right)-p_{1}\left(\tilde{\nabla}^{m, u}\right) \wedge \tilde{\Phi}_{\phi}\left(\nabla^{m, u}\right) \\
& =\left(i_{p_{1}=0} \Phi_{\phi}\right)\left(\nabla^{m, u}\right) .
\end{aligned}
$$

We conclude that $\mathbf{T d}\left(\tilde{\nabla}^{u}\right) \wedge R\left(\mathcal{G}_{u}^{\text {String }(m)}\left(\phi_{k}\right)\right)=0$ for $k \geq k_{0}$ since $\left(i_{p_{1}=0} \Phi_{\phi}\right)\left(\nabla^{m, u}\right)$ would be a form on $M_{u}$ of a degree which exceeds the dimension. Hence for $k \geq k_{0}$ the class $\mathcal{G}_{u}^{\text {String }(m)}\left(\phi_{k}\right)$ is flat and in the kernel of $I: \hat{K}^{0}\left(M_{u}\right) \rightarrow K^{0}\left(M_{u}\right)$. We conclude that

$$
\mathcal{G}_{u}^{\operatorname{String}(m)}\left(\phi_{k}\right) \in H P \mathbb{R}^{-1}\left(M_{u}\right) / \mathrm{im}(\mathbf{c h}) .
$$

An $\ell+1$-connected map induces an isomorphism in ordinary cohomology in degrees $\leq \ell$. Since BString $(m)$ is rationally even the odd-dimensional real cohomology of the $\ell+1$ connected approximation $M_{u}$ is concentrated in degrees $\geq \ell+1$. Since $\operatorname{dim}(M) \leq \ell$ the restriction $h^{*}: H P \mathbb{R}^{-1}\left(M_{u}\right) \rightarrow H P \mathbb{R}^{-1}(M)$ is trivial. This implies that $\mathcal{G}^{\text {String }(m)}\left(\phi_{k}\right)=$ $h^{*} \mathcal{G}_{u}^{\text {String }(m)}\left(\phi_{k}\right)=0$ for all $k \geq k_{0}$.

The assertion about the cohomological character follows from the relation

$$
\operatorname{Td}\left(\tilde{\nabla}^{T M}\right) \wedge R\left(\mathcal{G}^{\operatorname{String}(m)}(\phi)\right)=\left(i_{p_{1}=0} \Phi_{\phi}\right)\left(\nabla^{m}\right)
$$

derived from (110). This finishes the proof of Lemma 5.16 .

In order to show that this geometrisation $\mathcal{G}^{\text {String(m) }}$ is $\ell$-good we must show that $\mathcal{G}_{u}^{\text {String(m) }}$ is continuous itself. To this end we argue similarly by representing this geometrisation as a pull-back from a $\operatorname{dim}\left(M_{u}\right)+1$-connected approximation of BString $(m)$. 
We now define the geometrization $\mathcal{G}^{\text {String }}$ of $\left[M, f, \tilde{\nabla}^{T M}\right]$ by

$$
\mathcal{G}^{\text {String }}:=\iota_{m, *} \mathcal{G}^{\text {String }(m)}
$$

Since $\iota_{m}$ is $\ell$-connected, this geometrization is $\ell$-good. If $m^{\prime} \in \mathbb{N}$ satisfies $m \leq m^{\prime}$, then it follows from the compatibilty of the family of geometrizations $\left(\mathcal{G}_{u}^{\operatorname{Spin}\left(m^{\prime}\right)}\right)_{m^{\prime} \geq m}$ that

$$
\mathcal{G}^{\operatorname{String}\left(m^{\prime}\right)}(\phi)=\mathcal{G}^{\text {String }(m)}\left(\phi_{\mid B \operatorname{String}(m)}\right), \quad \phi \in \bar{K}^{0}\left(B \operatorname{String}\left(m^{\prime}\right)\right) .
$$

Therefore $\mathcal{G}^{\text {String }}$ does not depend on the choice of $m$. In particular, it is $\ell$-good for all $\ell$. This finishes the proof of Theorem 5.14 .

We now specialise Theorem 4.19 in order to derive an intrinsic formula for

$$
b^{a n}([M, f])=\bar{W} \circ \eta^{a n}([M, f]) \in T_{2 m} .
$$

The connection $\nabla^{k}$ on the $\operatorname{Spin}(k)$-principal bundle $\tilde{f}^{*} Q_{k}^{S p i n} \rightarrow M$ turns the real vector bundle $\tilde{f}^{*} \xi_{k}^{\text {String }}$ into a geometric bundle $\mathbf{N}_{k}$. It is a geometric representative of the stable normal bundle of $M$, hence the notation. We have

$$
R([T M]+1)=R\left(n+1+k-\left[\tilde{f}^{*} \xi_{k}^{\text {String }}\right]\right) \in K[[q]]^{0}(M) .
$$

Therefore we get an interpretation of $\mathbf{R}\left(n+1+k-\mathbf{N}_{k}\right)$ as a virtual geometric representative of $R([T M]+1)$ (which differs from $\mathbf{R}(T M+1)$ used in 101) since we work with the geometry on the normal bundle). By construction we have

$$
\mathcal{G}^{\text {String }}\left(\lambda_{n+1}^{\text {String }}\right)=\left[\mathbf{R}\left(n+1+k-\mathbf{N}_{k}\right)\right]+a\left(\nu_{R\left(\lambda_{n+1}^{\text {Spin }}\right)}\right) \in \hat{K}^{0}(M)[[q]] .
$$

In other words, the correction form for $\left[\lambda_{n+1}^{\text {String }}\right] \in K[[q]]^{0}(B$ String $)$ is given by

$$
\gamma_{R\left(\lambda_{n+1}^{\text {String }}\right)}=\nu_{R\left(\lambda_{n+1}^{\text {Spin }}\right)}=\mathbf{T d}\left(\tilde{\nabla}^{T M}\right)^{-1} \wedge 2 H_{\alpha} \wedge \tilde{\Phi}_{R\left(\lambda_{n+1}^{\text {Spin }}\right)}\left(\nabla^{k}\right) \in \Omega P^{-1}(M)[[q]] .
$$

By Theorem 4.19 the composition $\bar{W} \circ \eta^{t o p}([M, f]) \in T_{2 m}$ is now represented by the formal power series

$$
\left[-\int_{M} 2 H_{\alpha} \wedge \tilde{\Phi}_{R\left(\lambda_{n+1}^{S p i n}\right)}\left(\nabla^{k}\right)\right]-\xi\left(\not D_{M} \otimes \mathbf{R}\left(n+1+k-\mathbf{N}_{k}\right)\right) \in \mathbb{R} / \mathbb{Z}[[q]]
$$

This is the version of (101) using the normal bundle geometry on the twisting bundles.

\subsection{The Crowley-Goette invariants}

In this Subsection we want to show how some of the Eells-Kuiper or Kreck-Stolz type invariants from geometric topology can be understood from the point of view of the universal $\eta$-invariant. Our approach can be described schematically as follows. In a first step, called pre-construction, we translate the geometric topology data into elements of 
a suitable bordism group $M B$. We then apply the universal $\eta$-invariant. Finally, the desired invariant is obtained by a suitable evaluation against classes in $K^{0}(M B)$.

In detail we will consider the example of the Crowley-Goette invariant recently introduced in [24] for $S^{3}$-principal bundles on certain $n=4 m-1$-dimensional manifolds. We start with recalling the definitions from [24]. Since in the present paper we decided to work with Spin $^{c}$-bordism and complex Dirac operators we will define the variant $t_{M}^{\mathbb{C}}$ which coincides with the Crowley-Goette invariant for even $m$ and is its double for odd $m$. Let $S^{3}$ be the group of unit quaternions and $B S^{3}$ be its classifying space. The set of homotopy classes $\left[M, B S^{3}\right]$ is in natural bijection with the set of isomorphism classes of $S^{3}$-principal bundles on $M$ denoted in [24] by $\operatorname{Bun}(M)$.

Let $M$ be a closed $n$-dimensional Spin-manifold such that $H^{3}(M ; \mathbb{Q})=0$ and $H^{4}(M ; \mathbb{Q})=$ 0 . Then the Crowley-Goette invariant is defined as a certain function

$$
t_{M}: \operatorname{Bun}(M) \rightarrow \mathbb{Q} / \mathbb{Z}
$$

In the following we recall the intrinsic formula [24, (1.9)] for $t_{M}$. Note that

$$
H P \mathbb{Q}^{*}\left(B S^{3}\right) \cong \mathbb{Q}\left[b, b^{-1}\right]\left[\left[b^{2} c_{2}\right]\right]
$$

and by the completion theorem [8] we have the isomorphism

$$
K^{0}\left(B S^{3}\right) \cong R\left(S^{3}\right)_{I_{S^{3}}}
$$

We let $\rho$ be the representation of $S^{3}$ by left-multiplication on $\mathbb{H} \cong \mathbb{C}^{2}$. The representation $\rho$ gives rise to a class $[\rho] \in K^{0}\left(B S^{3}\right)$ and a power series $\operatorname{ch}([\rho]) \in \mathbb{Q}\left[b, b^{-1}\right]\left[\left[b^{2} c_{2}\right]\right]^{0}$ of total degree zero. There exists a unique power series $\tilde{\Phi} \in \mathbb{Q}\left[b, b^{-1}\right]\left[\left[b^{2} c_{2}\right]\right]^{-4}$ of total degree -4 such that $2-\operatorname{ch}([\rho])=c_{2} \tilde{\Phi}$.

Let $\tilde{g} \in \operatorname{Bun}(M)$ and $R \rightarrow M$ be a $S^{3}$-bundle classified by $\tilde{g}$. We choose a connection $\nabla^{R}$ on $R$. For every unitary representation $\left(\lambda, V_{\lambda}\right)$ of $S^{3}$ we let $E_{\lambda}:=P \times_{S^{3}, \lambda} V_{\lambda}$ be the vector bundle associated to $R$ and $\lambda$. It comes with a natural hermitean metric $h^{E_{\lambda}}$. The connection $\nabla^{R}$ induces a connection $\nabla^{E_{\lambda}}$ which preserves $h^{E_{\lambda}}$. In this way we get a geometric bundle $\mathbf{E}_{\lambda}:=\left(E_{\lambda}, h^{E_{\lambda}}, \nabla^{E_{\lambda}}\right)$. By our assumptions on the rational cohomology of $M$ the Chern-Weyl representative $c_{2}\left(\nabla^{R}\right)$ of $c_{2}$ is exact, and there exists a unique element $\hat{c}_{2}\left(\nabla^{R}\right) \in \Omega^{3}(M) / \mathrm{im}(d)$ such that $d \hat{c}_{2}\left(\nabla^{R}\right)=c_{2}\left(\nabla^{R}\right)$. We define $\tilde{\Phi}\left(\nabla^{R}\right) \in$ $\Omega P_{c l}^{-4}(M)$ by replacing $c_{2}$ by $c_{2}\left(\nabla^{R}\right)$ in the power series $\tilde{\Phi}$.

We choose a Riemannian metric on $M$ which induces the Levi-Civita connection on $T M$. Furthermore we choose the $S_{p i n}{ }^{c}$-structure induced by the Spin-structure. We then get a natural $\operatorname{Spin}^{c}$-extension $\tilde{\nabla}^{T M}$ of the Levi-Civita connection. The complex version

$$
t_{M}^{\mathbb{C}}: \operatorname{Bun}(M) \rightarrow \mathbb{R} / \mathbb{Z}
$$

of $t_{M}$ is now given by [24, (1.9)]

$$
t_{M}^{\mathbb{C}}(\tilde{g}):=\left[\int_{M} \mathbf{T d}\left(\tilde{\nabla}^{T M}\right) \wedge \hat{c}_{2}\left(\nabla^{R}\right) \wedge \tilde{\Phi}\left(\nabla^{R}\right)\right]-2 \xi\left(\not D_{M}\right)+\xi\left(\not D_{M} \otimes \mathbf{E}_{\rho}\right) \in \mathbb{R} / \mathbb{Z}
$$


To be precise, the value of the integral belongs to $\mathbb{R}\left[b, b^{-1}\right]^{-4}$ which will be identified with $\mathbb{R}$ using the generator $b^{2}$.

In order to relate the Crowley-Goette invariant with the universal $\eta$-invariant we are led to consider a bordism theory of $\operatorname{Spin}^{c}$-manifolds with $S^{3}$-bundles with rationally trivial second Chern class. This bordism theory is constructed homotopy theoretically as follows. We have a fibre sequence

$$
K(\mathbb{Q}, 3) \rightarrow K(\mathbb{Q} / \mathbb{Z}, 3) \stackrel{\partial}{\rightarrow} K(\mathbb{Z}, 4) \rightarrow K(\mathbb{Q}, 4)
$$

of Eilenberg-MacLane spaces. We define the space $X$ by the following homotopy pull-back

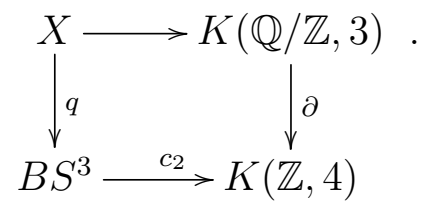

Since $c_{2}$ is a rational isomorphism and $K(\mathbb{Q} / \mathbb{Z}, 3)$ is rationally trivial we see that the space $X$ is rationally contractible.

We consider the Thom spectrum $M B$ associated to the projection

$$
B:=B \operatorname{Spin}^{c} \times X \rightarrow B \operatorname{Spin}^{c} .
$$

We conclude that for $n=4 m-1$

$$
\pi_{n}(M B) \otimes \mathbb{Q} \cong \pi_{n}\left(M \operatorname{Spin}^{c} \wedge X_{+}\right) \otimes \mathbb{Q} \cong \pi_{n}\left(M \operatorname{Spin}^{c} \mathbb{Q} \wedge X_{+}\right) \cong \pi_{n}\left(M \operatorname{Spin}^{c} \mathbb{Q}\right) \cong 0
$$

It follows that

$$
\pi_{n}(M B)_{\text {tors }}=\pi_{n}(M B)
$$

so that the universal $\eta$-invariant is defined on the whole $\operatorname{Spin}^{c}$-bordism group of $X$ :

$$
\eta^{\text {top }}=\eta^{a n}: \pi_{n}(M B) \rightarrow Q_{n}(M B)
$$

The pre-construction for the Crowley-Goette invariant is a map

$$
s_{M}: \operatorname{Bun}(M) \rightarrow \pi_{n}(M B)
$$

given by the following Lemma.

Lemma 5.17 A pair $((M, f), \tilde{g})$ of a cycle $(M, f)$ for $\pi_{n}\left(M \operatorname{Spin}^{c}\right)$ with $H^{3}(M ; \mathbb{Q})=0$ and $H^{4}(M ; \mathbb{Q})=0$, and a map $\tilde{g} \in \operatorname{Bun}(M)$ gives naturally rise to a class $s_{M}(\tilde{g}):=$ $[M,(f, g)] \in \pi_{n}(M B)$.

Proof. The main point is to show that $\tilde{g}: M \rightarrow B S^{3}$ has a natural lift to $g: M \rightarrow X$ in the diagram (112). The rationalisation of $\tilde{g}^{*} c_{2}$ vanishes so that there exists a class $\hat{c}_{2} \in H^{3}(M ; \mathbb{Q} / \mathbb{Z})$ such that $\partial \hat{c}_{2}=\tilde{g}^{*} c_{2}$. This lift is unique up to the image of a rational class of degree 3 , hence unique by our assumption. The map $\tilde{g}$ and the lift 
$\hat{c}_{2}: M \rightarrow K(\mathbb{Q} / \mathbb{Z}, 3)$ together determine the lift $g: M \rightarrow X$.

Our next task is to determine the element in $K^{0}(M B)$ at which want to evaluate. To this end we calculate the $K$-theory $K^{*}(X)$. We have a fibration

$$
X \stackrel{q}{\rightarrow} B S^{3}
$$

with fibre $K(\mathbb{Q}, 3)$. Note that $\mathbb{Q}$ is a countable abelian group. Furthermore, the space $B S^{3}$ has a $C W$-structure with finite skeleta $\left(B S_{k}^{3}\right)_{k \geq 0}$ and $\lim _{k}^{1} K^{*}\left(B S_{k}^{3}\right)=0$ by [8]. We can apply Proposition 6.1 and see that

$$
q^{*}: \bar{K}^{*}\left(B S^{3}\right) \rightarrow \bar{K}^{*}(X)
$$

represents $\bar{K}^{*}(X)$ as a completion of $\bar{K}^{*}\left(B S^{3}\right)$ with respect to the topology induced from the profinite topology on $\bar{K}^{*}(X)$ via $q^{*}$. The domain of this map can be calculated the using the completion theorem [8]. The element $2-\rho$ of the representation $\operatorname{ring} R\left(S^{3}\right)$ generates the dimension ideal $I_{S^{3}}$. If we let $A:=2-[\rho] \in \bar{K}^{0}\left(B S^{3}\right)$, then we have

$$
\bar{K}^{*}\left(B S^{3}\right) \cong \mathbb{Z}[[A]]
$$

Since $X$ is rationally contractible we have

$$
\operatorname{ch}^{*}\left(q^{*} A\right)=q^{*} \operatorname{ch}(A)=0 \text {. }
$$

Let $p: B \operatorname{Spin}^{c} \times X \rightarrow X$ be the projection. By Lemma 2.5 the evaluation

$$
\operatorname{ev}_{p^{*} q^{*} A}: Q_{n}(M B) \rightarrow \mathbb{Q} / \mathbb{Z}
$$

is well-defined. We define

$$
\varepsilon:=\operatorname{ev}_{p^{*} q^{*} A} \circ \eta^{t o p}: \pi_{n}(M B) \rightarrow \mathbb{Q} / \mathbb{Z}
$$

The following proposition clarifies the relation between $t_{M}^{\mathbb{C}}$ and the universal $\eta$-invariant.

Proposition 5.18 If $(M, f)$ is a cycle for $\pi_{n}\left(M \operatorname{Spin}^{c}\right)$ which satisfies $H^{3}(M ; \mathbb{Q})=0$ and $H^{4}(M ; \mathbb{Q})=0$, then we have the relation

$$
t_{M}^{\mathbb{C}}=\varepsilon \circ s_{M}: \operatorname{Bun}(M) \rightarrow \mathbb{Q} / \mathbb{Z}
$$

Proof. It is an instructive exercise in the use of geometrisations to derive an intrinsic formula for the composition $\varepsilon \circ s_{M}$ which can be compared with the formula 111 for $t_{M}^{\mathbb{C}}$. In a first step we must approximate the space $X$ by spaces with finite skeleta. Note that we can write (compare with (35) for the connecting maps)

$$
K(\mathbb{Q} / \mathbb{Z}, 3):=\operatorname{hocolim}_{l} K(\mathbb{Z} / l \mathbb{Z}, 3)
$$


If we define $X_{l}$ by the pull-back

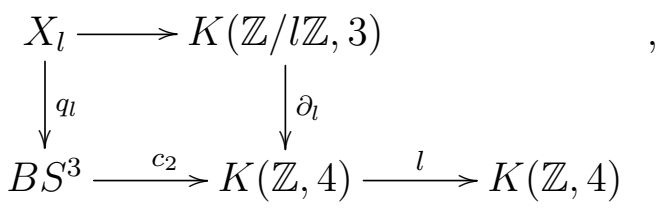

then we get connecting maps $X_{l} \rightarrow X_{l^{\prime}}$ if $l \mid l^{\prime}$ and

$$
X \cong \operatorname{hocolim}_{l} X_{l}, \quad \pi_{n}(M B)=\operatorname{colim} \pi_{n}\left(M B_{l}\right)
$$

where the Thom spectrum $M B_{l}$ is associated to the projection $B_{l}:=B \operatorname{Spin}^{c} \times X_{l} \rightarrow$ $B$ Spin $^{c}$. The main advantage of $X_{l}$ is that it has finite skeleta.

We consider a cycle $(M, f)$ for $\pi_{n}\left(M \operatorname{Spin}^{c}\right)$ and an auxiliary map $g: M \rightarrow X$. We can assume that $g$ has a factorisation

$$
g: M \stackrel{g_{l}}{\rightarrow} X_{l} \rightarrow X
$$

for some $l$. We choose a Spin ${ }^{c}$-extension $\tilde{\nabla}^{T M}$ of the Levi-Civita connection. We are going to construct an $\ell$-good geometrisation for $\left(M,\left(f, g_{l}\right), \tilde{\nabla}^{T M}\right)$ using similar ideas as in the String-bordism case Proposition 5.14. We first take $m$ sufficiently large such that the canonical map $\iota_{m}: B \operatorname{Spin}^{c}(m) \rightarrow B \operatorname{Spin}^{c}$ is $\max \{\ell, n+1,4\}$-connected. We choose a compact $\max (\ell, n+1,4)$-connected approximation $\left(f_{u}, g_{u}\right): M_{u} \rightarrow B \operatorname{Spin}^{c}(m) \times X_{l}$ such that the map $\left(f, g_{l}\right)$ factorizes over a closed embedding $h: M \rightarrow M_{u}$. It is here where we use the property that $X_{l}$ has finite skeleta which ensures that we can find a compact approximation $M_{u}$.

We choose the $\operatorname{Spin}^{c}(m)$-connection $\tilde{\nabla}^{u}$ as in Subsection 4.4. The map $h$ has a refinement to a $\operatorname{Spin}^{c}$-map and we can assume that $h^{*} \tilde{\nabla}^{u}=\tilde{\nabla}^{T M}$ stably.

The composition $q_{l} \circ g_{u}: M_{u} \rightarrow B S^{3}$ classifies an $S^{3}$-principal bundle $R_{u} \rightarrow M_{u}$ on which we choose a connection $\nabla^{R_{u}}$. We can assume that $R \cong h^{*} R_{u}$ with connection $\nabla^{R}=h^{*} \nabla^{R_{u}}$.

We let

$$
\tilde{\mathcal{G}}_{u}: K^{0}\left(B \operatorname{Spin}^{c}(m) \times B S^{3}\right) \rightarrow \hat{K}^{0}\left(M_{u}\right)
$$

denote the geometrisation of $\left(M_{u},\left(f_{u}, q_{l} \circ g\right), \tilde{\nabla}^{u}\right)$ which was constructed by the method of Lemma 4.7 from a geometrization $\mathcal{G}^{0}:=\mathcal{G}^{\operatorname{Spin}^{c}(m)}$ which is the analog of $\mathcal{G}^{\operatorname{Spin}(m)}$ in the proof of Proposition 5.13 .

We have a fibration

$$
\operatorname{BSpin}^{c}(m) \times X_{l} \stackrel{\left(\mathrm{id}, q_{l}\right)}{\rightarrow} \operatorname{BSpin}^{c}(m) \times B S^{3}
$$

with fibre $K(\mathbb{Z}, 3)$. We can again apply Proposition 6.1 and conclude that

$$
\left(\mathrm{id}, q_{l}\right)^{*}: \bar{K}^{*}\left(\operatorname{BSpin}^{c}(m) \times B S^{3}\right) \rightarrow \bar{K}^{*}\left(B \operatorname{Spin}^{c}(m) \times X_{l}\right)
$$

represents $\bar{K}^{*}\left(B \operatorname{Spin}^{c}(m) \times X_{l}\right)$ as a completion of $\bar{K}^{*}\left(\operatorname{BSpin}^{c}(m) \times B S^{3}\right)$. We define

$$
\mathcal{G}_{u, 0}: \bar{K}^{*}\left(B \operatorname{Spin}^{c}(m) \times B S^{3}\right) \rightarrow \hat{K}^{0}\left(M_{u}\right)
$$


by

$$
\mathcal{G}_{u, 0}(\phi):=\tilde{\mathcal{G}}_{u}(\phi) \in \hat{K}^{0}\left(M_{u}\right), \quad \phi \in \bar{K}^{*}\left(B \operatorname{Spin}^{c}(m) \times B S^{3}\right) .
$$

This map needs a correction in order to be continuous with respect to the topology on $\bar{K}^{*}\left(\operatorname{BSpin}^{c}(m) \times B S^{3}\right)$ induced from the profinite topology of $\bar{K}^{*}\left(\operatorname{BSpin}^{c}(m) \times X_{l}\right)$. We must kill the contribution of $c_{2}\left(\nabla^{R_{u}}\right)$ to the curvature of $\mathcal{G}_{u, 0}(\phi)$. Note that $q_{l}^{*} c_{2} \in$ $H^{4}\left(X_{l} ; \mathbb{Z}\right)$ is $l$-torsion. Hence we can choose a form $\alpha_{u} \in \Omega^{3}\left(M_{u}\right) / \operatorname{im}(d)$ such that $d \alpha_{u}=$ $c_{2}\left(\nabla^{R_{u}}\right)$. By an easy application of Serre's spectral sequence to the fibration 115 we see that

$$
p^{*}: H^{*}\left(\operatorname{BSpin}^{c}(m) ; \mathbb{Q}\right) \rightarrow H^{*}\left(\operatorname{BSpin}^{c}(m) \times X_{l} ; \mathbb{Q}\right)
$$

is an isomorphism. Since $H^{*}\left(B \operatorname{Spin}^{c}(m) ; \mathbb{Q}\right)$ is concentrated in even degrees the odddimensional cohomology of $M_{u}$ is concentrated in degrees $\geq n+1$. In particular we see that $\alpha_{u}$ is uniquely determined. Moreover, the restriction

$$
h^{*}: H P \mathbb{R}^{-1}\left(M_{u}\right) \rightarrow H P \mathbb{R}^{-1}(M)
$$

is trivial.

We have

$$
\begin{aligned}
H P \mathbb{Q}^{*}\left(\operatorname{SSpin}^{c}(m) \times B S^{3}\right) & \cong \mathbb{Q}\left[b, b^{-1}\right]\left[\left[b c_{1}, b^{2} p_{1}, b^{4} p_{2} \ldots, b^{2 r_{m}} p_{r_{m}}, b^{2} c_{2}\right]\right], \\
H P \mathbb{Q}^{*}\left(\operatorname{Sipin}^{c}(m) \times X_{l}\right) & \cong \mathbb{Q}\left[b, b^{-1}\right]\left[\left[b c_{1}, b^{2} p_{1}, b^{4} p_{2} \ldots, b^{2 r_{m}} p_{r_{m}}\right]\right],
\end{aligned}
$$

where $c_{1}$ and the Pontrjagin classes come from $B \operatorname{Spin}^{c}$, and $c_{2}$ is pulled back from $B S^{3}$. The pull-back (id, $\left.q_{l}\right)^{*}$ is the quotient map defined by setting $c_{2}=0$. For $\phi \in$ $K^{0}\left(\operatorname{BSpin}^{c}(m) \times B S^{3}\right)$ we define the formal power series

$$
\Phi_{\phi}:=\mathbf{T d}^{-1} \cup \mathbf{c h}(\phi) \in \mathbb{Q}\left[b, b^{-1}\right]\left[\left[b c_{1}, b^{2} p_{1}, b^{4} p_{2} \ldots, b^{2} c_{2}\right]\right]^{0}
$$

and set

$$
\tilde{\Phi}_{\phi}:=\frac{\Phi_{\phi}-i_{c_{2}=0} \Phi_{\phi}}{c_{2}} \in \mathbb{Q}\left[b, b^{-1}\right]\left[\left[b c_{1}, b^{2} p_{1}, b^{4} p_{2} \ldots, b^{2} c_{2}\right]\right]^{-4} .
$$

For $\phi \in \bar{K}^{0}\left(B \operatorname{Spin}^{c}(m) \times B S^{3}\right)$ now define

$$
\mathcal{G}_{u}(\phi):=\mathcal{G}_{u, 0}(\phi)-a\left(\alpha_{u} \wedge \mathbf{T d}\left(\tilde{\nabla}^{u}\right)^{-1} \wedge \tilde{\Phi}_{\phi}\left(\nabla^{u}, \nabla^{R_{u}}\right)\right),
$$

where $\tilde{\Phi}_{\phi}\left(\nabla^{u}, \nabla^{R_{u}}\right) \in \Omega P^{-4}(M)$ is obtained from $\tilde{\Phi}_{\phi}$ by replacing the generators $c_{1}, p_{i}$ and $c_{2}$ by their corresponding Chern-Weyl representatives $c_{1}\left(\tilde{\nabla}^{u}\right), p_{i}\left(\tilde{\nabla}^{u}\right)$, and $c_{2}\left(\nabla^{R_{u}}\right)$. We calculate similarly as in 110 that

$$
\operatorname{Td}\left(\tilde{\nabla}^{u}\right) \wedge R\left(\mathcal{G}_{u}(\phi)\right)=i_{c_{2}=0} \Phi_{\phi}\left(\nabla^{u}, \nabla^{R_{u}}\right) .
$$

We now define

$$
\mathcal{G}: \bar{K}^{*}\left(B \operatorname{Spin}^{c} \times B S^{3}\right) \rightarrow \hat{K}^{0}(M)
$$

by

$$
\mathcal{G}(\phi):=\left(\iota_{m} \times \operatorname{id}_{X_{l}}\right)_{*} h^{*} \mathcal{G}_{u}(\phi)
$$


We claim that $\mathcal{G}$ extends by continuity to a good geometrisation of $\left(M,\left(f, g_{l}\right), \tilde{\nabla}^{T M}\right)$. The argument is very similar to that of Lemma 5.16. We first show continuity. If $\left(\phi_{k}\right)$ is a sequence in $\bar{K}^{0}\left(B \operatorname{Spin}^{c} \times B S^{3}\right)$ with $\left(\mathrm{id}, q_{l}\right)^{*} \phi_{k} \rightarrow 0$ as $k \rightarrow \infty$ in the profinite topology, then we can find a $k_{0} \in \mathbb{N}$ such that

$$
\mathcal{G}_{u}\left(\phi_{k}\right) \in H P \mathbb{R}^{-1}\left(M_{u}\right) / \operatorname{im}(\mathbf{c h})
$$

for all $k \geq k_{0}$. It follows from the vanishing of the map $h^{*}$ in $(117)$ that $h^{*} \mathcal{G}_{u}\left(\phi_{k}\right)=0$. Because of (118) a degree-preserving cohomological character of $\mathcal{G}$ is given by

$$
b c_{1} \mapsto b c_{1}\left(\tilde{\nabla}^{T M}\right), \quad b^{2 i} p_{i} \mapsto b^{2 i} p_{i}\left(\tilde{\nabla}^{T M}\right) .
$$

It follows that $\mathcal{G}$ is a geometrisation. In order to see that it is $\ell$-good we show that $\mathcal{G}_{u}$ itself is continuous using a similar argument based on a $\operatorname{dim}\left(M_{u}\right)+1$-connected approximation of $B \operatorname{Spin}^{c}(m) \times X_{l}$.

We can now apply Theorem 4.19 in order to derive a formula for $\varepsilon\left(\left[M,\left(f, g_{l}\right)\right]\right) \in \mathbb{R} / \mathbb{Z}$. We can take $\hat{c}_{2}\left(\nabla^{R}\right):=h^{*} \alpha_{u}$ and have $\tilde{\Phi}_{A}=\mathbf{T d}^{-1} \tilde{\Phi}$. We have by construction

$$
\mathcal{G}\left(\left(\mathrm{id}, q_{l}\right)^{*} A\right)=\left[2-\mathbf{E}_{\rho}\right]-a\left(\hat{c}_{2}\left(\nabla^{R}\right) \wedge \tilde{\Phi}\left(\nabla^{R}\right)\right),
$$

hence the correction form (Definition 4.18) is given by

$$
\gamma_{\left(\mathrm{id}, q_{l}\right)^{*} A}=-\hat{c}_{2} \wedge \tilde{\Phi}\left(\nabla^{R}\right)
$$

It follows from $(68)$ that

$\mathrm{ev}_{\left(\mathrm{id}, q_{l}\right)^{*} A}\left(\eta^{a n}\left(\left[M,\left(f, g_{l}\right)\right]\right)\right)=\left[\int_{M} \mathbf{T d}\left(\tilde{\nabla}^{T M}\right) \wedge \hat{c}_{2}\left(\nabla^{R}\right) \wedge \tilde{\Phi}\left(\nabla^{R}\right)\right]-2 \xi\left(\not D_{M}\right)+\xi\left(\not D_{M} \otimes \mathbf{E}\right)$

This is exactly the formula (111) for $t_{M}^{\mathbb{C}}(\tilde{g}) \in \mathbb{R} / \mathbb{Z}$. The Proposition 5.18 now follows from Lemma 2.4 which gives the first equality in the chain

$$
\mathrm{ev}_{\left(\mathrm{id}, q_{l}\right)^{*} A}\left(\eta^{a n}\left(\left[M,\left(f, g_{l}\right)\right]\right)\right)=\mathrm{ev}_{(\mathrm{id}, q)^{*} A}\left(\eta^{a n}([M,(f, g)])\right)=\varepsilon\left(s_{M}(\tilde{g})\right) \text {. }
$$

The paper 24] provides a lot of interesting explicit calculations. Our general point of view is probably not of much help here. But it is useful to understand structural results like the relation with the Adams $e$-invariant [24, Prop 1.11]. This is what we are going to explain now. We define the space $Y$ by extending the diagram $(112)$ by another cartesian square as follows

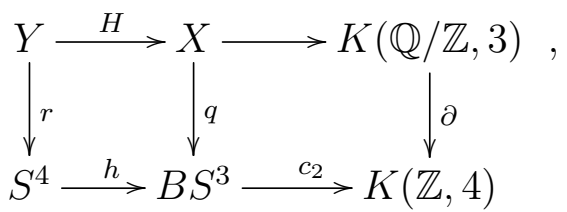


where $h$ generates $\pi_{4}\left(B S^{3}\right)$ such that $h^{*} c_{2} \in H^{4}\left(S^{4} ; \mathbb{Z}\right)$ is the positive orientation class. We use the Serre spectral sequence in order to calculate the rational cohomology of $Y$ :

$$
H^{k}(Y ; \mathbb{Q})=\left\{\begin{array}{cc}
\mathbb{Q} & k=0,7 \\
0 & k \notin\{0,7\}
\end{array} .\right.
$$

This implies

$$
\pi_{4 m-1}\left(S \wedge Y_{+}\right)_{\text {tors }}=\pi_{4 m-1}\left(S \wedge Y_{+}\right)
$$

for $m \geq 3$.

From now on we assume that $m \geq 2$. By Lemma 2.4 we get the commutativity of the squares (except of the lower right which will be explained below) of the following diagram:

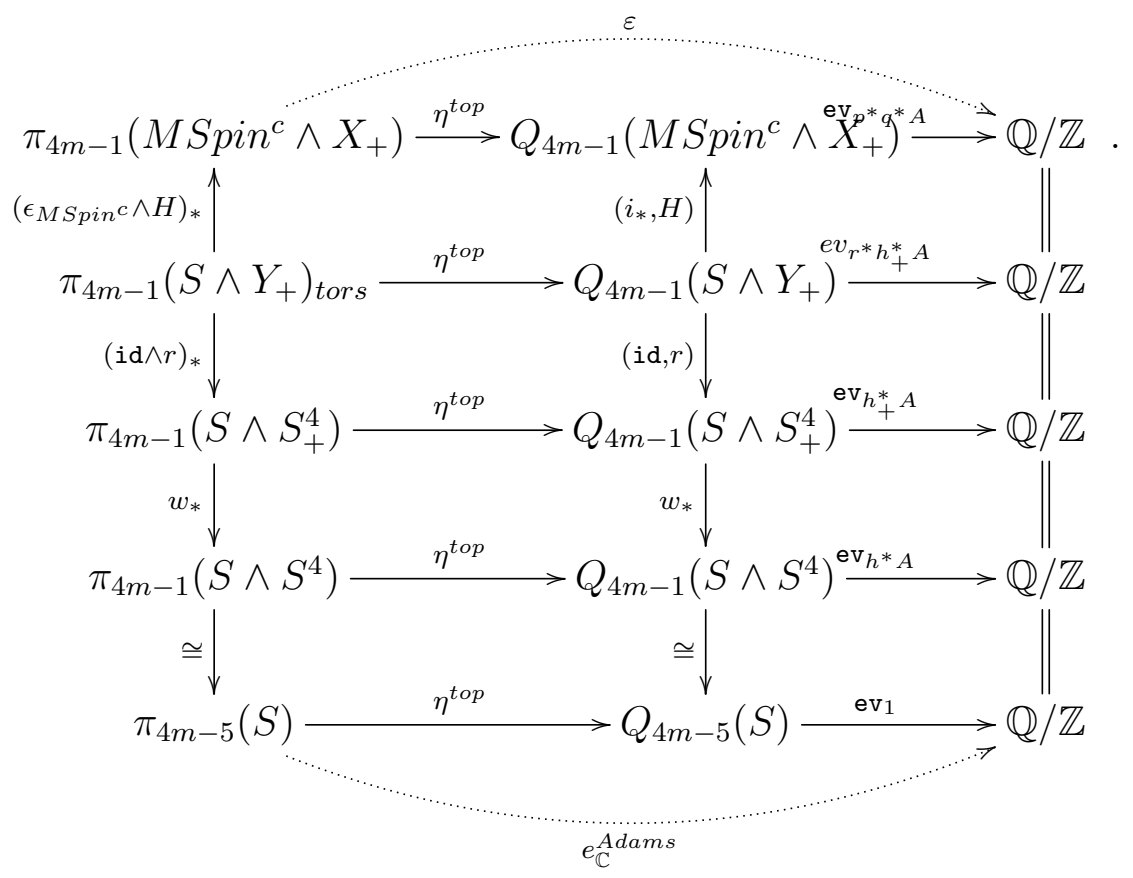

We need the condition $m \geq 2$ in order to have well-defined evaluations $\operatorname{ev}_{h_{+}^{*} A}$, $\operatorname{ev}_{h^{*} A}$ and $\mathrm{ev}_{1}$. The map $w_{*}$ is induced by the map $w: S_{+}^{4} \rightarrow S^{4}$ which is the identity on $S^{4}$ and maps the extra base point to the base point of $S^{4}$. In terms of the canonical decomposition

$$
K^{0}\left(S_{+}^{4}\right) \cong K^{0}\left(S^{4}\right) \oplus \mathbb{Z}
$$

we have $w^{*}=\left(\operatorname{id}_{K^{0}\left(S^{4}\right)}\right.$, dim). This map induces

$$
w_{*}: Q_{4 m-1}\left(S \wedge S_{+}^{4}\right) \rightarrow Q_{4 m-1}\left(S \wedge S^{4}\right)
$$

We use the symbol $h_{+}: S_{+}^{4} \rightarrow B S^{3}$ for the map induced by $h$ which maps the extra base point to a base point of $B S^{3}$. The lower left vertical map is the suspension isomorphism. The lower middle vertical isomorphism is again induced by suspension and the Bott isomorphism

$$
K^{0}\left(S^{4}\right) \cong K^{-4}\left(S^{0}\right) \stackrel{b^{-2}}{\rightarrow} K^{0}\left(S^{0}\right)
$$


In order to see that the lower right square commutes note that this isomorphism maps $h^{*} A$ to 1 . This follows from

$$
\operatorname{ch}(2-A)=b c_{2}+O\left(b^{3}\right)
$$

and the fact that $c_{2} \in H^{4}\left(S^{4} ; \mathbb{Z}\right)$ is the suspension of $1 \in H^{0}(* ; \mathbb{Z})$. The composition of the lower two arrows is the definition (69) of the complex version of the Adams $e$-invariant. We conclude that

$$
\varepsilon \circ\left(\epsilon_{M \operatorname{Spin}^{c}} \wedge H\right)=e_{\mathbb{C}}^{\text {Adams }} \circ w_{*} .
$$

The same argument as for Lemma 5.17 gives

Lemma 5.19 A pair $((M, f), \tilde{g})$ of a cycle for $\pi_{4 m-1}(S)$ such that $H^{3}(M ; \mathbb{Q})=0$ and $H^{4}(M ; \mathbb{Q})=0$, and a map $\tilde{g} \in\left[M, S^{4}\right]$ gives naturally rise to a class $[M,(f, g)] \in$ $\pi_{4 m-1}\left(S \wedge Y_{+}\right)$.

If $M$ satisfies the assumption of the Lemma, then we have a preconstruction map

$$
\tilde{s}_{M}:\left[M, S^{4}\right] \rightarrow \pi_{4 m-1}\left(S \wedge Y_{+}\right)
$$

and conclude from Proposition 5.18, 122) and (120) that for $m \geq 3$ (or $m=2$ and $[M,(f, g)] \in \pi_{7}\left(S \wedge Y_{+}\right)$is a torsion class $)$

$$
t_{M}^{\mathbb{C}}=e_{\mathbb{C}}^{\text {Adams }} \circ w_{*} \circ \tilde{s}_{M}:\left[M, S^{4}\right] \rightarrow \mathbb{Q} / \mathbb{Z} .
$$

This is [24, Prop 1.11] if one takes the following geometric description of the composition $w_{*} \circ \tilde{s}_{M}(\tilde{g})$ into account. First of all we have $\tilde{s}_{M}(\tilde{g})=[M,(f, g)]$, where $g: M \rightarrow Y$ is the lift of $\tilde{g}: M \rightarrow S^{4}$. Then $w_{*}([M,(f, g)])=[M,(f, \tilde{g})]-[M$, const $] \in \pi_{4 m-1}\left(S \wedge S^{4}\right)$. The geometric representative of the 4-fold desuspension of this class is the stably normally framed manifold obtained by taking the preimage $Y:=\tilde{g}^{-1}(s)$ of a regular point $s \in S^{4}$ of $\tilde{g}$.

Corollary 5.20 24, Prop 1.11 We assume that $m \geq 2$. Let $(M,(f, \tilde{g}))$ be as in Lemma 5.19. If $m=2$, then in addition we assume that $[M,(f, g)] \in \pi_{7}\left(S \wedge Y_{+}\right)$is a torsion class. Then we have

$$
t_{M}^{\mathbb{C}}(h \circ \tilde{g})=e_{\mathbb{C}}^{A d a m s}\left(\left[Y, f^{\prime}\right]\right),
$$

where $Y$ is the preimage $Y:=\tilde{g}^{-1}(s)$ of a regular point $s \in S^{4}$ of $\tilde{g}$ with its induced normal framing (and $f^{\prime}$ is the constant map).

Remark 5.21 In the following we consider the case $m=2$ and discuss what happens if we drop the assumption that $[M,(f, g)] \in \pi_{4 m-1}\left(S \wedge Y_{+}\right)$is a torsion element. We consider the Hopf fibration $\tilde{g}: S^{7} \rightarrow S^{4}$. By Lemma 5.19 we get an element $\left[S^{7},(f, g)\right] \in \pi_{7}\left(S \wedge Y_{+}\right)$. This element is not torsion.

\footnotetext{
${ }^{1}$ The $e$-invariant used in the present paper is the negative of the $e$-invariant in the conventions of [24, Prop 1.11]. This accounts for the different sign.
} 
If it would be a torsion element, then by Corollary 5.20 we would have $t_{S^{7}}^{\mathbb{C}}(h \circ \tilde{g})=$ $e_{\mathbb{C}}^{\text {Adams }}\left(\left[Y, f^{\prime}\right]\right)$, where $\left(Y, f^{\prime}\right)$ is a Hopf fibre with the induced framing. It has been shown in [24, Example 3.5] that $t_{S^{7}}^{\mathbb{C}}(h \circ \tilde{g})=0$. On the other hand, since the Hopf fibration generates the stable homotopy group $\pi_{3}(S) \cong \mathbb{Z} / 24 \mathbb{Z}$ which is detected completely by $e^{\text {Adams }}$ we know that $e_{\mathbb{C}}^{\text {Adams }}\left(\left[Y, f^{\prime}\right]\right) \in \mathbb{Q} / \mathbb{Z}$ has order 12 , in particular is non-trivial.

Now

$$
\mathrm{ev}_{h_{+}^{*} A}\left(\eta^{\text {top }}\left((\mathrm{id} \wedge r)_{*}\left[S^{7},(f, g)\right]\right)\right)=e_{\mathbb{C}}^{\text {Adams }}\left(\left[Y, f^{\prime}\right]\right) \neq 0
$$

is a non-trivial torsion class of order 12 . On the other hand

$$
\mathrm{ev}_{p^{*} q^{*} A}\left(\eta^{t o p}\left(\left(\epsilon_{M \operatorname{Spin}^{c}} \wedge H\right)_{*}\left[S^{7},(f, g)\right]\right)\right)=t_{S^{7}}^{\mathbb{C}}(h \circ \tilde{g})=0 .
$$

We see that the upper half of the diagram (121) no longer commutes if we delete the subscript $(\ldots)_{\text {tors }}$ in the second line.

\section{The $K$-theory of $K(\pi, n)$-bundles}

The goal of this Section is to give a proof of Proposition 5.15. It uses some theory which we develop in greater generality for the purpose of applications at other places. The main result is Proposition 6.1.

Let $Z$ be a space with an increasing filtration

$$
\cdots \subseteq Z_{k} \subseteq Z_{k+1} \subseteq \ldots, \quad k \in \mathbb{N}
$$

such that $Z \simeq$ hocolim $\operatorname{siN}_{k} Z_{k}$. Then we consider the decreasing filtration

$$
F^{k} K^{*}(Z):=\operatorname{ker}\left(K^{*}(Z) \rightarrow K^{*}\left(Z_{k-1}\right)\right)
$$

of the $K$-theory group $K^{*}(Z)$, and we write $\operatorname{Gr}_{F}^{k}\left(K^{*}(Z)\right)$ for its associated subquotients. The filtration $\left(F^{k} K^{*}(Z)\right)_{k \in \mathbb{N}}$ induces a topology on $K^{*}(Z)$, and we write ${ }^{F} \bar{K}^{*}(Z)$ for the Hausdorff completion of $K^{*}(Z)$ with respect to this topology.

If $Z^{\prime}$ is a second space with increasing filtration $\left(Z_{k}^{\prime}\right)_{k \in \mathbb{N}}$ and $Z \rightarrow Z^{\prime}$ is a filtration preserving map, then the pull-back $K^{*}\left(Z^{\prime}\right) \rightarrow K^{*}(Z)$ is fitration preserving, continuous and induces a continuous map ${ }^{F} \bar{K}^{*}\left(Z^{\prime}\right) \rightarrow{ }^{F} \bar{K}^{*}(Z)$.

If the $Z_{k}, k \in \mathbb{N}$, are finite $C W$-complexes, then the induced topology on $K^{*}(Z)$ is the profinite topology. For a general filtration of $Z$ the profinite topology is always contained in the topology associated to the filtration and we have a continuous map ${ }^{F} \bar{K}^{*}(Z) \rightarrow \bar{K}^{*}(Z)$.

The filtration of $Z$ induces a spectral sequence $\left(E_{r}, d_{r}\right)$ with $E_{1}^{s, t} \cong K^{t+s}\left(Z_{s} / Z_{s-1}\right)$. If the filtration of $Z$ is the skeletal filtration, then this is the Atiyah-Hirzebruch spectral sequence whose second page is given by $E_{2}^{s, t} \cong H^{s}\left(Z, \pi_{-t}(K)\right)$.

If we fix $(s, t) \in \mathbb{N} \times \mathbb{Z}$, then for $r \geq s$ the group $E_{r}^{s, t}$ does not receive differentials. Hence we can consinder the sequence of inclusions of groups

$$
E_{\infty}^{s, t}:=\bigcap_{r \geq s} E_{r}^{s, t} \subseteq \cdots \subseteq E_{r+2}^{s, t} \subseteq E_{r+1}^{s, t} \subseteq E_{r}^{s, t}
$$


For every $k \in \mathbb{N}$ there is a natural map

$$
\sigma_{k}: \operatorname{Gr}_{F}^{k} K^{t+k}(Z) \rightarrow E_{\infty}^{k, t} .
$$

We say that the spectral sequence converges strongly if $(123)$ is an isomorphism for all $k \in \mathbb{N}$ and $t \in \mathbb{Z}$. This is the case for example if the spectral sequence degenerates at a finite stage.

Proposition 6.1 Let $X$ be a $C W$-complex with an increasing filtration $\left(X_{k}\right)_{k \in \mathbb{N}}$ by finite subcomplexes $X_{k}$ such that $X=\bigcup_{k} X_{k}$. We further fix an integer $n \geq 3$, a countable abelian group $\pi$, and consider a fibration $p: Y \rightarrow X$ with fibre $K(\pi, n)$. Let $\left(Y_{k}\right)_{k \in \mathbb{N}}$ be the induced filtration of $Y$.

1. The projection $p: Y \rightarrow X$ induces a continuous injective map

$$
\bar{K}^{*}(X) \rightarrow{ }^{F} \bar{K}^{*}(Y) .
$$

2. If $\lim _{k}^{1} K^{*}\left(X_{k}\right)=0$, then the image of the composition

$$
\bar{K}^{*}(X) \rightarrow{ }^{F} \bar{K}^{*}(Y) \rightarrow \bar{K}^{*}(Y)
$$

is dense.

Remark 6.2 If $\pi$ is a countable torsion group, then [4, Prop. 4.7] gives the stronger statement that $p^{*}: K^{*}(X) \rightarrow K^{*}(Y)$ is an isomorphism. In this case we can even assume that $n \geq 2$, and we can drop the assumption that $X$ has finite skeleta.

Proof. [of Proposition 5.15 assuming Proposition 6.1 Proposition 5.15 follows from the combination of the two assertions of Proposition 6.1 if we take $n:=3, \pi:=\mathbb{Z}$, and $p: B \operatorname{String}(m) \rightarrow B \operatorname{Spin}(m)$ (note that $m \geq 3$ ). The homotopy type $B \operatorname{Spin}(m)$ admits a cell structure with finite skeleta. Moreover, it has been shown in [8, Sec. 2] that $\lim _{k}^{1} K^{*}\left(B \operatorname{Spin}(m)_{k}\right)=0$.

In order to show Proposition 6.1 we first need some preparations about divisible groups. Let $A$ be some abelian group. Then we define its subgroup

$$
A_{d i v}:=\left\{a \in A \mid \forall n \in \mathbb{N} \exists a^{\prime} \in A \text { s.t. } a=n a^{\prime}\right\}
$$

of divisible elements. We consider the exact sequence

$$
0 \rightarrow A_{\text {div }} \rightarrow A \rightarrow \bar{A} \rightarrow 0 .
$$

Since a divisible group is injective this sequence is split. Hence we have a non-canonical decomposition

$$
A \cong A_{d i v} \oplus \bar{A} .
$$

This implies that $\bar{A}_{d i v}=0$. We now consider a short exact sequence of groups

$$
0 \rightarrow A \rightarrow B \rightarrow C \rightarrow 0
$$

together with a homomorphism $B \rightarrow X$, where $X$ is a finitely generated abelian group. 
Lemma 6.3 If $c \in C_{d i v}$, then we can find a lift $b \in B$ of $c$ whose image in $X$ vanishes. Proof. We consider the diagram

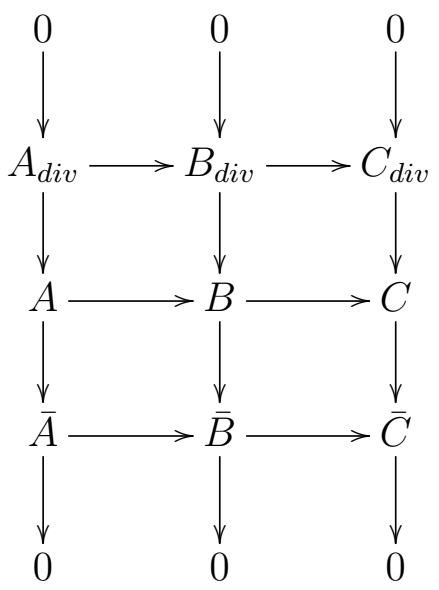

with vertical exact sequences. The Snake Lemma gives an isomorphism

$$
\frac{C_{d i v}}{\operatorname{im}\left(B_{d i v} \rightarrow C_{d i v}\right)} \cong \frac{\operatorname{ker}(\bar{B} \rightarrow \bar{C})}{\operatorname{im}(\bar{A} \rightarrow \bar{B})} .
$$

This shows that the group on the right-hand side is divisible. Since any map from a divisible group to a finitely generated group is trivial we have a factorisation $\bar{B} \rightarrow X$ of the map $B \rightarrow X$. We thus get a homomorphism

$$
\frac{\operatorname{ker}(\bar{B} \rightarrow \bar{C})}{\operatorname{im}(\bar{A} \rightarrow \bar{B})} \rightarrow \frac{X}{Y},
$$

where $Y \subseteq X$ is the image of $\bar{A} \rightarrow \bar{B} \rightarrow X$. The quotient $X / Y$ is still finitely generated, and this implies that this map is trivial since its domain is divisible.

We now choose a preimage $b_{0} \in B$ of $c$. Its image in $\bar{b}_{0} \in \bar{B}$ then vanishes when mapped to $\bar{C}$ so that it represents a class in $\frac{\operatorname{ker}(\bar{B} \rightarrow \bar{C})}{\operatorname{im}(\bar{A} \rightarrow \bar{B})}$. The image of this class in $X / Y$ vanishes so that there exists $\bar{a} \in \bar{A}$ such that the image of $\bar{b}_{0}-\bar{a}$ in $X$ vanishes. We choose some lift $a \in A$ of $\bar{a}$. Then the image of $b:=b_{0}-a$ in $X$ vanishes. Moreover, $b$ can be taken as a lift of $c$, too.

Proposition 6.4 Assume that $X$ is a finite $C W$-complex and $p: Y \rightarrow X$ is a fibration with fibre $K(\pi, n)$ for $n \geq 3$ and $\pi$ a countable abelian group. Then the map

$$
p^{*}: K(X) \rightarrow K^{*}(Y)
$$

is injective and has dense range. 
Proof. Let

$$
\emptyset=X_{-1} \subseteq X_{0} \subseteq X_{1} \subseteq \cdots \subseteq X_{\operatorname{dim}(X)}=X
$$

be the filtration of $X$ given by the cellular structure. It induces a filtration

$$
\emptyset=Y_{-1} \subseteq Y_{0} \subseteq Y_{1} \subseteq \cdots \subseteq Y_{\operatorname{dim}(X)}=Y
$$

by taking preimages. These filtrations induce spectral sequences $E($ id $)$ and $E(p)$ which both degenerate at the $\operatorname{dim}(X)$ 'th page and strongly converge. Here $E(i d)$ is the AtiyahHirzebruch spectral sequence for $X$, and $E(p)$ is the Serre spectral sequence for the fibration $p$.

We consider the map of fibrations

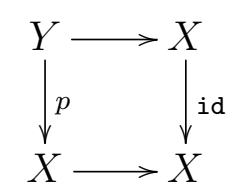

which induces a morphism of spectral sequences

$$
p^{*}: E(\mathrm{id}) \rightarrow E(p) .
$$

Lemma 6.5 The image $p^{*} E(\mathrm{id})$ is a direct summand of $E(p)$.

Proof. We have

$$
E_{1}^{s, t}(p) \cong C^{s}\left(X, K^{t-s}(K(\pi, n))\right),
$$

where $C^{s}(X, A)$ denotes the cellular cochains of $X$ with coefficients in the abelian group $A$. We have an exact sequence

$$
0 \rightarrow E_{1}^{s, t}(p)_{d i v} \rightarrow E_{1}^{s, t}(p) \rightarrow \bar{E}_{1}^{s, t}(p) \rightarrow 0
$$

Since $\tilde{K}^{*}(K(\pi, n))$ is uniquely divisible by [4, Thm. II] the composition

$$
E_{1}^{s, t}(\mathrm{id}) \stackrel{p^{*}}{\rightarrow} E_{1}^{s, t}(p) \rightarrow \bar{E}_{1}^{s, t}(p)
$$

is an isomorphism. Moreover, since $p^{*}$ is a chain map and there are no non-trivial maps from a divisible group to a finitely generated group we have a decomposition of chain complexes

$$
E_{1}^{*, *}(p) \cong E_{1}^{*, *}(p)_{d i v} \oplus p^{*} E_{1}^{*, *}(\mathrm{id}) .
$$

Using that $E_{1}^{*, *}(p)_{d i v}$ is actually uniquely divisible and that a subquotient of a uniquely divisible group is again uniquely divisible we obtain an induced decomposition of the higher pages and a decomposition of the whole spectral sequence as

$$
E(p) \cong E(p)_{\operatorname{div}} \oplus p^{*} E(\mathrm{id})
$$


Lemma 6.6 The map 126 is injective.

Proof. Assume that $\phi \in K^{*}(X)$ is such that $p^{*}(\phi)=0$. We are going to show that $\phi \in F^{k} K^{*}(X)$ for all $k \geq-1$. For $k \geq \operatorname{dim}(X)+1$ this then implies that $\phi=0$.

We clearly have $\phi \in F^{-1} K^{*}(X)=K^{*}(X)$. Assume now by induction that $\phi \in F^{k} K^{*}(X)$. Then $\phi$ is detected by an element $\sigma_{k}(\phi) \in E_{\infty}^{k, *}(\mathrm{id})$. We observe that the image of $\sigma_{k}(\phi)$ in $E_{\infty}^{k, *}(p)$ and in particular its component in $p^{*} E_{\infty}^{k, *}(\mathrm{id})$ vanishes. By Lemma 6.5 we have $\sigma_{k}(\phi)=0$. This implies $\phi \in F^{k+1} K^{*}(X)$.

Lemma 6.7 The range of the map 126 is dense.

Proof. We fix an element $\phi \in K^{*}(Y)$. We must show that we can approximate this element in the profinite topology of $K^{*}(Y)$ by elements in the image of $p^{*}: K^{*}(X) \rightarrow K^{*}(Y)$. Let $t: T \rightarrow Y$ be a map from a finite $C W$-complex. Then $\operatorname{ker}\left(t^{*}\right) \subseteq K^{*}(Y)$ is some neighbourhood of zero. We must find an element $\psi \in K^{*}(X)$ such that

$$
\phi-p^{*} \psi \in \operatorname{ker}\left(t^{*}\right)
$$

To this end we use the following Lemma

Lemma 6.8 If $\hat{\phi} \in F^{k} K^{*}(Y)$, then there exists $\hat{\psi} \in K^{*}(X)$ and $\rho \in \operatorname{ker}\left(t^{*}\right)$ such that $\hat{\phi}-p^{*} \hat{\psi}-\rho \in F^{k+1} K^{*}(Y)$.

Assuming this Lemma and using that $\phi \in F^{-1} K^{*}(Y)$ we obtain the desired $\psi \in K^{*}(X)$ by a finite iteration. Thus Lemma 6.7 follows from Lemma 6.8 .

Proof. [of Lemma 6.8 The element $\hat{\phi}$ gives rise to an element $u:=\sigma_{k}(\hat{\phi}) \in E_{\infty}^{k, *}(p)$ which we can decompose as $u=v \oplus p^{*} w$ with $v \in E_{\infty}^{k, *}(p)_{\text {div }}$ and $w \in E_{\infty}^{k, *}(\mathrm{id})$. We let $\hat{\psi} \in F^{k} K^{*}(X)$ be an element which is detected by $w$. We apply Lemma 6.3 to the exact sequence

$$
0 \rightarrow F^{k+1} K^{*}(Y) \rightarrow F^{k} K^{*}(Y) \rightarrow E_{\infty}^{k, *}(p) \rightarrow 0
$$

and the map $t^{*}: F^{k} K^{*}(Y) \rightarrow K^{*}(T)$. By Lemma 6.3 we can find an element $\rho \in$ $F^{k} K^{*}(Y) \cap \operatorname{ker}\left(t^{*}\right)$ which represents $v$. Then we have $\phi-p^{*} \hat{\psi}-\rho \in F^{k+1} K^{*}(Y)$ as required.

Proposition 6.4 now follows from Lemma 6.6 and Lemma 6.7.

Proof. [of Proposition 6.1]

Let

$$
\emptyset=Y_{-1} \subseteq Y_{0} \subseteq Y_{1} \subseteq Y_{2} \subseteq \ldots
$$

be the filtration of $Y$ induced by taking the preimages of the subcomplexes $X_{k}$ along $p: Y \rightarrow X$. By construction the map $p$ preserves filtrations and hence 124 is continuous. 
We first show that (124) is injective. Note that the filtration $\left(F^{k} K^{*}(X)\right)_{k \geq 0}$ induces the profinite topology. Let $\phi \in K^{*}(X)$ be such that $p^{*} \phi \in \bigcap_{k \geq 0} F^{k} K^{*}(Y)$. It suffices to show that this implies $\phi \in F^{k} K^{*}(X)$ for all $k \geq 0$ since then $\phi$ represents the zero element in $\bar{K}^{*}(X)$.

We clearly have $\phi \in F^{-1} K^{*}(X)$. We now assume by induction that $\phi \in F^{k} K^{*}(X)$. We can apply Proposition 6.4 to the fibration $p_{k}: Y_{k} \rightarrow X_{k}$. Since

$$
p_{k}^{*}\left(\phi_{\mid X_{k}}\right)=\left(p^{*} \phi\right)_{\mid Y_{k}}=0
$$

we conclude that $\phi_{\mid X_{k}}=0$ and hence $\phi \in F^{k+1} K^{*}(X)$.

We now show that the image of (125) is dense. Let $\phi \in K^{*}(Y)$. We must approximate $\phi$ by elements in the image of (125). Let $t: T \rightarrow Y$ be a map from a finite $C W$-complex. Then $\operatorname{ker}\left(t^{*}\right) \subseteq K^{*}(Y)$ is some neighbourhood of zero. We must find an element $\psi \in K^{*}(X)$ such that

$$
\phi-p^{*} \psi \in \operatorname{ker}\left(t^{*}\right) .
$$

Since $T$ is finite there exists a number $k \in \mathbb{N}$ such that there is a factorisation

$$
t: T \stackrel{t_{k}}{\rightarrow} Y_{k} \stackrel{\iota k}{\rightarrow} Y
$$

It suffices to find an element $\psi \in K^{*}(X)$ such that

$$
\iota_{k}^{*} \phi-\iota_{k}^{*} p^{*} \psi \in \operatorname{ker}\left(t_{k}^{*}\right) .
$$

Then indeed $\phi-p^{*} \psi \in \operatorname{ker}\left(t^{*}\right)$.

For every $r \in \mathbb{N}$ we apply Proposition 6.4 to the fibration $p_{k+r}: Y_{k+r} \rightarrow X_{k+r}$ in order to see that $p_{k+r}^{*}$ induces an isomorphism

$$
\frac{K^{*}\left(X_{k+r}\right)}{\operatorname{ker}\left(\left(p_{k+r} \circ t_{k+r}\right)^{*}\right)} \stackrel{\sim}{\rightarrow} \frac{K^{*}\left(Y_{k+r}\right)}{\operatorname{ker}\left(t_{k+r}^{*}\right)} .
$$

We have a projective system of exact sequences indexed by $r \in \mathbb{N}$

$$
0 \rightarrow \operatorname{ker}\left(\left(p_{k+r} \circ t_{k+r}\right)^{*}\right) \rightarrow K^{*}\left(X_{k+r}\right) \rightarrow \frac{K^{*}\left(Y_{k+r}\right)}{\operatorname{ker}\left(t_{k+r}^{*}\right)} \rightarrow 0
$$

If we take the limit, identify

$$
\frac{\lim _{r} K^{*}\left(X_{k+r}\right)}{\lim _{r} \operatorname{ker}\left(\left(p_{k+r} \circ t_{k+r}\right)^{*}\right)} \cong \frac{\bar{K}^{*}(X)}{\operatorname{ker}\left((p \circ t)^{*}\right)}
$$

and use the assumption that $\lim _{r}^{1} K^{*}\left(X_{k+r}\right)=0$, then we get the exact sequence

$$
0 \rightarrow \frac{\bar{K}^{*}(X)}{\operatorname{ker}\left((p \circ t)^{*}\right)} \rightarrow \lim _{r} \frac{K^{*}\left(Y_{k+r}\right)}{\operatorname{ker}\left(t_{k+r}^{*}\right)} \rightarrow \lim _{r}^{1} \operatorname{ker}\left(\left(p_{k+r} \circ t_{k+r}\right)^{*}\right) \rightarrow 0
$$


Note note that the graded groups

$$
\lim _{r} \frac{K^{*}\left(Y_{k+r}\right)}{\operatorname{ker}\left(t_{k+r}^{*}\right)} \cong \bigcap_{r \in \mathbb{N}} \operatorname{im}\left(t_{k+r}^{*}\right), \quad \operatorname{ker}\left(\left(p_{k+r} \circ t_{k+r}\right)^{*}\right)
$$

are of finite type, since they are subgroups of the groups of finite type $K^{*}(T)$ and $K^{*}\left(X_{k+r}\right)$, respectively.

Fact 6.9 ([31]) If $\left(G_{r}\right)_{r \in \mathbb{N}}$ is a projective system of countable abelian groups, then either $\lim _{r}^{1} G_{r}=0$ or $\lim _{r}^{1} G_{r}$ is uncountable.

Since $\lim _{r}^{1} \operatorname{ker}\left(\left(p_{k+r} \circ t_{k+r}\right)^{*}\right)$ is a quotient of a countable group we conclude that this group is actually trivial. The pull-back along the family of maps $\left(p \circ i_{k+r}\right)_{r \in \mathbb{N}}$ induces an isomorphism

$$
\frac{\bar{K}^{*}(X)}{\operatorname{ker}\left((p \circ t)^{*}\right)} \stackrel{\sim}{\rightarrow} \lim _{r} \frac{K^{*}\left(Y_{k+r}\right)}{\operatorname{ker}\left(t_{k+r}^{*}\right)} .
$$

The element $\phi$ represents a class $[\phi] \in \lim _{r} K^{*}\left(Y_{k+r}\right) / \operatorname{ker}\left(t_{k+r}^{*}\right)$. Hence we can find a class $[\psi] \in \bar{K}^{*}(X) / \operatorname{ker}\left((p \circ t)^{*}\right)$ which is mapped to $[\phi]$ under the isomorphism above. The elements $\phi$ and $\psi$ satisfy the relation $(129)$. This finishes the proof of Proposition 6.1.

\section{References}

[1] J. F. Adams. On the groups $J(X)$. IV. Topology, 5:21-71, 1966.

[2] J. F. Adams. Infinite loop spaces, volume 90 of Annals of Mathematics Studies. Princeton University Press, Princeton, N.J., 1978.

[3] J. F. Adams. Stable homotopy and generalised homology. Chicago Lectures in Mathematics. University of Chicago Press, Chicago, IL, 1995. Reprint of the 1974 original.

[4] D. W. Anderson and L. Hodgkin. The $K$-theory of Eilenberg-MacLane complexes. Topology, 7:317-329, 1968.

[5] M. F. Atiyah, V. K. Patodi, and I. M. Singer. Spectral asymmetry and Riemannian geometry. I. Math. Proc. Cambridge Philos. Soc., 77:43-69, 1975.

[6] M. F. Atiyah, V. K. Patodi, and I. M. Singer. Spectral asymmetry and Riemannian geometry. II. Math. Proc. Cambridge Philos. Soc., 78(3):405-432, 1975.

[7] M. F. Atiyah, V. K. Patodi, and I. M. Singer. Spectral asymmetry and Riemannian geometry. III. Math. Proc. Cambridge Philos. Soc., 79(1):71-99, 1976.

[8] M. F. Atiyah and G. B. Segal. Equivariant K-theory and completion. J. Differential Geometry, 3:1-18, 1969. 
[9] M. F. Atiyah and I. M. Singer. The index of elliptic operators. I. Ann. of Math. (2), 87:484-530, 1968.

[10] A. Bahri and P. Gilkey. The eta invariant, $\operatorname{Pin}^{c}$ bordism, and equivariant $\operatorname{Spin}^{c}$ bordism for cyclic 2-groups. Pacific J. Math., 128(1):1-24, 1987.

[11] A. Bahri and P. Gilkey. $\operatorname{Pin}^{c}$ cobordism and equivariant $\operatorname{Spin}^{c}$ cobordism of cyclic 2-groups. Proc. Amer. Math. Soc., 99(2):380-382, 1987.

[12] T. Bauer. Computation of the homotopy of the spectrum tmf. In Groups, homotopy and configuration spaces, volume 13 of Geom. Topol. Monogr., pages 11-40. Geom. Topol. Publ., Coventry, 2008.

[13] P. Baum and R. G. Douglas. K homology and index theory. In Operator algebras and applications, Part I (Kingston, Ont., 1980), volume 38 of Proc. Sympos. Pure Math., pages 117-173. Amer. Math. Soc., Providence, R.I., 1982.

[14] N. Berline, E. Getzler, and M. Vergne. Heat kernels and Dirac operators. Grundlehren Text Editions. Springer-Verlag, Berlin, 2004. Corrected reprint of the 1992 original.

[15] B. Blackadar. K-theory for operator algebras, volume 5 of Mathematical Sciences Research Institute Publications. Cambridge University Press, Cambridge, second edition, 1998.

[16] J. M. Boardman. Stable operations in generalized cohomology. In Handbook of algebraic topology, pages 585-686. North-Holland, Amsterdam, 1995.

[17] A. K. Bousfield. The localization of spectra with respect to homology. Topology, 18(4):257-281, 1979.

[18] J.-L. Brylinski. Loop spaces, characteristic classes and geometric quantization. Modern Birkhäuser Classics. Birkhäuser Boston Inc., Boston, MA, 2008. Reprint of the 1993 edition.

[19] U. Bunke. A $K$-theoretic relative index theorem and Callias-type Dirac operators. Math. Ann., 303(2):241-279, 1995.

[20] U. Bunke. Index theory, eta forms, and Deligne cohomology. Mem. Amer. Math. Soc., 198(928):vi+120, 2009.

[21] U. Bunke and N. Naumann. Secondary Invariants for String Bordism and tmf. ArXiv e-prints, December 2009.

[22] U. Bunke and Th. Schick. Smooth K-theory. Astérisque, (328):45-135 (2010), 2009.

[23] U. Bunke and Th. Schick. Uniqueness of smooth extensions of generalized cohomology theories. J. Topol., 3(1):110-156, 2010. 
[24] D. Crowley and S. Goette. Kreck-Stolz invariants for quaternionic line bundles. ArXiv e-prints, December 2010.

[25] P. Deligne. La formule de dualite globale, volume Theorie des topos et cohomologie etale des schemas. Tome 3 of Lecture Notes in Mathematics 305. Springer-Verlag, Berlin, 1973.

[26] Ch. Deninger and W. Singhof. The e-invariant and the spectrum of the Laplacian for compact nilmanifolds covered by Heisenberg groups. Invent. Math., 78(1):101-112, 1984.

[27] H. Donnelly. Spectral geometry and invariants from differential topology. Bull. London Math. Soc., 7:147-150, 1975.

[28] A. Z. Dymov. Homology spheres and contractible compact manifolds. Izv. Akad. Nauk SSSR Ser. Mat., 35:72-77, 1971.

[29] D. S. Freed and M.J. Hopkins. On Ramond-Ramond fields and $K$-theory. J. High Energy Phys., (5):Paper 44, 14, 2000.

[30] D. S. Freed and R. B. Melrose. A mod $k$ index theorem. Invent. Math., 107(2):283299, 1992.

[31] B. I. Gray. Spaces of the same $n$-type for all $n$. Topology, 5:241-243, 1966.

[32] F. Hirzebruch, Th. Berger, and R. Jung. Manifolds and modular forms. Aspects of Mathematics, E20. Friedr. Vieweg \& Sohn, Braunschweig, 1992. With appendices by Nils-Peter Skoruppa and by Paul Baum.

[33] N. Hitchin. Lectures on special Lagrangian submanifolds. In Winter School on Mirror Symmetry, Vector Bundles and Lagrangian Submanifolds (Cambridge, MA, 1999), volume 23 of AMS/IP Stud. Adv. Math., pages 151-182. Amer. Math. Soc., Providence, RI, 2001.

[34] M. J. Hopkins. Algebraic topology and modular forms. In Proceedings of the International Congress of Mathematicians, Vol. I (Beijing, 2002), pages 291-317, Beijing, 2002. Higher Ed. Press.

[35] M. J. Hopkins and I. M. Singer. Quadratic functions in geometry, topology, and M-theory. J. Differential Geom., 70(3):329-452, 2005.

[36] M. A. Hovey. $v_{n}$-elements in ring spectra and applications to bordism theory. Duke Math. J., 88(2):327-356, 1997.

[37] M. A. Hovey. The homotopy of $M$ String and $M \mathrm{U}\langle 6\rangle$ at large primes. Algebr. Geom. Topol., 8(4):2401-2414, 2008. 
[38] M. A. Hovey and D. C. Ravenel. The 7-connected cobordism ring at $p=3$. Trans. Amer. Math. Soc., 347(9):3473-3502, 1995.

[39] J. D. S. Jones and B. W. Westbury. Algebraic $K$-theory, homology spheres, and the $\eta$-invariant. Topology, 34(4):929-957, 1995.

[40] G. G. Kasparov. Equivariant $K K$-theory and the Novikov conjecture. Invent. Math., 91(1):147-201, 1988.

[41] Michel A. Kervaire and John W. Milnor. Groups of homotopy spheres. I. Ann. of Math. (2), 77:504-537, 1963.

[42] M. Kreck and St. Stolz. A diffeomorphism classification of 7-dimensional homogeneous Einstein manifolds with $\mathrm{SU}(3) \times \mathrm{SU}(2) \times \mathrm{U}(1)$-symmetry. Ann. of Math. (2), 127(2):373-388, 1988.

[43] H. B. Lawson, Jr. and M.-L. Michelsohn. Spin geometry, volume 38 of Princeton Mathematical Series. Princeton University Press, Princeton, NJ, 1989.

[44] J. Lurie. A survey of elliptic cohomology. In Algebraic topology, volume 4 of Abel Symp., pages 219-277. Springer, Berlin, 2009.

[45] E. Y. Miller and R. Lee. Some invariants of spin manifolds. Topology Appl., 25(3):301311, 1987.

[46] M. K. Murray. An introduction to bundle gerbes. In The many facets of geometry, pages 237-260. Oxford Univ. Press, Oxford, 2010.

[47] D. Quillen. Letter from Quillen to Milnor on $\operatorname{Im}\left(\pi_{i} 0 \rightarrow \pi_{i}^{\mathrm{s}} \rightarrow K_{i} \mathbf{Z}\right)$. In Algebraic K-theory (Proc. Conf., Northwestern Univ., Evanston, Ill., 1976), pages 182-188. Lecture Notes in Math., Vol. 551. Springer, Berlin, 1976.

[48] D. C. Ravenel. Complex cobordism and stable homotopy groups of spheres, volume 121 of Pure and Applied Mathematics. Academic Press Inc., Orlando, FL, 1986.

[49] Y. B. Rudyak. On Thom spectra, orientability, and cobordism. Springer Monographs in Mathematics. Springer-Verlag, Berlin, 1998. With a foreword by Haynes Miller.

[50] J. A. Seade. On the $\eta$-function of the Dirac operator on $\Gamma \backslash S^{3}$. An. Inst. Mat. Univ. Nac. Autónoma México, 21(1):129-147 (1982), 1981.

[51] J.-P. Serre. Groupes d'homotopie et classes de groupes abéliens. Ann. of Math. (2), 58:258-294, 1953.

[52] A. A. Suslin. On the $K$-theory of local fields. In Proceedings of the Luminy conference on algebraic K-theory (Luminy, 1983), volume 34, pages 301-318, 1984.

[53] K. Waldorf. String connections and Chern-Simons theory. Trans. Amer. Math. Soc., 365(8):4393-4432, 2013. 